SLAC -292

\section{DE86 009666}

SLAC -292

$\mathrm{UC}-34 \mathrm{D}$

(E)

\section{DECAYS OF THE TAU LEPTON *}

Patricia R. Burchat

Stanford Linear Accelerator Center

Stanford University

Stanford, California 94305

Printed in the United States of America. Available from the National Technical Information Service, U.S. Department of Commerce, 5285 Port Royal Road,
February 1986

Prepared for the Department of Energy ander contract number DE-ACO3-76SFO0515

Springfield, Virginia 22161. Price: Printed Copy A07, Microfiche A01.

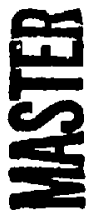

\section{Abstract}

Previous measurements of the branching fractions of the tau lepton result in a discrepancy between the inclusive branching fraction and the sum of the exclusive branching fractions to final states containing one charged particle. The sum of the exclusive branching fractions is significantly smaller than the inclusive branching fraction. In this analysis, the branching fractions for all the major decay modes are measured simr ltaneously with the sum of the branching fractions constrained to be one. The branching fractions are measured using an unbiased sample of tau decays, with little background, selected from $207 \mathrm{pb}^{-1}$ of data accumulated with the Mark II detector at the PEP $e^{+} e^{-}$storage ring. The sample is selected using the decay products of one member of the $\tau^{+} \tau^{-}$pair produced in $e^{+} e^{-}$annihilation to identify the event and then including the opposite member of the pair in the sample. The sample is divided into subgroups cecording to charged and neutral particle multiplicity, and charged particle identi ication. The branching fractions are simultaneously measured using an unfold echnique and a maximum likelihood fit.

The results of this analysis indicate that the discrepancy found in previous experiments is possibly due to two sources. First, the leptonic branching fractions measured is this analysis are about one atandard deviation higher than the world average, resulting in a total exr $\quad(2.0 \pm 1.8) \%$ over the world average in these two modes. The me ed leptonic branching fractions correspond to a tau lifetime of $(1) \perp 0.2) \times 10^{-13} \mathrm{~s}$ which is slightly longer than the average measured tau lifetime of $(2.8 \pm 0.2) \times 10^{-13} \mathrm{~s}$. Secondly, the total branching fraction to one charged hadron plus at least one neutral particle is masured to be $(7 \pm 3) \%$ higher than the branching fraction expected from a combination of previous measurements and theoretical predictions. It is shown that decay modes involving the $\eta$ are not expected to contribute more than $3 \%$ to this excess. 
I wish to thank my advisor, Gary Feldman, for suggesting an interesting and challenging thesis topic; John Yelton, for providing patient guidance in learning analysis techniques; Mark Neison, for invaluable advise, well documented programs, and a very useful thesis; and Ken Hayes, for friendly discussions and advise.

I am indebted to Gajl Hanson, Hartrnut Sadrozinski, Dave Hutchinson and $\mathrm{A}$ be Seiden for the enjoyable experience of working with them and learning from them during uny early days at SLAC.

With pleasure, I extend a warm thanks to those friends who made my graduatc student days especially memorable: Barb and Theo Schaad, for their company and appetite for something different; all my friends at Campo Rello, especially Mary James and Alain Schwartzman, for sharing a warm and friendly home: Bill Rowe, for many laughs; and Alan Weinstein, for providing an example of how to combine hard work and hard play.

I wish to thank my family in Canada for providing such a warn welcome earh time 1 go home. Finally, I wish to thank Tony Norcia, who has added to the pleasure of my life both at home and at work, for his encouragement and support in seeing me through this thesis.

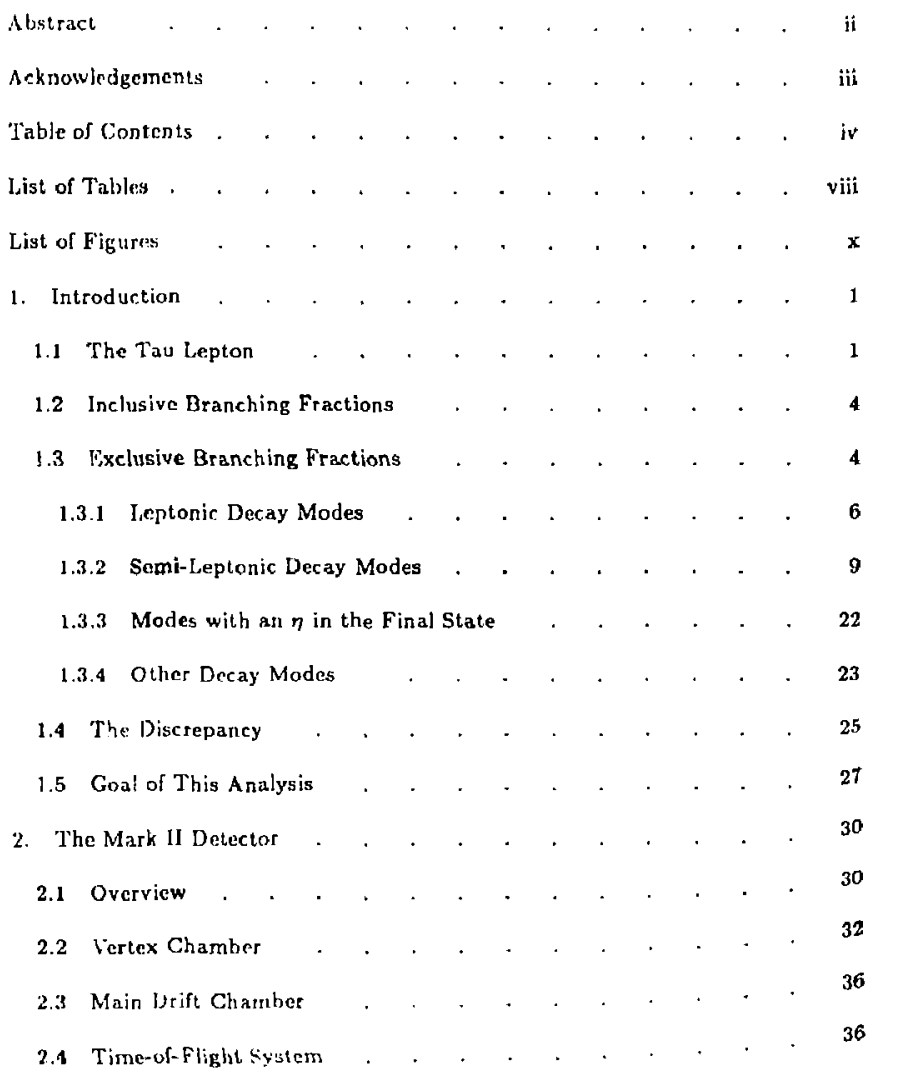


2.5 Solenoidal Magnet . . . . . . . . . . . . 36

2.6 Lead - Liquid Argon Electromagnetic Calorimeter . . . . 38

2.7 Muon Identification System . . . . . . . . . 39

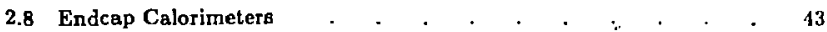

2.9 Small Angle Calorimeter $\quad$. . . . . . . . . . . 43

2.10 Event Trigger . . . . . . . . . . . 43

3. Particle Identification . . . . . . . . . . . . . . 46

3.1 Muon Identification . . . . . . . . . . . 47

3.1.1 $\mu / \pi$ Discrimination . . . . . . . . . . 48

3.1.2 Muon Rejection . . . . . . . . . . 49

3.2 Electron Identification . . . . . . . . . . . 50

3.2.1 $e / \pi$ Discrimination . . . . . . . . . . 51

3.2.2 Electron Rejection . . . . . . . . . . 53

3.3 Photon Identification . . . . . . . . . . 57

$3.4 e^{+} e^{- \text {Pair-Finding Algorithr. }} . \quad . \quad . \quad . \quad . \quad . \quad 58$

4. Event Selection . . . . . . . . . . . . 62

4.1 General Philosophy _. . . . . . . . . . . 62

4.2 Overall Cuts . . . . . . . . . . . . 63

4.3 The 1 Prong Tag . . . . . . . . . . . . 64

4.3.1 Selection Criteria $\quad . \quad . \quad . \quad . \quad . \quad . \quad . \quad . \quad . \quad .64$

4.3.2 Efficiency . . . . . . . . . . . . 66
4.4 The 3 Prong Tag . . . . . . . . . . . 67

4.4.1 Selection Criteria . . . . . . . . . . 67

4.4.2 Efficiency . . . . . . . . . . . . . . 69

5. Unfolding the Branching Fractions . . . . . . . . 71

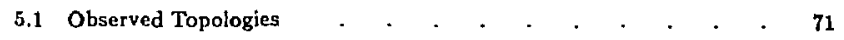

5.2 The Unfold Technique . . . . . . . . . . 74

5.3 The Efficiency Matrix + . . . . . . . . . 76

5.4 Monte Carlo Simulation . . . . . . . . . 80

5.5 Particle Identification Efficiency . . . . . . . . . . 82

5.5.1 Muon Identification $\quad$. . . . . . . . . . . 82

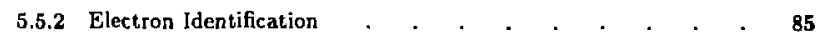

5.5.3 Pion Identification . . . . . . . . . . 87

5.6 Angular Distribution . . . . . . . . . . 90

5.7 Momentum Distribution . . . . . . . . . . 93

6. Backgrounds . . . . . . . . . . . . . 96

6.1 Background from Two Photon Production of Tau Pairs . . . 96

6.2 Background from Two Photon Production of $q-\bar{q}$. . . . . 98

6.3 Backgrounds to the 1 Prong Tag . . . . . . . . . . 98

6.3.1 Leptonic Backgrounds . . . . . . . . 98

6.3.2 Muon Rejection Efficiency . . . . . . . . . . 99

6.3.3 Electron Rejection Efficiency _. . . . . . . . 100 
6.3.4 Background from Single Photon Production of Lepton Pairs · 101

6.3.5 Background from Two Photon Production of Lepton Pairs . 101

6.3.6 Background from Single Photon Production of $q-\bar{q}$. . 103

6.4 Background to the 3 Prong Tag . . . . . . . . 104

6.4.1 Background from Single or Two Photon Production of Lepton Pairs 104

6.4.2 Background from Single Photon Production of $q-\bar{q}$. . 105

6.5 Summary of Backgrounds . . . . . . . . . . . . 106

7. Results . . . . . . . . . . . . . . 111

7.1 Measured Branching Fractions . . . . . . . . 111

7.2 Systematic Errors . . . . . . . . . . . 113

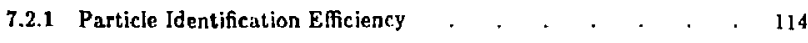

7.2.2 Relative Overall Efficiency . . . . . . . . . 116

7.2.3 Background Estimates $\quad$. . . . . . . . . 117

7.2.4 Summary of Systematic Errors . . . . . . . 117

7.3 Results . . . . . . . . . . . . . 117

7.3.1 Limits on Decay Modes Involving the $\eta \quad$. . . . . . 121

7.4 Conclusions . . . . . . . . . . . . . . 122

Appendix A. Selection of Known Leptons in the Data . . . . 123

REFERENCES . . . . . . . . . . . . . 126

\section{List of Tables}

1.1 The known lepton families . . . . . . . . . 2

1.2 Inclusive branching fractions . . . . . . . . 5

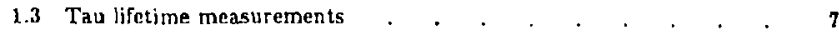

1.4 Published measurements of $B\left(r^{-} \rightarrow \nu_{\tau} e^{-} \nu_{\varepsilon}\right)$. . . . . . . 8

1.5 Published measurementz of $B\left(\tau^{-} \rightarrow \nu_{\tau} \mu^{-} V_{\mu}\right)$. . . . . . 9

1.6 Quantum numbers of the weak charged current . . . . 10

1.7 Published measurenents of $B\left(\tau^{-} \rightarrow \nu_{\mathrm{r}} \pi^{-}\right) \quad$. . . . . . . 13

1.8 Published measurements of $B\left(r^{-} \rightarrow \nu_{r} \rho^{-}\right) \quad$. . . . . . 14

1.9 Published measurements of $B\left(\tau^{-} \rightarrow \nu_{\tau} \pi^{-} \pi^{+} \pi^{-}\right)$. . . . 15

1.10 Published measurements of $B\left(\tau^{-} \rightarrow \nu_{r} \pi^{-} \pi^{+} \pi^{-} \pi^{0}\right)$. . . 17

1.11 Published measurements of $B\left(\tau^{-} \rightarrow \nu_{r} K^{-}\right)$. . . . . 21

1.12 I'ublished measurements of $B\left(\tau^{-} \rightarrow \nu_{r} K^{*-}\right)$. . . . . . 21

1.13 Decay modes involving an $\eta \quad . \quad, \ldots, \ldots, \ldots$

1.14 Branching fractions for all decay modes . . . . . . 26

1.15 Published measurements of $\sigma\left(e^{+} e^{-} \rightarrow \tau^{+} \tau^{-}(\gamma)\right)$. . . 27

2.1 Radii of sense wire Jayers in vertex chamber . . . . . 34

2.2 Hadron absorber thickness in muon system . . . . . 41

3.1 Extrapolation error for muon system . . . . . . . 48

3.2 Longitudinal shower development . . . . . . , 52

3.3 Shower width , . . . . . . . . , . 53

5.1 Criteria for classification of a single charged particle , . , 72

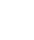

$\sqrt{2}$
3

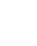

.

7

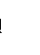

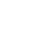


5.2 Topological distribution of sample $\quad . \quad$. . . . . . 74

5.3 Efficiency matrix for the 1 prong tag . . . . . . . 78

5.4 Efficiency matrix for the 3 prong tag . . . . . . . 79

6.1 Background from $e^{+} e^{-} \rightarrow e^{+} e^{-} \tau^{+} \tau^{-} \quad . \quad . \quad . \quad . \quad .97$

6.2 Background from single photon production of lepton pairs . . 102

6.3 Background from two photon production of lepton pairs _ . 102

6.4 Backgrounds in 1 prong tag . . . . . . . . . . . 107

6.5 Backgrounds in 3 prong tag . . . . . . . . . . . 108

6.3 Background subtracted sample for 1 prong tag _ . . . . . 109

6.7 Background subtracted sample for 3 prong tag _ . . . 110

7.1 Topological distribution for best fit . . . . . . . . 112

7.2 Measured branching fractions , . . . . . . . 113

7.3 Currelation coefficienta for 1 prong tag . . . . . . 113

7.4 Correlation coefficients for the 3 prong tag . . . . . 114

7.5 Summary of systematic errors _ . . . . . . . . . 118

7.6 Measured branching fractions . . . . . . . . 119

\section{DISCLAIMER}

This report was prepared as an account of work sponsored by an agency of the United States Government. Neither the United States Government nor any agency therenf, nor any of their
employees, makes any warranty, express or implied, or assumes any legal liability or responsiemployees, makes any warranty, express or implied, or assumes any legal liability or responsibility for the accuracy, completeness, or usefulness of any information, apparalus, producl, or process disclosed, or represents that its use would not infringe privately owned rights. Reference herein to any specific commercial product, process, or service by trade name, tradcmark. manufactures, or otherwise does not necessarily constitute or imply its endorsement, recommendation, or favoring by the United States Government or any agency the and opinions of authors expressed herein do mat necessarily state or reflect those of the
United States Government or any agency thereof.

\section{List of Figures}

1.1 Feynman diagrams for the decay of the $\mu$ and $\tau$ leptons . . . 3

1.2 Feynman diagrams for $\tau^{-} \rightarrow \nu_{\tau} \pi^{-}$and $\pi^{-} \rightarrow \mu^{-} \sigma_{\mu}$. . . . 12

1.3 Feynman diagrames for $\tau^{-} \rightarrow \nu_{\tau} K^{-}$and $K^{-} \rightarrow \mu^{-} \nu_{\mu} . \quad . \quad . \quad 20$

2.1 The Mark II detector at PEP . . . . . . . 31

2.2 The Mark II vertex drift chamber . . . . . . . 33

2.3 Wire array for vertex detector . . . . . . . . . 35

2.4 Wire array for main drift chamber . . . . . . . . . 37

2.5 Ganging schenie in the LA calorimeter modules . . . . 40

2.6 Cross sectional view of a muon proportional tube module . . 42

3.1 TEST1 distribution for electrons and pions _ . . . . . 54

3.2 LA calorimeter efficiency $\quad$. . . . . . . . . . 56

3.3 Energy spectrum of photon candidales in Monte Carlo . . . 59

3.4 Parameters used in pair-finding algorithm . . . . . . 61

51 Muon identification efficiency . . . . . . . . 84

5.2 Electron identification efficiency . . . . . . . . . . . 86

$5.3 e / \pi$ discrimination for pions . . . . . . . . . . 89

5.4 Angular distribution of the 1 prong tag . . . . . . . 91

5.5 Angular distribution of the 3 prong tag . . . . . . 92

5.6 Momentum distribution of tagged one prongs, 1 prong tag . . 94

5.7 $M$ mentum distribution of tagged one prongs, 3 prong tag . . 95

A.I Momentum distribution of knowr kntons in the data . . . 125 
1. Introduction

\section{I.1 THE TAU LEPTON}

With the discovery of the tau lepton $1,2,3$ in 1975 at the SPEAR $e^{+} e^{-}$storage ring, the number of known families of leptons increased to three: the electron family $\left(e^{-}, \nu_{e}\right)$, the muon family $\left(\mu^{-}, \nu_{\mu}\right)$, and the tau family $\left(r^{-}, \nu_{r}\right)$. The mass and lifetime of each of these particles is listed in Table 1.1. The electron is stable while the muon and the tau decay via the weak interaction as shown in Figure 1.1. In the decay of both the muon and the tau, a virtual charged intermediate vector boson $\left(W^{ \pm}\right)$materializes into fermion-antifermion pairs. Because the mass of the muon is less than that of the lightest meson (the pion), the muon cannot decay to final states containing hadrons. The mass of the tau, on the other hand, is more than twelve times as great as the mass of the pion. Therefore, in tau decay the $W^{ \pm}$disintegrates into quark-antiquark pairs as well as leptons. Predictions of the coupling of the $W$ to quarks and leptons are tested by measuring the branching fractions of the tav to semi-leptonic or purely leptonic final states.

The production and decay of the tau lepton has been extensively studied at $\mathrm{e}^{+} \mathrm{e}^{-}$storage rings. Mcasurements of the total cross section, differential cross section, lifetime, and ratios of branching fractions agree well with the standard model. However, the sum of experimentally measured exclusive branching fractions to final states containing one charged particle is significantly smaller than the measured inclusive branching fraction to final states containing one charged particle. Either the measured branching fractions are incorrect or there exist significant decry
Table 1.1. The known lepton families.

\begin{tabular}{|c|c|c|c|}
\hline $\begin{array}{c}\text { family } \\
\text { лame }\end{array}$ & symbol & $\begin{array}{c}\text { mass } \\
\left(\mathrm{MeV} / \mathrm{c}^{2}\right)\end{array}$ & $\begin{array}{c}\text { lifetime } \\
\text { (seconds) }\end{array}$ \\
\hline electron & $e^{-}$ & 0.511 & stable \\
& $\nu_{e}$ & $<46 \mathrm{eV}$ & stable \\
\hline muon & $\mu^{-}$ & 105.7 & $2.2 \times 10^{-6}$ \\
& $\nu_{\mu}$ & $<0.5$ & stable \\
\hline tau & $r^{-}$ & 1784 & $2.8 \times 10^{-13}$ \\
& $\nu_{\mathrm{r}}$ & $<70$ & stable \\
\hline
\end{tabular}

modes for which the exclusive branching fraction has not been measured.

In the next section, I discuss published measurements of inclusive branching fractions of the tau to one, three and five charged particles. I then review theoretical predictions for and published experimental measurements of branching fractions to exclusive final states. Only measurements from experiments at the PEP and PETRA storage rings, and from the Mask II and Mark IIl experimes is at SPEAR, are reviewed. Results from early expcriments at SPEAR are not included in this review since some of them are in clear disagreernent with more precise measurements made by recent experiments.

I chen discuss the discrepancy which exists between the sum of exculsive branching fractions and the inclusive branching fraction to one-charged-particle final states. In the final section, I outline the philosophy of this analysis and the method which is used to solve the discrepancy mer :ioned above. 
1.2 InClusive Branching Fractions

Since the tau lepton has unit charge it decays to an odd number of charged particles. The branching fractions of the tau to one, three and five charged particles have been measured in many experiments and the published results are listed in Table 1.2. The average over all the experiments is also listed for each branching fraction. * The world average for the inclusive branchisg fraction to one charged particle, $B 1$, is $(86.8 \pm 0.3) \%$. Most of the remaining decays result in three charged particles. The recent precisc measurements of the HRS and MAC experiments contribute most heavily to these weighted means. The branching fraction to final states with five or more charged particles is very small and has only recently been measured to be non-zero.

In all measurements of the inclusive branching fractions, a sample of tau-pair events is selected based on criteria other than charged particle r' ultiplicity. Then the produced multiplicity distribution is deternined from the obs, -ved distribution by an unfold method. This method guarantees that $B 1+B 3+335=1$

\subsection{Exclu5ne Branching Fractions}

As mentioned above, the tau decays weakly to leptonic or semi-leptonic final 2.80
$5290 A 9$$\overline{\nu_{e}} \cdot \overline{\nu_{\mu}} \cdot \overline{\mathrm{U}}$ states. According to the standard model of weak interactions, there are five diagrams for the decay of the $r^{-}$which contribute approximi.cely equally: two

- To calculate the average, each measurement is weighted sy the inverse of its squared error where the error is the quadratic sum of the stalistical and systematic

Figure 1.1. Feynman diagrams for the decay of the muon and taulepton. 
Table 1.2. Inclusive branching fractions to one, three, and five-charged particles. For each measurement, the first error is statistical and the second is systematic.

\begin{tabular}{|c|c|c|c|}
\hline Experiment & $\mathrm{B1}$ & B3 & B5 \\
\hline Mark II 4,5 & $(86 \pm 2 \pm 1) \%$ & $(14 \pm 2 \pm 1) \%$ & $(0.16 \pm 0.08 \pm 0.04) \%$ \\
\hline CELI,O 6 & $(84.0 \pm 2.0) \%$ & $(15.0 \pm 2.0) \%$ & $(1.0 \pm 0.4) \%$ \\
\hline CELLO $^{7}$ & $(85.2 \pm 1.9 \pm 1.3) \%$ & $(14.8 \pm 1.5 \pm 1.3) \%$ & $<1.0 \%$ \\
\hline PLUTO ${ }^{8}$ & $(87.8 \pm 1.3 \pm 3.9) \%$ & $(12.2 \pm 1.3 \pm 3.9) \%$ & \\
\hline TA5SO ${ }^{9}$ & $\left(84.7 \pm 1.1_{-1.3}^{+1.6}\right) \%$ & $\left(15.3 \pm 1.1_{-1.6}^{+1.3}\right) \%$ & $<0.7 \%$ \\
\hline $\mathrm{TPC}^{10}$ & $(85.2 \pm 0.9 \pm 1.5) \%$ & $(14.8 \pm 0.9 \pm 1.5) \%$ & $<0.3 \%$ \\
\hline $\mathrm{MAC}^{11}$ & $(86.7 \pm 0.3 \pm 0.6) \%$ & $(13.3 \pm 0.3 \pm 0.6) \%$ & $<0.17 \%$ \\
\hline HRS 12,13 & $(86.9 \pm 0.2 \pm 0.3) \%$ & $(13.0 \pm 0.2 \pm 0.3) \%$ & $(0.13 \pm 0.04) \%$ \\
\hline $\mathrm{JADE}{ }^{14}$ & $(86.1 \pm 0.5 \pm 0.9) \%$ & $(13.6 \pm 0.5 \pm 0.8) \%$ & $(0.3 \pm 0.1 \pm 0.2) \%$ \\
\hline DELCO ${ }^{15}$ & $(87.9 \pm 0.5 \pm 1.2) \%$ & $(12.1 \pm 0.5 \neq 1.2) \%$ & \\
\hline Average & $(86.8 \pm 0.3) \%$ & $(13.1 \pm 0.3) \%$ & $(0.14 \pm 0.04) \%$ \\
\hline
\end{tabular}

in which the $W^{-}$couples to a lepton and its corresponding neutrino $\left(e^{-} \nu_{e}\right.$ and $\left.\mu^{-} D_{\mu}\right)$, and three in which the $W^{-}$couples to a quar ${ }^{-}$-antiquark pair $\left(\bar{u} d_{\theta}\right)$ in each of the three different colors. Therefore, the branching fractions for the leptonic decays $\tau^{-} \rightarrow \nu_{\tau} e^{-} \nu_{e}$ and $\tau^{-} \rightarrow \nu_{r} \mu^{-} \nu_{\mu}$ are expected to be approximately $20 \%$ each. Semi-leptonic decays of the tau are expected to occur approximately $60 \%$ of the time. Experimentally measured branching frartions agree approximately with these crude predictions.

More precise theoretical calculations generally predict the partial width for a particular decay mode of the tau lepton and not the absolute magnitude of the branching fraction for that decay mode. The theoretical predictions for the ratios of decay widths agree well with the experimentally measured ratios of branching fractions. The branching fraction can be calculated from the partial width if the total decay width is known. Since the total decay width is inversely proportional to the lifetime, experimental measurements of the tau lifetime, combined with theoretical predictions of the partial decay widths, lead to predictions of the branching fractions.

In the following sections, I revicw the theoretical predictions for decay widths by Y. S. Tsai, ${ }^{16}$ and by F. J. Gilman and Sun Hong Rhie. ${ }^{17}$ I also review the status of published measurements of branching fractions for each decay mode and compare these values with the theoretical predictions.

\subsubsection{Leptonic Deca: Modes}

\section{$\tau^{-} \rightarrow \nu_{T} e^{-} D_{e}$}

The decay width for $\tau^{-} \rightarrow \nu_{\gamma} e^{-} \bar{\nu}_{e}$, neglecting the mass of the electron, is given

by

$$
\Gamma\left(\tau^{-} \rightarrow \nu_{\tau} e^{-} \nabla_{e}\right)=\frac{G_{F}^{2} m_{\tau}^{5}}{192 \pi^{3}}
$$

assuming the standard madel with $V-A$ coupling of universal strength at the $\tau-\nu_{\tau}-W$ vertex. The decay width for $\tau^{-} \rightarrow \nu_{\tau} e^{-} \bar{\nu}_{e}$ can also be related to the tau lifetime through the branching ratio $B\left(\tau^{-} \rightarrow \nu_{r} e^{-} \nu_{e}\right)$ :

$$
B\left(\tau^{-} \rightarrow \nu_{\tau} e^{-} \nu_{e}\right)=\tau_{\tau} \times \Gamma\left(\tau^{-} \rightarrow \nu_{\tau} e^{-} \nu_{e}\right)
$$


The published measurements of the tau lifetime are listed in Table 1.3. Although the early measurements do not contribute significantly to the weighted average, it. is interesting to note the convergence to the present value from early longer lifetimes. Using the average mieasured lifetime of $(2.80 \pm 0.20) \times 10^{-13} s$ and a tau mass of $1784 \mathrm{MeV} / \mathrm{c}^{2}$, a combination of the above two equations results in $B\left(\tau^{-} \rightarrow \nu_{r} e^{-} \nu_{e}\right)=(17.6 \pm 1.3) \%$.

Table 1.3. Published measurements of the tau lifetime. Where two errors are listed, the first error is statistical and the second is systematic.

\begin{tabular}{|c|c|}
\hline Experiment & lifetime $(s)$ \\
\hline Mark I1 ${ }^{18}$ & $(1.6 \pm 1.9) \times 10^{-13}$ \\
CELLO $^{10}$ & $\left(4.7_{-2.9}^{+3.9}\right) \times 10^{-13}$ \\
Mark I1 $^{20}$ & $(2.86 \pm 0.16 \pm 0.25) \times 10^{-13}$ \\
TASSO $^{21}$ & $\left(3.18_{-0.75}^{+0.59} \pm 0.56\right) \times 10^{-13}$ \\
MAC $^{22}$ & $(2.67 \pm 0.21 \pm 0.22) \times 10^{13}$ \\
HRS $^{23}$ & $(2.8 \pm 0.4 \pm 0.5) \times 10^{-13}$ \\
\hline Average & $(2.80 \pm 0.20) \times 10^{-13}$ \\
\hline
\end{tabular}

Published measurements of $B\left(\tau^{-} \rightarrow \nu_{\tau} e^{-\cdots} V_{c}\right)$ are listed in Table 1.4 along with the event topologies used in each measurement. The average is dominated by the recent precise measurements by the MAC and Mark III experirnents. The average measured value for $B\left(\tau^{-} \rightarrow \nu_{\tau} e^{-} \nu_{e}\right)$ and the mensured tau lifetime agree very well with theory.

Table 1.4. Published measurements of $B\left(\tau^{-} \rightarrow \nu_{r} e^{-} L_{c}\right)$.

\begin{tabular}{|c|c|c|}
\hline Experiment & Branching Fraction & Method \\
\hline Mark II ${ }^{24}$ & $(17.5 \pm 0.6 \pm 1.0) \%$ & $e-\mu$ final states, \\
& & $\begin{array}{c}\text { dssumes } \varepsilon-\mu \text { universality } \\
\end{array}$ \\
PLUTO $^{8}$ & $(13.0 \pm 1.9 \pm 2.9) \%$ & final states, $X \neq e$ \\
TASSO $^{8}$ & $\left(20.4 \pm 3.0_{-0.9}^{+1.4}\right) \%$ & $e-X$ final states, $X \neq e$ \\
CELLO $^{25}$ & $(18.3 \pm 2.4 \pm 1.9) \%$ & $e-X$ final states, $X \neq e$ \\
Mark III ${ }^{26}$ & $(18.2 \pm 0.7 \pm 0.5) \%$ & $e-\mu_{,} e-e$ and $\mu-\mu$ final states \\
MAC 27 & $(17.8 \pm 0.5) \%$ & $e-\mu, e-3$ and $\mu-3$ final states, \\
& & \\
\hline Average & $(17.9 \pm 0.4) \%$ & assumes $e-\mu$ universality \\
\hline theoretical prediction & $(17.6 \pm 1.3) \%$ & standard model and $\tau_{\tau}$ \\
\hline
\end{tabular}

$$
\underline{r^{-}+\nu_{r} \mu^{-} L_{\mu s}}
$$

Taking into account the mass of the muion, the standard model predicts

$$
\frac{\Gamma\left(r^{-} \rightarrow \nu_{r} \mu^{-} \nu_{\mu}\right)}{\Gamma\left(r^{-}-\nu_{+} e^{-} \nu_{c}\right)}=1-8 y+8 y^{3}-y^{4}-12 y^{2} \ln y=0.972
$$

where $y=\left(\frac{m_{\mu}}{m_{t}}\right)^{2}$. Lsing the measured tau lifetime, the predicted branching fraction is $B\left(r^{-} \rightarrow \nu_{r} \mu^{-} \nu_{\mu}\right)=(17.0 \pm 1.3) \%$.

Published measurements of $B\left(r^{-} \rightarrow \nu_{\tau} \mu^{-} \nu_{\mu}\right)$ are listed in Table 1.5 along with the event topologies used in each measurement. Again the average measured value for $B\left(r^{-}-\nu_{\mathrm{r}} \mu^{-} L_{\mu}\right)$ is dominated by the recent precise measurements by MAC and Mark III, and agrees very weil with theory and the measured tau lifetime. 
Table 1.5. Published measurements of $P(\tau$

\begin{tabular}{|c|c|c|}
\hline Experiment & Branching Fraction & Method \\
\hline Mark II 24 & $(17.1 \pm 0.6 \pm 1.0) \%$ & $\begin{array}{c}\mu-e \text { final states, } \\
\text { assumes } e-\mu \text { universality }\end{array}$ \\
\hline PLUTO 8 & $(19.4 \pm \mathrm{I} .6 \pm 1.7) \%$ & $\mu-X$ final states, $X \neq \mu$ or $\pi$ \\
\hline TASSO $^{\circ}$ & $\left(12.9 \pm 1.7_{-0.5}^{+0.7}\right) \%$ & $\mu-X$ final states, $X \neq \mu$ \\
\hline CELLO 25 & $(17.6 \pm 2.6 \pm 2.1) \%$ & $\mu-X$ final states, $X \neq \mu$ \\
\hline Mark III 26 & $(18.0 \pm 1.0 \pm 0.6) \%$ & $\mu-e, \mu-\mu$ and $e-e$ final states \\
\hline $\mathrm{MAC}^{27}$ & $(17.3 \pm 0.5) \%$ & $\begin{array}{c}\mu-e, \mu-3 \text { and } e-3 \text { final states, } \\
\text { assumes } e-\mu \text { universality }\end{array}$ \\
\hline Average & $(17.2 \pm 0.4) \%$ & \\
\hline theoretical prediction & $(17.0 \pm 1.3) \%$ & standard model and $\mathrm{Tr}_{\mathrm{r}}$ \\
\hline
\end{tabular}

1.3.2 Semi-Leptonic Decay Modes

To determine the possible hadronic states to which the $W^{ \pm}$in tau decay can couple, it is necessary to know the quantum numbers carried hy the charged cursent. Since the $W^{-}$has one unit of electric charge it will couple to the quark-antiquark pair $\bar{u} d$ with one unit of charge and one unit of isospin $(I=1)$. Hence the $W^{ \pm}$carries one unit of isospin.

The weak charged current contains vector $\left(V_{\mu}\right)$ and axial-vector $\left(A_{\mu}\right)$ components. Sisce the vector current is conserved, its angular momentum can only be $J^{P}=1^{-}$and not $J^{P}=0^{+}$. Since the axial-vector current is noi sirictly conserved, it has angular momentum $J^{P}=0^{-}$or $1^{2}$.

The G-parity operator is defined to be $G=C e^{4 \pi / 2}$ where $C$ is the charge conjugation operator and $I_{2}$ is the second component of isospin. The vector component of the weak charged current has positive G-parity while the axial-vector component has negative G-parity. These quantem numbers are summarized in Table 1.6.

Table 1.6. Quantum numbers of the weak charged current.

\begin{tabular}{|c|c|c|}
\hline $\begin{array}{c}\text { Quantum } \\
\text { Number }\end{array}$ & $V_{\mu}$ & $\begin{array}{c}\text { Axial-Vector Component } \\
A_{\mu}\end{array}$ \\
\hline lsospin, & 1 & 1 \\
G-parity & + & - \\
$J^{P}$ & $1^{-}$ & $0^{-}, 1^{+}$ \\
\hline
\end{tabular}

Since the G-parity for a pion is negative, the G-parity for $n$ pions in $(-1)^{n}$. Thcrefore, the vector current couples to an even number of pions while the axialvector current couples to an odd number of pions.

In summ $r y$, the victor current couples to $n$ pions with $J^{G}=1^{+}, J^{P}=1^{-}$, where $n$ is even. The $n=2$ esonance corresponds to the $\rho$ meson. The $n=4$ resonance might be the $\rho(1600)$. The axial-vector current couples to $n$ pions with $I^{G}=1^{-}, J^{P}=0^{-}$or $1^{+}$, where $n$ is odd. The single pion $(n=1)$ has $J^{P}=0^{-}$. The $n=3$ resonance could correspond to the $A_{1}$ with $J^{P}=1^{+}$or the $\pi(1300)$ with $J^{P}=O^{-}$which is not a well-established resonance. Final states involving the $\eta$ $\left(m=549 \mathrm{MeV} / \mathrm{c}^{2}, I^{G}=0^{+}, J^{r}=0^{-}\right)$are also possible. For example, the vector current can couple to $\eta \pi \pi$ and the axial-vector current can couple to $\eta \pi \pi \pi$ or $\eta \eta \pi$. The final state $\pi \eta$ is forbidden since it has $I^{G}=1^{-}$and $J^{P}=0^{+}$or $1^{-}$. Each of 
these final states is discussed in the following sections.

$$
\underline{\tau^{-} \rightarrow \nu_{\tau} \pi^{-}}
$$

As shown in Figure 1.2, both $\tau^{-} \rightarrow \nu_{\tau} \pi^{-}$and $\pi^{-} \rightarrow \mu^{-} \nu_{\mu}$ involve the coupling of the weak axial-vector current to the pion. The decay widths for the two interactions are given by

$$
\Gamma\left(r^{-} \rightarrow \nu_{\tau} \pi^{-}\right)=\frac{G_{F}^{2}\left(\int_{\pi} \cos \theta_{c}\right)^{2} m_{r}^{3}}{16 \pi}\left(1-\frac{m_{-\pi}^{2}}{m_{r}^{2}}\right)^{2}
$$

and

$$
\Gamma\left(\pi^{-} \rightarrow \mu^{-} D_{\mu}\right)=\frac{G_{F}^{2}\left(f_{\pi} \cos \theta_{c}\right)^{2}}{8 \pi} m_{\pi} m_{\mu}^{2}\left(1-\frac{m_{\mu}^{2}}{m_{\pi}^{2}}\right)^{2}
$$

Therefore the coupling factor $f_{\pi} \cos \theta_{c}$ can be determined from the measured pion lifetime since the branching fraction for $\pi^{-} \rightarrow \mu^{-} E_{\mu}$ is $100 \%$. This results in

$$
\frac{\Gamma\left(r^{-} \rightarrow \nu_{+} \pi^{-}\right)}{\Gamma\left(\tau^{-} \rightarrow \nu_{\tau} e^{-} \nu_{e}\right)}=\frac{\left(\delta_{\pi} \cos \theta_{c}\right)^{2}}{m_{\tau}^{2}} 12 \pi^{2}\left(1-\frac{m_{\pi}^{2}}{m_{\tau}^{2}}\right)^{2}=0.607
$$

Using the measured tau lifetime, the predicted branching fraction is $B\left(\tau^{-} \rightarrow\right.$ $\left.\nu_{\tau} \pi^{-}\right)=(10.7 \pm 0.8) \%$.

Published measuiements of $B\left(\tau^{-} \rightarrow \nu, \pi^{-}\right)$are listed in Table i.7. Again the average agrees very well with the theoretical prediction and the measured taus lifetime.
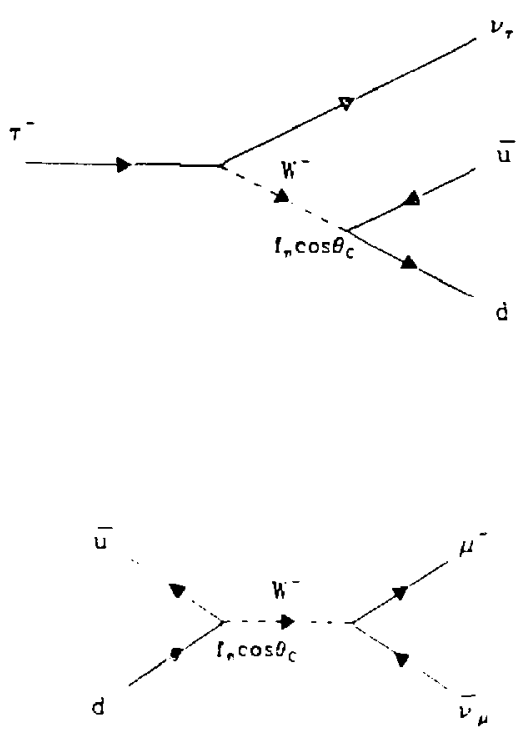
Table 1.7. Published measurements of $B\left(\tau^{-} \rightarrow \nu_{r} \pi^{-}\right)$.

\begin{tabular}{|c|c|c|}
\hline Experiment & Branching Fraction & Method \\
\hline Mark Il at SPEAR ${ }^{24}$ & $(11.7 \pm 0.4 \pm 1.8) \%$ & $\pi^{ \pm}-X^{\mp}$ \\
CELLO 25 & $(9.9 \pm 1.7 \pm 1.3) \%$ & $\pi-X$ \\
\hline Average & $(10.9 \pm 1.4) \%$ & \\
\hline theoretical prediction & $(10.7 \pm 0.8) \%$ & $\tau_{\pi}$ and $\tau_{r}$ \\
\hline
\end{tabular}

\section{$\underline{r}^{-} \rightarrow \underline{u_{t} \pi^{-} \pi^{0}}$}

All experimental evidence indicates that the $\pi^{-} \pi^{0}$ system in tau decay is dominated by the $\rho$ resonance. Since the $p$ has quantum numbers $J^{P}=1^{-}$, only the vector component of the weak current contributes to the decay $r^{-} \rightarrow \nu_{r} p^{-}$. Therefore, the conserved vector current (CVC) hypothesis can be used to relate the strength of the charged weak vector current coupling to $\pi \pi$ to that of the neutra electromagnetic vector current. The strength of the latter coupling is measured by the cross section $0\left(e^{+} e^{-} \rightarrow \gamma \rightarrow \pi^{+} \pi^{-}\right)$. The relationship between the two couplings is given by

$$
\frac{\Gamma\left(\tau^{-} \rightarrow \nu_{r} \pi^{-} \pi^{0}\right)}{\Gamma\left(\tau^{-} \rightarrow \nu_{\tau} e^{-} D_{e}\right)}=\frac{3}{2 \pi \alpha^{2} m_{\tau}^{8}} \int^{m_{r}^{2}} d Q^{2} Q^{2}\left(m_{\tau}^{2}-Q^{2}\right)^{2}\left(m_{\tau}^{2}+2 Q^{2}\right) \sigma_{e}+e^{-} \rightarrow \pi^{+} \pi^{-}\left(Q^{2}\right),
$$

where $Q$ is the center-of-mass energy of the $e^{+} e^{-}$system. Using the measured cross sections from $e^{+} e^{-}$storage rings, Gilman and Rhie calculate

$$
\frac{\Gamma\left(\tau^{-} \rightarrow \nu_{r} \pi^{-} \pi^{0}\right)}{\Gamma\left(\tau^{-} \rightarrow \nu_{T} e^{-} D_{e}\right)}=1.23
$$

Uaing the measured tau lifetime, the predicted branching fraction is $B\left(\tau^{-} \rightarrow\right.$ $\left.\nu_{\tau} \rho^{-}\right)=(21.6 \pm 1.5) \%$

Published values of $B\left(r^{-} \rightarrow \nu_{r} \rho^{-}\right)$are given in Table 1.8 and again there is good agreement between the experimental measurements and theory.

Table 1.8. Published measurements of $B\left(r^{-} \rightarrow \nu_{r} p^{-}\right)$.

\begin{tabular}{|c|c|c|}
\hline Experiment & Branching Fraction & Method \\
\hline Mark II at SPEAR ${ }^{28}$ & $(20.5 \pm 4.1) \%$ & $\rho-\epsilon$ and $\rho-\mu$ \\
CELLO $^{7}$ & $(22.1 \pm 1.9 \pm 1.6) \%$ & $\rho-X$ \\
Mark II & & $\rho-X$ \\
\hline Average & $(22.3 \pm 0.6 \pm 1.4) \%$ & \\
\hline theoretical prediction & $(22.1 \pm 1.2) \%$ & CVC and $\tau_{+}$ \\
\hline
\end{tabular}

\section{$\tau^{-} \rightarrow \nu_{\tau}(3 \pi)^{-}$}

The $(3 \pi)^{ \pm}$aystem is expected to be dominated by the $A,\left(J^{P}=1^{+}\right)$ and possibly the $\pi^{\prime}\left(J^{P}=0^{-}\right)$resonance through the axial vector coupling. Experimental evidence indicates that the $3 \pi^{ \pm}$final state corresponds to the decay products of a $J^{P}=1^{+}$state which decays to a $\rho \pi^{ \pm}$intermediate state in a relative j-wave. There are no experimental measurements which can be used to predict $\Gamma\left(r^{-} \rightarrow \nu_{r}(3 \pi)^{-}\right)$. However, Gilman and Rhie use isotopic spin considerations to make the following predictions about the relative branching fraction to one and th ree charged particles:

$$
\frac{1}{5} \leq \frac{\Gamma\left(r^{-} \rightarrow \nu_{r} \pi^{-} 2 \pi^{0}\right)}{\Gamma\left(\tau^{-} \rightarrow \nu_{r}(3 \pi)^{-}\right)} \leq \frac{1}{2}
$$


and

$$
\frac{1}{2} \leq \frac{\Gamma\left(\tau^{-} \rightarrow \nu_{r} \pi^{-} \pi^{+} \pi^{-}\right)}{\Gamma\left(\tau^{-} \rightarrow \nu_{r}(3 \pi)^{-}\right)} \leq \frac{1}{5}
$$

Therefore, the branching fraction to $\left(3 \pi^{ \pm}\right)$must be greater than or equal to the

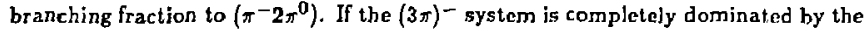
$A_{1}$, the branching fractions to one and three charged particles will be identical.

The published measurements of $B\left(+^{-} \rightarrow \nu_{r} \pi^{-} \pi^{+} \pi^{-}\right)$are listed in Table 1.9 . For the average, the contribution from the mode $\mathrm{T}^{-} \rightarrow \nu_{\pi} \mathrm{K}^{*-}$ is subtracted from the measurements for which this mode was not considered as a background.

Table 1.9. Published measurements of $B\left(+^{-} \rightarrow \nu_{+} \pi^{-} \pi^{+} \pi^{-}\right)$.

\begin{tabular}{|c|c|}
\hline Experiment & Branching Fraction \\
\hline CELLO $^{7}$ & $(9.7 \pm 2.0 \pm 1.3) \%$ \\
Mark II $^{30}$ & $(7.8 \pm 0.5 \pm 0.8) \%$ \\
MAC $^{11}$ & $(8.1 \pm 0.4 \pm 0.7) \%$ \\
DELCO $^{15}$ & $(5.0 \pm 1.0) \%$ \\
\hline Average & $(7.3 \pm 0.5) \%$ \\
\hline theoretical prediction & none \\
\hline
\end{tabular}

The single-charged-particle mode is more difficult to ineasure because of the large number of photons which must be detected. CELLO ${ }^{7}$ measuras $(6.0 \pm 3.0 \pm$ $1.8) \%$ for $B\left(r^{-}+\nu_{r} \pi^{-} 2 \pi^{0}\right)$ which is consistent with being equal to the branghing fraction for the three-charged-particle mode. $\underline{r^{-} \rightarrow \underline{\nu_{\tau}(4 \pi)^{-}}}$

The width for $\tau^{-} \rightarrow \nu_{r}(4 \pi)^{-}$can be related to the cross section for $e^{+} e^{-} \rightarrow 4 \pi$ assuming the decay proceeds via the vector current and assuming CVC. Gilman and Rhic have used recent data from $e^{+} e^{-}$storage sings to predict.

$$
\begin{gathered}
\frac{\Gamma\left(r^{-} \rightarrow \nu_{\tau} \pi^{-} 3 \pi^{0}\right)}{\Gamma\left(r^{-}+\nu_{\tau} e^{-} \nu_{e}\right)}=0.055, \\
\frac{\Gamma\left(r^{-} \rightarrow \nu_{\tau} \pi^{-} \pi^{+} \pi^{-} \pi^{0}\right)}{\Gamma\left(r^{-} \rightarrow \nu_{r} e^{-} \nu_{e}\right)}=0.275,
\end{gathered}
$$

and the sum

$$
\frac{\Gamma\left(r^{-} \rightarrow \nu_{\tau}(4 \pi)^{-}\right)}{\Gamma\left(r^{-} \rightarrow \nu_{\tau} e^{-} \nu_{e}\right)}=0.33
$$

Using the measured tau lifetime, the predicted branching fraction for the threecharged-particle mode is $B\left(\tau^{-} \rightarrow b_{\tau} \pi^{-} \pi^{+} \pi^{-} \pi^{0}\right)=(4.8 \pm 0.3) \%$. The published measurements for $B\left(\tau^{-} \rightarrow \nu_{r} \pi^{-} \pi^{+} \pi^{-} \pi^{0}\right)$ are listed in Table 1.10. Again, the singlecharged-particle mode is more difficult to measure because of the large number of photons which must be detected. CELLO $^{7}$ measures $(3.0 \pm 2.2 \pm 1.5) \%$ for $B\left(\tau^{-} \rightarrow \nu_{r} \pi^{-}-3 \pi^{0}\right)$. From the above relationships, $B\left(\tau^{-} \rightarrow \nu_{\tau} \pi^{-}-3 \pi^{0}\right)$ is predicted to be about $1 \%$ whirh is consistent with the measurement by CELLO.

$$
\underline{\tau^{-} \rightarrow v_{r}(5 \pi)^{-}}
$$

As with the decay $\tau^{-} \rightarrow \nu_{\tau}(3 \pi)^{-}$, there are no experimental measurenents which can be used to predict $\Gamma\left(r^{-} \rightarrow \nu_{r}(5 \pi)^{-}\right)$. Gilman and Rhie use isotopic spin considerations to predict the following relative branching fractions to one, three and 
Table 1.10. Published measurements of $B\left(\tau^{-} \rightarrow \nu_{r} \pi^{-} \pi^{+} \pi^{-} \pi^{0}\right)$.

\begin{tabular}{|c|c|}
\hline Experiment & Branching Fraction \\
\hline CELLO $^{7}$ & $(6.2 \pm 2.3 \pm 1.7) \%$ \\
Mark II $^{30}$ & $(4.7 \pm 0.5 \pm 0.8) \%$ \\
MAC $^{11}$ & $(5.2 \pm 0.4 \pm 0.7) \%$ \\
DELCO ${ }^{15}$ & $(6.0 \pm 1.2) \%$ \\
\hline Average & $(5.3 \pm 0.5) \%$ \\
\hline theoretical prediction & $(4,8 \pm 0.3) \%$, uses CVC and $\tau_{T}$ \\
\hline
\end{tabular}

five charged particles:

$$
\begin{aligned}
0 & \leq \frac{\Gamma\left(r^{-} \rightarrow \nu_{r} \pi^{-} 4 \pi^{0}\right)}{\Gamma\left(\tau^{-} \rightarrow \nu_{r}(5 \pi)^{-}\right)} \leq \frac{3}{10}, \\
\frac{8}{35} & \leq \frac{\Gamma\left(\tau^{-} \rightarrow \nu_{\tau} 3 \pi^{ \pm} \pm 2 \pi^{0}\right)}{\Gamma\left(r^{-} \rightarrow \nu_{\tau}(5 \pi)^{-}\right)} \leq 1, \\
0 & \leq \frac{\Gamma\left(\tau^{-} \rightarrow \nu_{\tau} 5 \pi^{ \pm}\right)}{\Gamma\left(\tau^{-} \rightarrow \nu_{r}(5 \pi)^{-}\right)} \leq \frac{24}{35} .
\end{aligned}
$$

This does not put any upper bound on the rate for $r^{-} \rightarrow \nu_{\tau} 3 \pi^{ \pm} 2 \pi^{0}$ in terms of the rate for $\tau^{-} \rightarrow \nu_{r}(5 \pi)^{-}$. All of this decay could go into the three-charged-particle mode.

The published measurements of the branching fraction to final states containing five charged particles and any number of neutrals, $B\left(\tau^{-} \rightarrow \nu, 5 \pi^{ \pm}\left(\pi^{0}\right)\right)$, are shown in Table 1.2. The average value is $(0.14 \pm 0.04) \%$. Gilman and Rhie use isospin conservation to show that

$$
\frac{\Gamma\left(r^{-} \rightarrow \nu_{+} \pi^{-} 4 \pi^{0}\right)}{\Gamma\left(\tau^{-} \rightarrow \nu_{r} 5 \pi^{ \pm}\right)} \leq \frac{3}{4}
$$

The average value for $B\left(\tau^{-} \rightarrow \nu_{r} 5 \pi^{ \pm}\left(\pi^{0}\right)\right)$ and the above relation result in the following limit on the one prong mode:

$$
B\left(\tau^{-} \rightarrow \nu_{r} \pi^{-} 4 \pi^{0}\right) \leq(0.11 \pm 0.03) \%
$$

$T^{-} \rightarrow \nu_{r}(6 \pi)^{-}$

Using isotopic spin conservation, Gilman and Rhie calculate the following relationships:

$$
\begin{aligned}
& 0 \leq \frac{\left(\Gamma\left(r^{-} \rightarrow \nu_{r} \pi^{ \pm} 5 \pi^{0}\right)\right.}{\Gamma\left(r^{-} \rightarrow \nu_{r}(6 \pi)^{-}\right)} \leq \frac{9}{35}, \\
& \frac{1}{5} \leq \frac{\Gamma\left(r^{-} \rightarrow \nu_{r} 3 \pi^{ \pm} 3 \pi^{0}\right)}{\Gamma\left(\tau^{-} \rightarrow \nu_{r}(6 \pi)^{-}\right)} \leq \frac{4}{5}, \\
& \frac{1}{5} \leq \frac{\Gamma\left(\tau^{-} \rightarrow \nu_{r} 5 \pi^{ \pm} \pi^{0}\right)}{\Gamma\left(\tau^{-} \rightarrow \nu_{r}(6 \pi)^{-}\right)} \leq \frac{4}{5} .
\end{aligned}
$$

This implies that

$$
\begin{aligned}
B\left(\tau^{-} \rightarrow \nu_{\tau}(6 \pi)^{-}\right) & \leq 5 B\left(\tau^{-} \rightarrow \nu_{\tau} 5 \pi^{ \pm} \pi^{0}\right) \\
& \leq 5 B\left(\tau^{-} \rightarrow \nu_{\tau} 5 \pi^{ \pm}\left(\pi^{0}\right)\right) .
\end{aligned}
$$

Thereforc, the experimental measurement of $B\left(r^{-} \rightarrow \nu_{r} 5 \pi^{ \pm}\left(\pi^{0}\right)\right)$ can be used to establish an upper bound of $(0.7 \pm 0.2) \%$ on the branching fraction for the complete mode $r^{-} \rightarrow \nu_{r}(6 \pi)^{-}$. Similarly,

$$
B\left(\tau^{-} \rightarrow \nu_{\tau} \pi^{ \pm} 5 \pi^{0}\right) \leq(9 / 7) B\left(\tau^{-} \rightarrow \nu_{\tau} 5 \pi^{ \pm} \pi^{0}\right)
$$

$$
\leq(9 / 7) B\left(\tau^{-} \rightarrow \nu_{r} 5 \pi^{ \pm}\left(\pi^{0}\right)\right)
$$


resulting in an upper bound of $(0.18 \pm 0.05) \%$ on the branching fraction for the one-charged-particle mode $\tau^{-} \rightarrow \nu_{\tau} \pi^{ \pm} \pi^{0}$. Also,

$$
B\left(\tau^{-} \rightarrow \nu_{\tau} 3 \pi^{ \pm} 3 \pi^{0}\right) \leq 4 B\left(\tau^{-} \rightarrow \nu_{T} 5 \pi^{ \pm}\left(\pi^{0}\right)\right)
$$

resulting in an upper bound of $(0.56 \pm 0.16) \%$ on the branching fraction for the three-charged-particle mode $\tau^{-} \rightarrow \nu_{r} 3 \pi^{ \pm} 3 \pi^{0}$.

$$
I^{-} \rightarrow \nu_{r} K^{-}
$$

The decay width for $\tau^{-} \rightarrow \nu_{r} K^{-}$is given by

$$
\frac{\Gamma\left(\tau^{-} \rightarrow \nu_{r} K^{-}\right)}{\Gamma\left(\tau^{-} \rightarrow \nu_{\tau} e^{-} \nu_{e}\right)}=\frac{\left(f_{K} \sin \theta_{c}\right)^{2}}{m_{\tau}^{2}} 12 \pi^{2}\left(1-\frac{m_{K}^{2}}{m_{\tau}^{2}}\right)^{2}
$$

As shown in Figure 1.3, both $\tau^{-} \rightarrow \nu_{r} K^{-}$and $K^{-} \rightarrow \mu^{-} \nu_{\mu}$ involve the coupling of the axial-vector current to the kaon. Therefore the factor $f_{K} \sin \theta_{c}$ can be determined from the dominant decay of the charged kaon. This results in

$$
\frac{\Gamma\left(\tau^{-} \rightarrow \nu_{r} K^{-}\right)}{\Gamma\left(\tau^{-} \rightarrow \nu_{r} e^{-} \nu_{e}\right)}=0.0395
$$

Using the measured tau ljfetime, the predicted branching fraction is $(0.69 \pm 0.05) \%$. Published measurements of $B\left(\tau^{-} \rightarrow \nu_{\tau} K^{-}\right)$are shown in Table 1.11. In the DELCO experiment, information from Cerenkov and time-of-flight counters is used to identify the charged kaons. In the Mark II experiment at SPEAR, only time-offlight information is used to idestify the kaons.
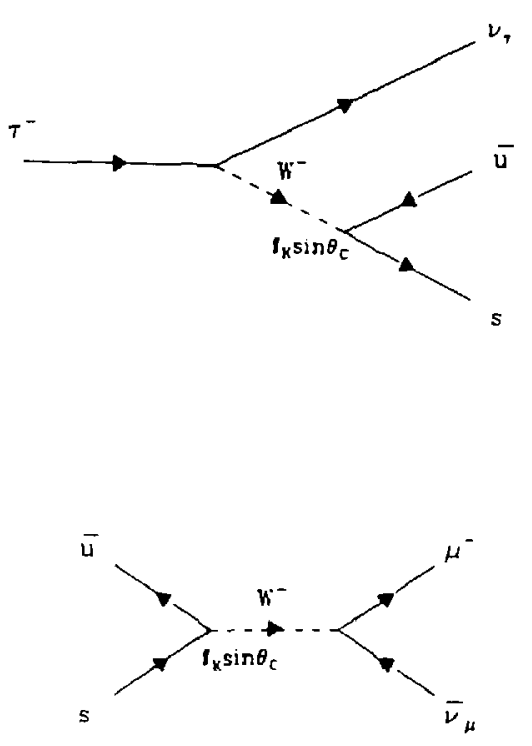
Table 1.11. Published measurements of $B\left(\tau^{-} \rightarrow \nu_{\uparrow} K^{-}\right)$.

\begin{tabular}{|c|c|}
\hline Experiment & Branching Fraction \\
\hline Mark II at SPEAR $^{31}$ & $(1.3 \pm 0.5) \%$ \\
DELCO $^{32}$ & $(0.59 \pm 0.18) \%$ \\
\hline Average & $(0.67 \pm 0.17) \%$ \\
\hline theoretical prediction & $(0.69 \pm 0.05) \%$ \\
\hline
\end{tabular}

\section{$\underline{\tau^{-} \rightarrow \nu_{\tau} K^{\star-}}$}

The decay rate for $\tau^{-} \rightarrow \nu_{T} K^{*-}$ can be determined from that for $\tau^{-} \rightarrow \nu_{\tau} \rho^{-\infty}$ by multiplying by $\tan ^{2} \theta_{c}$ due to the strangeness-changing current and by a phase is space factor. The result is

$$
\frac{\Gamma\left(r^{-} \rightarrow \nu_{r} K^{*-}\right)}{\Gamma\left(\tau^{-} \rightarrow \nu_{r} e^{-} \nu_{e}\right)}=0.079
$$

Using the measured tau lifetime, the predicted branching fraction is $(1.39 \pm 0.10) \%$. Published measurements of $B\left(\tau^{-} \rightarrow \nu_{\mathrm{r}} \mathrm{K}^{\bullet-}\right)$ are shown in Table 1.12. Both of these meaurements use the decay $K^{0-} \rightarrow \pi^{-} K_{S}^{0}$ as a signature, identifying $\pi^{+} \pi^{-}$ pairs from the decay of the $K_{S}^{D}$.

Table 1.12. Published measurements of $B\left(r^{-} \rightarrow \nu_{\tau} K^{\bullet-}\right)$.

\begin{tabular}{|c|c|}
\hline Experiment & Branching Fraction \\
\hline Mark II at SPEAR ${ }^{34}$ & $(1.7 \pm 0.7) \%$ \\
Mark Il at PEP ${ }^{29}$ & $(1.3 \pm 0.3 \pm 0.3) \%$ \\
\hline Average & $(1.4 \pm 0.3) \%$ \\
\hline theoretical prediction & $(1.39 \pm 0.10) \%$ \\
\hline
\end{tabular}

1.3.3 Modies wilh an $\eta$ in the Final State

The possible decay modes with an $\eta$ in the final state are summarized in Table 1.13 along with the quant um numbers and possible identity of the rescnance. Since the $\eta$ decays to two charged particles approximately $30 \%$ of the time, ${ }^{35}$ the decay $\tau^{-} \rightarrow \nu_{\mathrm{r}} \pi^{-} \eta \pi^{+} \pi^{-}$results in five charged particles in the final state $30 \%$ of the time and the decay $\tau^{-} \rightarrow \nu_{\tau} \pi^{-} \eta \eta$ results in five charged particles $9 \%$ of the time. Assuming the efficiency for detecting these final states is the same as the efficiency used in the measurements of the branching fraction for $r^{-} \rightarrow \nu_{r} 5 \pi^{ \pm}\left(\pi^{0}\right), *$ the following limits can be set:

$$
\begin{gathered}
B\left(\tau^{-} \rightarrow \nu_{\tau} \pi^{-} \eta \pi^{+} \pi^{-}\right) \leq B\left(\tau^{-} \rightarrow \nu_{r} 5 \pi^{ \pm}\left(\pi^{0}\right)\right) / 0.3 \approx 0.5 \%, \\
B\left(\tau^{-} \rightarrow \nu_{r} \pi^{-} \eta \eta\right) \leq B\left(\tau^{-} \rightarrow \nu_{r} 5 \pi^{ \pm}\left(\pi^{0}\right)\right) / 0.09 \approx 1.5 \% .
\end{gathered}
$$

Isospin conservation predicts $B\left(\tau^{-} \rightarrow \nu_{\mathrm{r}} \pi^{-} \eta \pi^{0} \pi^{0}\right) \leq B\left(\tau^{-} \rightarrow \nu_{\mathrm{r}} \pi^{-} \eta \pi^{+} \pi^{-}\right)$.

Finally, a limit can be set on the branching fraction for the mode ${ }^{-} \rightarrow$ $\nu_{r} \pi^{-} \eta \pi^{0}$ assuming it occurs via the $\rho(1600)$ resonance. The branching fraction

* The efficiency might be lower for the modes involving $\eta$ 's since the dominant decay of the $\eta$ to two charged parteles is $\eta \rightarrow \pi^{+} \pi^{-} \pi^{0}$ in which the mass of the final state particlos is very close to the mass of the $\eta$ resulting in a small opening angle between the $\pi^{+}$and the $\pi^{-}$in the laboratory frame. Small opening angles between the charged tracks contributed strongly to tracking inefficiency in the Mark II analysis of $r^{-} \rightarrow \nu, 5 \pi^{t}\left(\pi^{0}\right)$ but not as strongly in the HRS analysis because of the higher magnetic field in the HRS detector. 
Table 1.13. Possible decay modes with an $\eta$ in the final state.

\begin{tabular}{|c|c|c|c|}
\hline$\tau^{-} \rightarrow \nu_{r}$ & $I^{G}$ & $J^{P}$ & Possible Rebonance \\
\hline$\pi^{-} \eta \pi^{0}$ & $1^{+}$ & $1^{-}$ & $\rho(1600)$ \\
\hline$\pi^{-} \eta \pi^{+} \pi^{-}$ & $1^{-}$ & $1^{+}$ & $A_{1}$ \\
$\pi^{-} \eta \pi^{0} \pi^{0}$ & & & \\
\hline$\pi^{-} \eta \eta$ & $1^{-}$ & $0^{-}$ & $\pi(1300)$ \\
& & $1^{+}$ & $A_{1}$ \\
\hline
\end{tabular}

of the $\rho(1600)$ to $\pi^{-} \eta \pi^{0}$ is approximately $7 \%$ and to $4 \pi$ is approximately $60 \% .^{35}$ Assuming a total branching fraction of the $\tau$ to $4 \pi$ of $6 \%$, as predicted by Gilman and Rhie, the branching fraction of the $\tau$ to $\pi^{-} \eta \pi^{0}$ is approximately $\frac{0.07}{0.60} \times 6 \%$ which is equal to $0.7 \%$. Therefore, the modes involving $\eta$ 's are expected to contribute a few percent, at the most, to the total decay rate.

\subsubsection{Other Decay Modes}

The DELCO experiment has meausured decay rates for final states containing one charged kaon and any number of neutral pions. ${ }^{32}$ The result is

$$
B\left(\tau^{-} \rightarrow \nu_{\uparrow} K^{-}+n \pi^{0}, n \geq 0\right)=(1.71 \pm 0.29) \%
$$

This includes the decay $\tau^{-} \rightarrow \nu_{r} K^{-}$and some of the final states for the decay $\tau^{-} \rightarrow \nu_{r} K^{\bullet-}$

The DELCO experiment has also measured decay rates for final states containing three charged particles in which one or more of the charged particles is identified as a kaon. ${ }^{36}$ The results are

$$
B\left(r^{-} \rightarrow \nu_{\tau} K^{-} K^{+} \pi^{-}\right)=\left(0.22_{-0.11}^{+0.17}\right) \%
$$

and

$$
B\left(\tau^{-} \rightarrow \nu_{\tau} K^{-} \pi^{+} \pi^{-}\left(\pi^{0}\right)\right)=(0.22+0.16) \%
$$

The first mode is expected to proceed via the $\rho(1600)$ resonance. The theoretical prediction for this decay rate, assuming CVC and $B\left(\rho(1600) \rightarrow K^{*} K\right)=(9 \pm 2) \%$, is $0.24 \%$. The second mode is expected to proceed via ine $Q$ resonances. The theoretical prediction for this decay rate is $0.11 \%$. The TPC ${ }^{33}$ experiment publisterd an upper limit of $0.6 \%$ at the $90 \%$ confidence level on the branching fraction for the decay $\tau^{-} \rightarrow\left(K^{-}+2\right.$ charged particles + any number of neutrals $)$.

The Mark il experiment at SPEAR set an -pper limit of $0.9 \%$ at the $95 \%$ confidence level for the decay $\tau^{-} \rightarrow \nu_{r} K^{*-}(1430) .^{34}$

In addition, the Mark II experinent at SPEAR conducted a search for twelve neutrinoless decay modes of the tau which violate lepton-number conservation. ${ }^{37}$ These modes include the radiative decays $\tau \rightarrow$ er and $r \rightarrow \mu \gamma ;$ the charged lepton decays $\tau \rightarrow e e c, \tau \rightarrow e e \mu, \tau \rightarrow \mu \mu e$ and $r \rightarrow \mu \mu \mu_{i}$ and the charged lepton plus neutral hadron decays $\tau \rightarrow e \rho, \tau \rightarrow \mu \rho, \tau \rightarrow e K^{0}, \tau \rightarrow{ }_{i} t K^{0}, \tau \rightarrow e \pi^{0}$ and $\tau \rightarrow \mu \pi^{0}$. No evidence for lepion-number violation was observed. For each of the first eight decay modes, an upper limit of less than $0.1 \%$ at the $90 \%$ confidence level was established. For the last four decay modes, upper limits of $0.2 \%$ or less were established. 
1.4 THE DISCREPANCY

The measured branching fractions for all of the above decay modes are listed in Table 1.14 according to the number of charged particles in the final state. The sum of the branching fractions for modes resulting in three charged particles is $(13.3 \pm 0.8) \%$. This is in such good agreement with the inclusive branching fraction to three charged particles since the measurement of $B\left(\tau^{-} \rightarrow \nu_{\mathrm{r}} \pi^{-} \pi^{+} \pi^{-} \pi^{0}\right)$ and $B\left(\tau^{-} \rightarrow v_{T} \pi^{-} \pi^{+} \pi^{-}\right\}$is usually performed by dividing all the three charged prong decays into those with and without neutrals.

The sum of the branching fractions for the exclusive decay modes which result in one charged particle is $(78.9 \pm 4.8) \%$. Recall that the inclusive brai-hing fraction to one charged particle, $B 1$, is $(86.8 \pm 0.3) \%$. The difference is $(7.9 \pm 4.8) \%$. Most of the error in the sum of the exclugive branching fractions is due to the measurements for $B\left(\tau^{-} \rightarrow \nu_{r} \pi^{-} 2 \pi^{0}\right)$ and $B\left(\tau^{-} \rightarrow \nu_{\tau} \pi^{-} 3 \pi^{0}\right)$. If we assume that $B\left(\tau^{-} \rightarrow\right.$ $\left.\nu_{\mathrm{r}} \pi^{-} 2 \pi^{0}\right)$ is equal to $B\left(r^{-} \rightarrow \nu_{\mathrm{r}} \pi^{-} \pi^{+} \pi^{-}\right)$, which is true according to isospin rules if the $3 \pi$ state is purely the $A_{1}$ resonance, and that $B\left(r^{-} \rightarrow \nu_{\tau} \pi^{-} 3 \pi^{0}\right)=1.0 \%$, which is the result of using CVC and $e^{t} e^{-}$cross section measurements to $4 \pi$ final states, then the sum of the exclusive one prong branching fractions is $(79.0 \pm 2.1) \%$ and the difference between the inclusive and the sum of the exclusive branching fractions to one charged particle is $(7.8 \pm 2.1) \%$, a 3.5 sigma effect.

One explanation for this discrepancy is that there exists an 'invisible' decay mode; i.e., a decay mode which is undetectable in existing detectors. One type of decay mode which can escape detection, for example, is the decay of the tau to
Table 1.14. Branching fractions for all decay modes (in per cent) according to charged particle multiplicity. A dash indicates that the decay mode is not expected to result in that charged multiplicity. The theoretical predictions assume a tau lifetime of $(2.8 \pm 0.2) \times 10^{-13} \mathrm{~s}$ and the errors on the predictions are those due to the error on the lifetime measurement only.

\begin{tabular}{|c|c|c|c|c|}
\hline Decay Mode & Experime & A. zsurements & Theorelica & redictions \\
\hline$r^{-} \rightarrow$ & 2 prong & 3 prong & 1 prong & 3 prong \\
\hline$e^{-} \tau_{e} \nu_{\tau}$ & $17.9 \pm 0.4$ & - & $17.6 \pm 1.3$ & - \\
\hline$\mu \cdot \nu_{\mu} \nu_{\tau}$ & $17.2 \pm 0.4$ & - & $17.0 \pm 1.3$ & - \\
\hline$\pi^{-} \nu_{\tau}$ & $10.9 \pm 1.4$ & - & $10.7 \pm 0.8$ & - \\
\hline$\pi^{-} \pi^{0} \nu_{r}$ & $22.1 \pm 1.2$ & - & $21.6 \pm: .5$ & - \\
\hline$(3 \pi)^{-} \nu_{\tau}$ & $6.0 \pm 3.5$ & $7.3 \pm 0.5$ & $\leq x$ & $x$ \\
\hline$(4 \pi)^{-} \nu_{r}$ & $3.0 \pm 2.6$ & $5.3 \pm 0.5$ & $1.0 \pm 0.1$ & $4.8 \pm 0.3$ \\
\hline$(5 \pi)^{-} v_{t}$ & & & $<0.11$ & $z$ \\
\hline$(6 \pi)-\nu_{r}$ & & & $<0.1 k$ & $<0.56$ \\
\hline$K^{-} \nu_{\mathrm{T}}$ & $0.7 \pm 0.2$ & - & $0.69 \pm 0,05$ & - \\
\hline$(K \pi)^{-} \nu_{r}$ & $1.1 \pm 0.3$ & $0.3 \pm 0.1$ & $1.08 \pm 0.08$ & $0.31 \pm 0.02$ \\
\hline$(K \bar{K})^{-} \nu_{\tau}$ & & & $<1.2$ & $<0.6$ \\
\hline$(K \bar{K} \pi)^{-} u_{r}$ & & $0.22_{-0.11}^{+0.17}$ & $<1.29 y$ & $y$ \\
\hline$(K \pi \pi)^{-}\left(\pi^{0}\right) \nu_{r}$ & & $0.22_{-0.13}^{+0.16}$ & $<0.810$ & $w$ \\
\hline TOTAL & $78.9 \pm 4.8$ & $13.3 \pm 0.8$ & & \\
\hline
\end{tabular}

a heavy neutral which does not leave a signal in the detector and a light charged particle. If the neutral is heavy enough, the momentum of the charged particle is very small, even in the laboratory frame. If the momentum of the charged particle is too small, the particle will curl up in the magnetic field without meeting the trigges requirements. However, this surt of explanation has been ruled out experimentally 
by the measurements of the total cross eection for $e^{+} e^{-} \rightarrow{ }^{+} \tau^{-}(\gamma)$. Published measurements of the ratio of the measured to the expected $Q E D$ cross section are listed in Table 1.15. The measured cross section is certainiy consistent with the QED cross section which is used for most branching fraction measurements. These cross section measurements are typically based on a sample of events with one charged particle in one hemisphere of the event and one or more charged particles in the opposite hemisphere. Therefore, if there is an invisible decay mode of the tau, events containing this decay mode will not satisfy the criteria for the cross section measurement sample and hence the measured cross section will be low. Also, events in which one tau decays into a detectable final suate, while the other tau decays into an undetectable final state, would have been fourd in recent searches for monojets and supersymmetric particles at PEP and PETRA

Table 1.15. Published measurements of the ratio of the measured to the expected QED cross section for $e^{+} e^{-} \rightarrow \tau^{+} \tau^{-}(\tau)$.

\begin{tabular}{|c|c|}
\hline Experiment & $\sigma_{\text {mecsured }} / \sigma_{\text {QED }}$ \\
\hline Mark II $^{38}$ & $0.996 \pm 0.016 \pm 0.028$ \\
HRS $^{39}$ & $1.10 \pm 0.03 \pm 0.04$ \\
PLUTO $^{8}$ & $0.89 \pm 0.05 \pm 0.08$ \\
TASSO $^{9}$ & $1.03 \pm 0.05_{-0.11}^{+0.06}$ \\
JADE $^{14}$ & $0.553 \pm 0.017 \pm 0.035$ \\
\hline Average & $1.02 \pm 0.03$ \\
\hline
\end{tabular}

1.5 GOAL OF THIS A NALYSIS

The goal of this analysis is to measure the branching fractions for all decay modes of the tau simultaneously by performing a maximum likelihood fit to an unbiased sample of tau decays. With this method, the sum of the measured branching fractions must be unity.

Most of the measurements listed in the previous section * were made using the following formula,

$$
B_{i}=\frac{n_{i} / c}{2 \times \sigma\left(e^{+} e^{-} \rightarrow \tau^{+} \tau^{-}\right) \int f d t},
$$

where $n_{i}$ is the number of events observed, $c$ is the efficiency or acceptance, $\sigma\left(e^{-1} e^{-} \rightarrow T^{+} T^{-}\right)$is the QED cross section for tau-pair production, and $\int \mathcal{L} d t$ is the integrated luminosity. The efficiency, $\epsilon$, is usually determined from Monte Carlo simulations and involyes two factors: the efficiency for extracting tau-pair events from other types of events in the data, and the efficiency for discriminating a particular decay mode from all the others.

A simplified version of the formula used to determine the branching fraction for decay mode $i$ in the method used in this analysis is

$$
B_{1}=\frac{n_{i} / \epsilon}{n_{\ell o l}}
$$

where $n_{i}$ is the number of events observed for decay mode $i_{i} \in$ is the particle identification efficiency for decay mode $i$, and $n_{\ell a t}$ is the total number of tau decays in the sample. It does not involve the efficiency for extracting tau decays from the

- Exceptions are the measurement of the leptonic branching fractions by $\mathrm{MAC}_{1}{ }^{27}$ and the branching fractions by PLUTO. ${ }^{8}$ 
data. Also, it does not involve the eross section for tau-pair production or the total integrated luminosity.

\section{The Mark II Detector}

2.1 OVERVIEW

The Mark II detector was designed and built by a collaboration of physicists, engineers and technicians from the Stanford Linear Accelerator Center (SLAC) and the Lawrence Berkeley Laboratory (LBL). It was installed at the small storage ring SPEAR at SLAC in 1977 where it was used to accumulate data from $\mathrm{e}^{+} \mathrm{e}^{-}$ annihilations at center of mass energies between 3.0 and $7.4 \mathrm{GeV}$. In the summer of 1979 the detector was : :nved to the larger storage ring PEP which was just being completed. A pproximately $200 \mathrm{pb}^{-1}$ of data were accumulated at PEP at a centerof-mass energy of $29 \mathrm{GeV}$. The detector was moved out of the PEP beamline in the spring of 1984 to be upgraded for $Z^{0}$ physics at the SLAC Linear Collider (SLC). The data accumulated at PEP are used in the analysis presented in this thesis.

As shown in Figure 2.1, the major components of the Mark II detector are a drift chamber surrounded by a solenoidal magnet to provide charged par ticle tracking and momentum measurement, a lead - liquid argon calorimeter to provide electron and photon jdentification, and layers of iron hadron absorber and proportional tubes to provide muon identification. Scintillators outside the drift chamber provide limited particle identification through time-of-flight measurements. In addition, a small high resolution drift chamber inside the main drift chamber is used to increase momentum resolution and measure particle lifetimes. Endcap calorimetcrs and a small angle tagging systern cover Bome of the areas not covered by the $\mathrm{iA}$ system.

In the following sections, each component of the detector will be discribed in 
detail. The role of each component in the particle identification algorithms will be described in the next chapter.

\subsection{VERTEX ChAMBER}

A high precision drift chamber, usually referred to as the vertex chamber (VC),

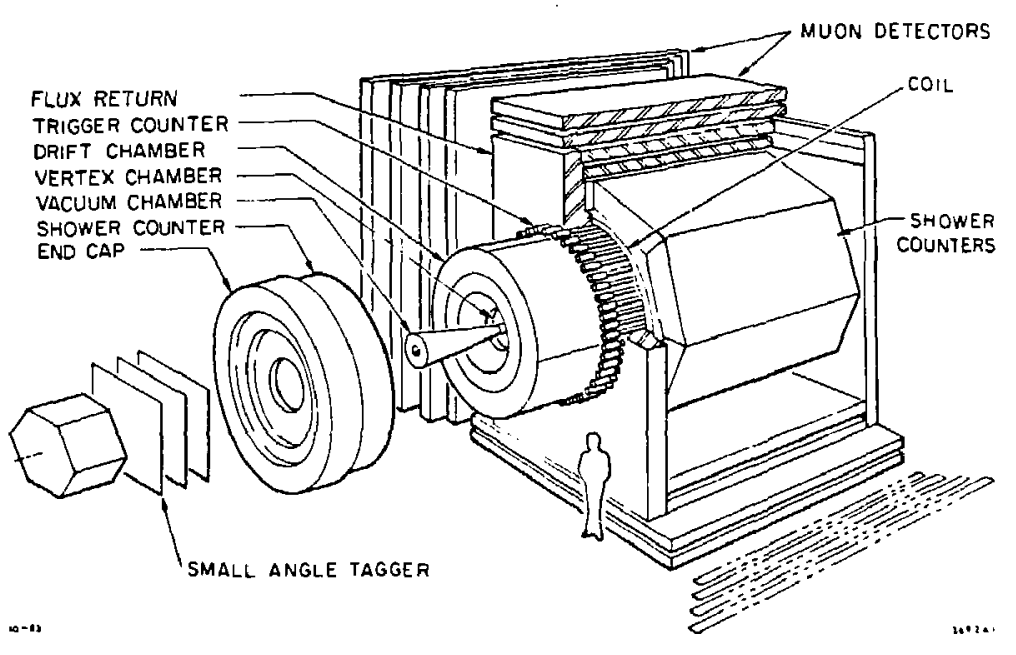
was installed inside the large central drift chamber in the slimmer of 1981. All data used in this analysis were accumulated with the vertex chamber installed. The VC was designed to measure lifetimes of weakly decaying farticles by reconstructing secondary vertices, an objective which has been successfully realized. It was also used in the primary and secondary triggers. The main influence of the VC in this analysis is the improved momentum resolution for charged tracks.

A side view of the $V C$ is shown in Figure 2.2. The chamber is $1.2 \mathrm{~m}$ long. To reduce the amount of multiple Coulomb scattering due to charged tracks passing through material, the inner cylindrical wall is also the vacuum beam-pipe wall. The pipe is made of beryllium to reduce the number of radiation lengths through which a particle passes. The pipe wall is $1.42 \mathrm{~mm}$ thick, the thickness required to hold off the pressure differential between the chamber and the vacuum beam pipe. The radius of the bearn pipe is $7.8 \mathrm{~cm}$, closen to be outside the envelope of synchrotron radiation produced by the final focusing quadropoles.

The sense wires are arranged in seven layers, four close to the inner cylinder to minimize the extrapolation error, and three near the outer cylinder to maximize the accuracy of the track angle measurement. The radii of the seven sense layers Figure 2.l. The Mark II detector at PEP. and the number of sense wires in each layer are listed in Table 2.1. The wire array 
is illustrated in Figure 2.j. Field layers containing only cathode wires at a negative high voltage alternate with sense layers containing cathode wires and sense wires at ground. An extra field layer inside and outside the array balances the gain on the edge sense wires. The distance between layers is $4.22 \mathrm{~mm}$ and the arc length between adjarent wires in the same layer is $5.30 \mathrm{~mm}$. This array produces a closed cell geometry which results in a simple relationship between drift time and distance of closest approach to the wire.

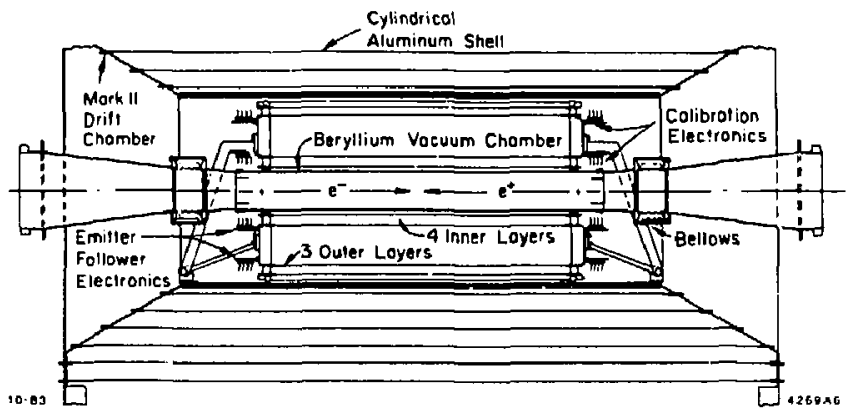

Table 2.1. Radii of sense wire layers in the vertex chamber and number of sense wires in each layer.

\begin{tabular}{|c|c|c|}
\hline Layer & $\begin{array}{c}\text { Radius } \\
(\mathrm{cm})\end{array}$ & $\begin{array}{c}\text { Number of } \\
\text { Sense Wires }\end{array}$ \\
\hline 1 & 10.1223 & 60 \\
2 & 10.9658 & 65 \\
3 & 11.8093 & 70 \\
4 & 12.6528 & 75 \\
\hline 5 & 30.3668 & 180 \\
6 & 31.2105 & 185 \\
7 & 32.0538 & 190 \\
\hline
\end{tabular}

The resulting spatial resclution is approximately $110 \mu \mathrm{m}$ per sense wire. The accuracy with which a single track can be extrapolated to the interaction point in the plane perpendicular to the beam is about $\sigma^{2}=(95 \mu \mathrm{m})^{2}+(95 \mu \mathrm{m} \mathrm{GeV} / p)^{2}$ where the first term is due to the limited resolution of individual drift-time measurements and the second term is due to multiple scattering in the beam pipe.

Figure 2.2. The Mark II vertex drift chamber. 


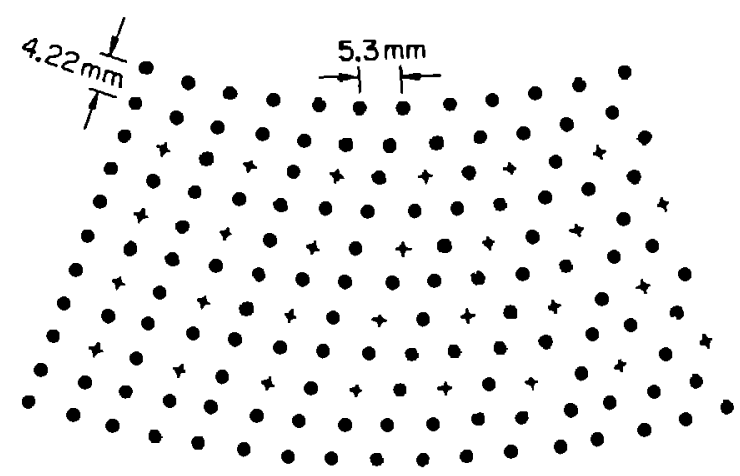

$\mathbf{3}-82$

- FIELD Wire + Sense Wire

4258A11

\subsection{MAIN DRIFT ChAMBER}

A cylindrical drift chamber with 16 layers of sense wires at radii between $0.41 \mathrm{~m}$ and $1.45 \mathrm{~m}$ provides charged particle tracking over $85 \%$ of the solid angle. The active length of the outer layer is about $2.64 \mathrm{~m}$. The wires in 6 of the layers are paraliel to the beam axis, while those in the remaining 10 layers are skewed at $\pm 3^{\circ}$ to the beam axis to provide spatial information in the direction of the axis. The wire array is illustrated in Figure 2.4.

The spatial resolution per wire is $\approx 200 \mu m$ resulting in a momentum resolution in the plane perpendicular to the beam axis of $(\delta p / p)^{2} \approx(0.02)^{2}+(0.01 p)^{2}$ where $p$ is in units of $G e V / c$, assuming a magnetic field of $2.32 \mathrm{kG}$.

\subsection{TIME-OF-FLIGHT SYSTEM}

The time-of-fight (TOF) system is located outside the drift chamber and inside the magnet coil at a radius of $1.5 \mathrm{~m}$. Forty-eight 1 inch thick Pilot $F$ scintillators parallel to the beam axis measure the time between the beam crossing and particle traversal to provide information for the primary and secondary triggers, cosmic ray rejection, and limited particle identification for low momentum tracks when combined with the momentum information from the drift chamber. The timing resolution of the TOF system when only ou.e particle traverses the scintillator is approximately $350 \mathrm{ps}$.

\subsection{SOLENOIDAL MIAGNET}

The conventional solenoidal magnet consists of two layers of water cooled 


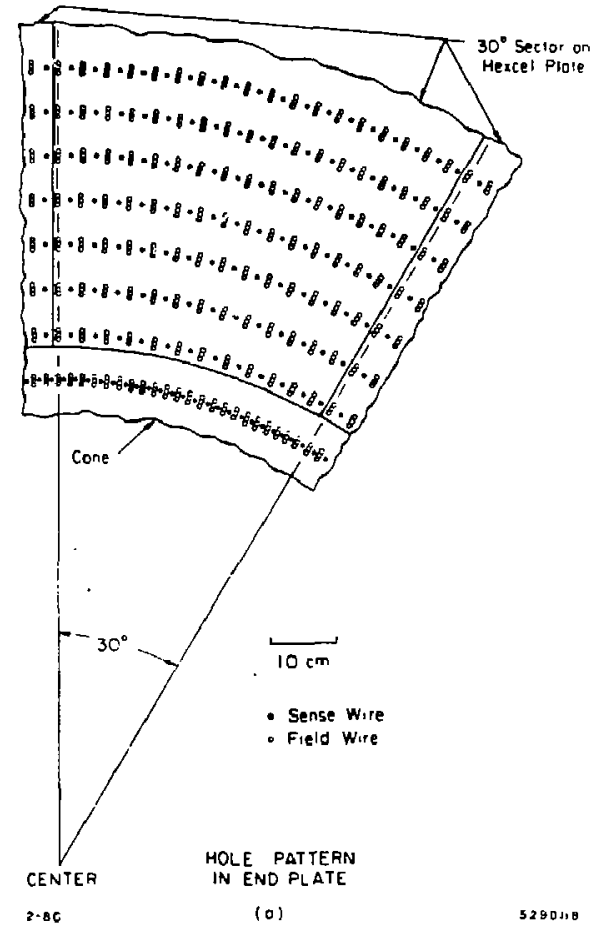

Figure 2.4. Wire array for main drift chamb. $\therefore$
During the winter data run of 198I-82, an electrical short developed between the two layers of conductors in the magnet. The magnet could then only $L$ a operated with the outer winding powered and the inner one floating. This results in a field of $2.32 \mathrm{kG}$. Almost all of the data analysed in this thesis was accumulated with the magnet operating in the half-field configuration.

\subsection{LEAD - Liquid ARGON Electromagnetic Calorimeter}

Surrounding the magnet are eight electromagnetic calorimeter modules $[3.8 \mathrm{~m}$ long $\times 1.8 \mathrm{~m}$ wirle $; 0.3 \mathrm{~m}$ deep) arranged in an octagonal array covering $\approx 65 \%$ of the solid angie. The modules consist of 37 layers of $2 \mathrm{~mm}$ thick antimony strengthened lead planes separated by $3 \mathrm{~mm}$ liquid argon gaps. Alternating lead plancs are at ground or segmented into readout st ${ }_{4}$ ips at a positive high voltage of $3.5 \mathrm{kV}$, resulting in 18 readout layers in depth. About $20 \%$ of the energy is deposited in the liquid argon. The total thickness of the calorimeter is about 14.5 radiation lengths.

The 18 readout planes are divided into strips as follows.

- F planes: 9 of the 18 planes are divided in to 40 strips of $3.49 \mathrm{~cm}$ width running parallel to the bean axis and thus providing spatial information for the azimuthal angle $\phi$.

- T planes: 6 of the 18 planes are divided into 100 strips of $3.49 \mathrm{~cm}$ width running perpendicular to the $\boldsymbol{F}$ strips and thus providing spatial information fo: the polar angle $\theta$.

- $U$ planes: 3 of the 18 planes are divided into 66 strips of $4.94 \mathrm{~cm}$ width 
runnir.g at $45^{\circ}$ to the $F$ and $T$ strips and thus providing multi-hit ambiguity

resolution.

Each strip is separated from its horizontal neighbors by $3 \mathrm{~mm}$ gaps.

Some of the strips in the same position in different layers with the same orientation are ganged together to reduce the number of readout layers in depth from 18 to 6 . The resulting layers are referred to as $F 1, F 2, F 3, T 1, T 2$, and $U$. This ganging scheme is shown in Figure 2.5.

In the T2 layer, adjacent sets of strips which are ganged in depth are also ganged horizontally in pairs since spatial resolution is not important for this coordinate so deep in the shower. In the U layer, the last ten strips at each end are also ganged horizontally in pairs.

At the front of each module there are three aluminum planes separated by E $\mathrm{mm}$ liquid argon gaps. The central plane is divided into $3.8 \mathrm{um}$ wjde readout strips running parallel to the beam axis. This gap, known as the trigger gap, samples showers which begin in the 1.25 radiation lergth magnet coil.

The overall energy resolution of the system is $\sigma_{E} / E=0.14 E^{-1 / 2}$ where $E$ is the energy in GeV.

\subsection{MUON IDENTIFICATION SYSTEM}

The muon identification system consists of four walls of alternating layers of iron hadron absorber and proportional tubes. The walls are located at. , s, below and on either side of the cetıtral detector. Each wall consists of four layers of proportional tubes. The outer layer of proportional tubes covers $45 \%$ of the solid

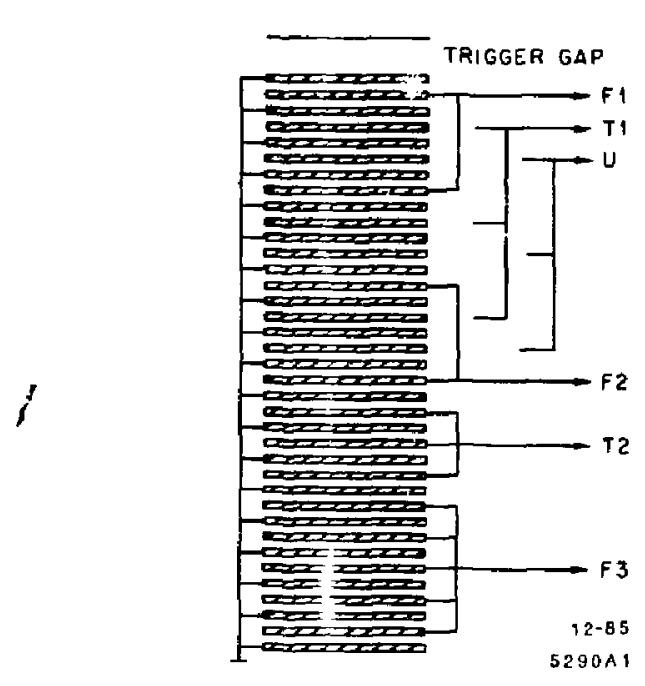

Figure 2.5. Ganging scheme in the $\mathrm{LA}$ calorimetes modules. Incident particles enter from the top. 
angle. The absorber thickness preceding each layer of tubes is shown in Table 2.2. The minimum momentum required for a muon traversing the absorber planes at normal incidence to penetrate all four levels is $\approx 1.8 \mathrm{GeV} / \mathrm{c}$. The proportional tubes in the inner layer of each wall run perpendicular to the beam axis and thus provide spatial information for the polar angle $\theta$. The tubes in the outer three layers run parallel to the beam axix and thus provide opatial information for the azimuthal angle $\phi$.

Table 2.2. Hadron absorber thickness in the muon system for normal incidence. The nuclear interaction length for iron is $132 \mathrm{~g} / \mathrm{cm}^{2}$

\begin{tabular}{|c|c|c|c|c|c|}
\hline \multirow[b]{2}{*}{ Layer } & \multicolumn{4}{|c|}{ Absorber Thickness $\left(\mathrm{g} / \mathrm{cm}^{2}\right)$} & \multirow{2}{*}{$\begin{array}{l}\text { Interaction } \\
\text { Lengtha } \\
\text { Average }\end{array}$} \\
\hline & East Wall & Top Wall & West Wall & Bottom Wall & \\
\hline Magnet and $\mathrm{LA}$ & & & & & 1.2 \\
\hline 1 & 182 & 182 & 182 & 182 & 1.4 \\
\hline 2 & 184 & 184 & 184 & 184 & 1.4 \\
\hline 3 & 244 & 239 & 244 & 244 & 1.8 \\
\hline 4 & 196 & 184 & 196 & 244 & 1.6 \\
\hline Total & 806 & 789 & 806 & 854 & 7.4 \\
\hline
\end{tabular}

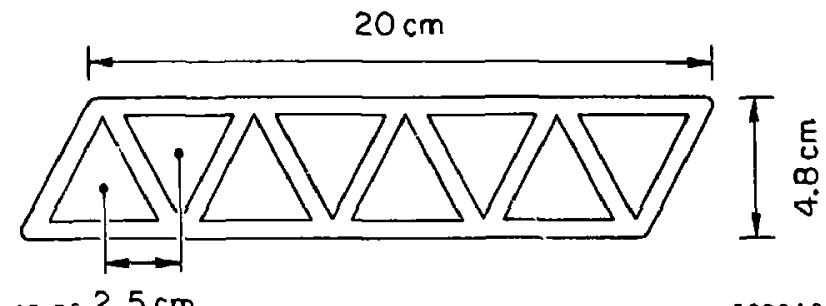

$12-852.5 \mathrm{~cm}$

$5290 A 2$

Each layer of proportional tubes consists of extruded aluminum modules cach comprising 8 triangular tubes as shown in Figure 2.6. The center-to-center spacing of the tubes is $2.5 \mathrm{~cm}$, a distance which is well matched to the typical extrapolation error from the drift chamber tracking error and multiple Coulomb scattering.

Figure 2.6. Cross sectional view of a muon proportional tube module. 


\subsection{ENDGAP CALORIMETERS}

The endcap calorimeters cover the polar angular region between $\approx 15^{\circ}$ and $\approx 40^{\circ}$. Each endcap consists of two layers of proportional chambers each preceded by 2.3 radiation lengths of lead. The encrgy resolution for photons and electrons is $\sigma_{E} / E \approx 0.50 E^{-1 / 2}$ where $E$ is the energy in GeV.

\subsection{Small angle Calorimeter}

The small angle tagging (SAT) system covers the angular region betwecn 21 and $82 \mathrm{mrad}$ with respect to the beamline. The system is designed to identify the scattered electrons from low momentum transfer Bhabla scattering and two photon processes. The measurement of the small angle Bhabba cross section provides a delermination of the luminosity to $\pm 5 \%$.

Each SAT counter system consists of three sets of planar drift chambers, three layers of acceptance defining scinti\}lation counters, and a shower counter made up of eighteen layers of $1 / 4$ inch thick lead and $1 / 2$ inch thick plastic scintillator. The energy resolution of the shower counters is $\sigma_{E} / E \approx 0.16 E^{-1 / 2}$.

\subsection{EVENT TRIGGER}

The decision about whether or not to record a particular event on magnelic tape is made by a two level logic system. The first decision process, called the primary trigger, is completed in $\approx J \mu s$. Since the time between beam crossings ot PEP is $2.4 \mu s$, there is no deadtime associated with the primary trigger. If an event satisfies the primary trigger, a signal is sent to the detector electronics to prevent data collection from subsequent beam crossings and the event is tested by a secondary trigger which takes about $30 \mu \mathrm{s}$ to make a decision. This resulto in a deadtime of $3 \%$ per $k H z$ of primary rate.

The primary trigger logic is atisfied by any one of the following conditions,

- Charged particle primary trigger: A coincidence of a beam crossing signal, a drift chamber signal from at least 3 of the 7 inner vertex chamber la jers and at least 6 of the cuter drift chamber layers spread out in radius, and at least one TOF counter signal.

- Neutral primary trigger: At least two calorimeter modules with at least $\approx 1 \mathrm{GeV}$ of energy deposited in the front half of a liquid argon module or at least $\approx 3 G e V$ deposited in an endcap module.

or

At least $\approx 4 \mathrm{GeV}$ of energy deposited in the front half of all eight $\mathrm{LA}$ modules summed together or in both endcaps.

- SAT Bhabha primary trigger: Collinear electron-positron candidate found by the SAT electronics.

For the secondary trigger, 24 microprocessors work in parallel to identify charged track candidates, called hardware tracks, in the drift chamber. The secondary trigger logic is satisfied by any one of the following conditions.

- Charged track secondary trigger: At least 2 hasdware tracks.

- Neutral secondary trigger: Same es neutral primary trigger. 
Only one calorimeter module over throshold and only one hardware track.

- SAT-Bhabha secondary trigger: One of every sixteen SAT-Bhabha primary triggers.

The primary trigger rate was typically less than $1 \mathrm{kHz}$ resulting in less than $3 \%$ deadtime from the secondary trigger. The secondary trigger rate was typically 1 to $2 \mathrm{~Hz}$.

\section{Particle Identification}

Particle identification utilizes the fact that electrons, muons and hadrons each intetact with matter in a characteristic way. Electrons and photons interact predominantly through bremsstrahlung and pair production with a scale set by the radiation length, $X_{0}$, which is related to the mean path length of an electron in the material. Bremsstrahlung is suppressed for the muon and for hadrons because of their large mass. Hadrons interact with the nuclei of the material through the strong interaction with a scale set by the nuclear absorption length, $\lambda$. A material which has a small radiation length and a large nuclear absorption length is used to discriminate clectrons from pions. Since the muon is a lepton it does not participate in the strong interaction. Therefore, the muon loses much less energy in an absorber material than electrons or hadrons and can be identified by its ability to penetrate.

In this analysis, information from the drift chambers, the LA calorimeter, and the muon system is used to identify charged tracks as electrons, muons or hadrons. Information from the LA and end cap calorimeters are used to ident:fy photons. In the event selection, very efficient muon and clectron rejection algorithms are needed, while in the measurement of the branching fractions, algorithms which discriminate between electrons, muons and hadrons in a manner which is well simulated by the Monte Carlo are needed. Both types of particle identification algorithms are discussed in this chapter and the efficiencies for these algorithms are discussed in detail in later bections of the thesis. 


\subsection{MUON IDENTIFICATION}

Muon identification utilizes the fact that muons have a very low cross-section for interacting with matter. The primary means of energy loss experienced by the muon in passing through material is due to ionization of atoms and molecules in the material. For iron, this relatively uniform energy loss is about $200 \mathrm{MeV}$ per interaction length.

The muon identification system is described in Chapter 2 . It consists of 4 layers of iron hadron absorber, each followed by a layer of proportional tubes. To identify muons, each track in the drift chamber is projected into the muon system. At each level a search for proportional tube signals is made in a region around the projected track. The search region is set to three times the rms extrapolation error. The extrapolation error is determined from the expected multiple scattering in the coil LA system and iron hadron absorber preceding the layer of proportional Lubes, and the expected tracking error, added in quadrature.

The mean squared extrapolation error due to multiple Coulomb scattering for the coordinate of interest for a partic $\cdot$ lar level is

$$
\sigma_{z}^{2}=\frac{1}{2} \sum_{i} \theta_{i}^{2}\left(\frac{t_{1}^{2}}{3}+t_{i} d_{i}+d_{i}^{2}\right)
$$

where $t_{i}$ is the absorber thickness for detector element $i, d_{i}$ is the drift distance following element $i$, and $\theta_{i}$ is the rms scattering angle in the Gaussian approximation, given by

$$
\theta_{i}=\frac{21 \mathrm{MeV} / \mathrm{c}}{p(M e V / c) \beta^{\prime}}\left(\frac{t_{i}}{L_{\text {rad }}}\right)^{1 / 2}
$$

$p$ and $\beta$ are the momentum and velocity of the incident particie and $L_{\text {rad }}$ is the radiation length of the absorber. This results in typical multiple Bcattering errors of

$$
\sigma_{x} \approx \frac{x}{p(G e V / c)}
$$

where $\boldsymbol{I}$ is about $10 \mathrm{~cm}$ for the first level and $20 \mathrm{~cm}$ for the fourth level.

The tracking extrapolation error depends on several factors. It is larger for the modules on the sides of the detector than for those on the top and bottom because the modules on the sides are further from the origin. The error is larger for the first layer than for the other three layers because the proportional tubes in the first layer are oriented perpendicular to the beam and so measure the track crossing point in $z$, the coordinate which is least well measured by the drift chamber. The extrapolation errors due to tracking used in the muon identification algorithro are shown in Table 3.1.

Table 3.1. Extrapolation error (in $\mathrm{cm}$ ) due to tracking by layer and position of module.

\begin{tabular}{|c|c|c|c|c|}
\hline Layer & East & Top & West & Bottom \\
\hline 1 & 3.0 & 2.0 & 3.0 & 2.0 \\
2 & 2.0 & 1.5 & 2.0 & 1.5 \\
3 & 2.3 & 1.8 & 2.3 & 1.8 \\
4 & 2.6 & 2.1 & 2.6 & 2.1 \\
\hline
\end{tabular}

3.1.1 $\mu / \pi$ Discrimination

In this analysis, muons are defined to be tracks which are expected to penetrate 
all four layers of absorber material il they are muons and which have a signal within the search region in all four layers of proportional tubes. The first condition means that, based on the charged particle tracking in the drift chamber, a muon with the particle's momentum would have sufficient energy and would be travelling in the correct direction to penetrate all four layers of absorber. Due to the uniform energy loss described above, muons with momentum less than about $2 \mathrm{GeV} / \mathrm{c}$ are not expected to penetrate the absorber material to the fourth layer of proportional tubes. Hence, $\mu / \pi$ discrimination is only attempted for particles with momenta greater than $2 \mathrm{GeV} / \mathrm{c}$.

\subsubsection{Muon Rejection}

A track is classified as not being associated with a muon if it is expected to penetrate at least three layers of absorber if it is a muon, the three $\sigma$ search region lies within the fiducial volume for at least three layers, and it has no associated hits or has associated hits in the first and/or last layer only. A hit in the first layer only, for a track with enough momentum to penctrate at least three layers if it is a muon, is often due to hadronic punchthrough. Isolated hits in the last layer are often noise hits from synchrotron radiation reflecting off the walls in the detector hall.

These muon rejection criteria are stricter than the $\mu / \pi$ discrimination criteria in two ways. For muon rejection, the track trajectory passes through each layer of the muon system at least three $\sigma$ from the edge, where $\sigma$ is the rms extrapolation error; for $\mu / \pi$ discrimination, the trajectory passes through any part of each of the lour layers. For muon rejection, the track has at most an associated hit in the first and last layer; for $\mu / \pi$ discrimination, the track is not classified as a muon if it has associated hits in less than four layers.

\subsection{ELEGTRON IDENTIFICATION}

Electrons are identified by observing the energy deposition in the 'aad - liquid argon calorimeters The ratio of the nuclear absorption length, $\lambda$, to the radiation length, $X_{0}$, of a material is proportional to $Z^{2} / A^{3 / 4}$. Therefore, $\lambda / X_{0}$ is large for high $Z$ materials such as lead. The nucleat absorption length is $9.8 \mathrm{~cm}$ for lead while the radiation length is only $0.56 \mathrm{~cm}$. Hence electrons are much more likely than hadrons to deposit their energy in the 14 radiation lengths of material in the LA calorimeter. The LA calorimeter is described in Chapter 2. Recall tr: esch module consists of 18 layers of lead strips which are ganged to form 6 readout layers in depth.

Information from the $\mathrm{LA}$ calorimeter is used for both electron rejection and $e / \pi$ discrimination at different stages in this analvsis. Therefore, electron identification is performed using two different methods. One method checks that the amount of energy found in various layers of the LA calorimeter (without sharing) is greater than the minimum expected for an electron shower. This method is used for $e / \pi$ discrimination. The second method associates energy in the LA calorimeter with a track. If the associated energy is greater than half of the momentum of the track measured with the drift chamber, the track is classified as an electron. This method has an extremely high efficiency for electron rejection. Both methods are described 
in detail in the following sections.

\subsection{1 e/ $\pi$ Discrimination}

The algorithm for $e / \pi$ discrimination tests whether or not the longitudinal distribution of energy in the shower is consistent with that for an electron. A variable, called TEST1, is calculated for all charged tracks which project in to the fiducial volume of the $\mathrm{LA}$ calorimeter. TEST1 is the minimum of the following:

- $\frac{E_{F_{1}}+E_{F_{2}}}{\alpha_{F} \times p}$

- $\frac{E_{T_{1}}}{a_{T} \times p}$

- $\frac{E_{U}}{\alpha U \times p}$

- EFront

where F1, F2, T1 and $U$ refer to the $L A$ readout layers as illustrated in Figure 2.5. $E_{i}$ is the energy deposited within a narrow region (described below) around the projected drift chamber track in layer $i, p$ is the momentum as measured with the drift chamber, and $\alpha_{i}$ is the minimum fraction of an electron's encrgy that is expected to be deposited in layer $i$. Front refers to the sum over layers F1, F2, T1 and $U$ which comprise the first $\approx 7$ radiation lengths of the calorimeter. The values of $\alpha_{i}$ used in the calculation of TESTI are given in Table 3.2 .

The search region around the projected drift chamber track is chosen to be small to reduce the probability of misidentifying a pion as an electron by assigning to it energy deposited by nearby electrons or photons. The width of the search
Table 3.2. Minimum fraction of the total energy typically deposited by an electron within a narraw region of the extrapolated drift chamber track in various layers of the IA calorimeter.

\begin{tabular}{|c|c|c|}
\hline & \multicolumn{2}{|c|}{ Momentum Range } \\
\hline Layer & $\leq 4 \mathrm{GeV} / \mathrm{c}$ & $>4 \mathrm{GeV} / \mathrm{c}$ \\
\hline $\mathrm{F} 1+\mathrm{F} 2$ & 0.14 & 0.14 \\
$\mathrm{~T} 1$ & 0.10 & 0.10 \\
$\mathrm{U}$ & 0.10 & 0.10 \\
Front & 0.50 & 0.40 \\
\hline
\end{tabular}

region, warch, is calculated according to the following formula:

$$
w_{\text {search }}=w_{\text {shower }}+w_{\text {garg }} \times|\tan \delta|
$$

where $w_{\text {shower }}$ is the typical width of an electromagnetic shower $(\approx 3 \mathrm{~cm})$, and the second term reflects the additional width arising from the separation $(\approx 4-7 \mathrm{~cm})$ of the frant and back of a ganged layer, $w_{\text {gang. }} \delta$ is the angle between the track projection and the normal to the layer measured in the plane which contains the strip coordinate and the normal. Table 3.3 shows typical values of $w_{\text {shower }}$ and $w_{\text {gang }}$ used in the algorithm, in units of strip center-to-center apacing. Recall that the center-to-center spacing is $3.8 \mathrm{~cm}$ for $F$ and $T$ strips and $5.4 \mathrm{~cm}$ for $U$ strips. If the cenics of a strip lies within the search region, then the energy from that strip is associated with the projected track.

The distribution of TEST1 for electrons and pions in the data is shown in Figure 3.1 for particles with momentum between 5 and $10 \mathrm{GeV} / c$. The pions are 
Table 3.3. Typical values of $w_{\text {shower }}$ and $w_{\text {gang }}$ used in the calculation of the search region in units of strip center-to-center spacing.

\begin{tabular}{|c|c|c|}
\hline & \multicolumn{2}{|c|}{ width in units of strip center-to-center spacing } \\
\hline Layer & $w_{\text {shower }}$ & $w_{\text {gang }}$ \\
\hline F1, F2 & 0.75 & 0.9 \\
T1 & 0.75 & 1.5 \\
U & 0.70 & 1.5 \\
\hline
\end{tabular}

from decays of the tau to three charged particles and the electrons are from radiative Bhabha events. A value of TEST1 $=0.85$ provides about equal efficiency for electron and pion identification. Discrimination of electrons from other particles is only attempted if the drift chamber track projects to within 2.5 strips of the edge in the

F1 layer and 1.5 atrips of the edge in the F2 and T1 layers.

\subsubsection{Electron Rejection}

The algorithm used for efficient elcctron rejection makes use of the fact that electrons are very likely to deposit all of their energy in the LA calorimeter. Energy deposited in the LA calorimeter is associated with charged tracks in the following way. The charged track is projected from the drift chamber into the calorimeter. For each layer, a search is made for the strip closest to the projected track which has a signal above a certain threshold. This strip and adjacent strips are studied to form a group of strips to be associated with the track. The number of strips in a group is limited to four unless the energy of the sum of the selected strips is greater than another threshold, in which case the maximum allowed number of

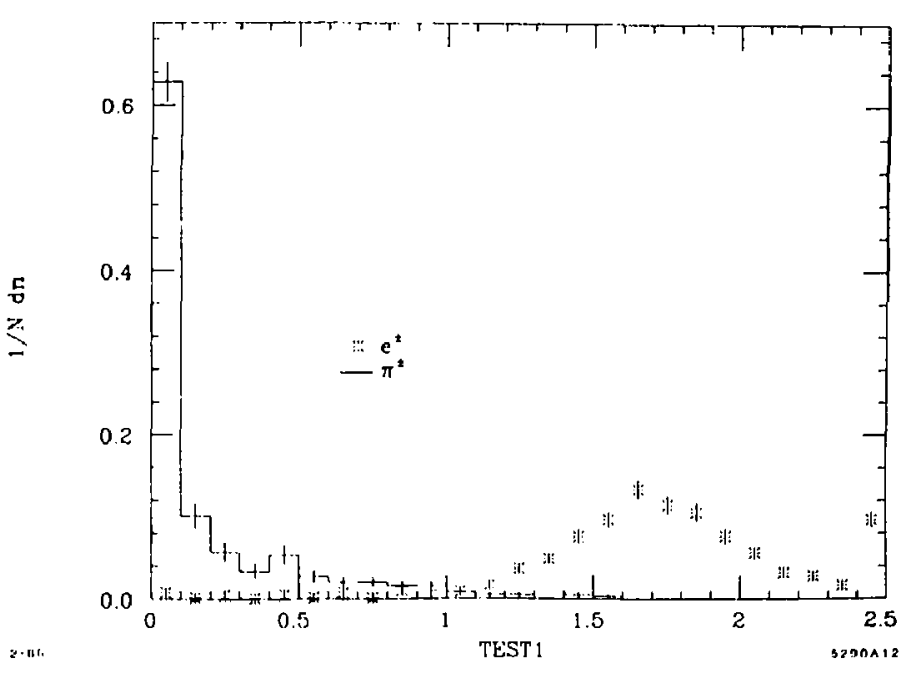

Figure 3.1. TEST1 distribution for electrons and pions in PEP data. The momenta of the elcctrons and pions is between 5 and $10 \mathrm{GeV} / \mathrm{c}$. 
strips is six. In forming groups, strips with less than two times the noise level (as measured during calibration) are ignored. Only contiguous strips with signals are included in a group (dead channels are ignored). The total pulse height for each layer is converted to energy using calibration constants determined from Bhabha events. The energies of the six readout layers are summed and corrected for energy loss in the magnet coil preceding the calorimeter and leakage out the back of the coil.

Figure 3.2 shows the detection efficiency of the LA calorimeter for electrons from collinear $e^{+} e^{-} \rightarrow e^{+} e^{-}$events as a function of the distance from the center of the modules along the direction of the beam $(z)$, and as a function of the azimuthal angle from the point between two modules $(\phi)$. For electron rejection, the active volume of the $\mathrm{LA}$ calorimeter is defined to be the area less than $1.75 \mathrm{~m}$ from the module center in $z$ and less than 0.345 radians relative to the module center in $\phi_{1}$ as indicated by the arrows in the figure. A track is only considered for electron rejection if both the projected drift chamber track and the center of the shower cluster in the trigger gap are contained within the active volume.

As mentioned above, efectrons are very likely to deposit all of their energy in the $L A$ calorimeter while hadrons are likcly to only deposit a small amount of their energy due to uniform ionization along the track. Consequently, the simple ratio $E / p$, where $E$ is the energy in the LA calorimeter associated with the track and $p$ is the momemtum of the track, can be used to identify a charged particle as an electron or a pion. The probability that $E / p$ is greater than 0.5 is close to $100 \%$
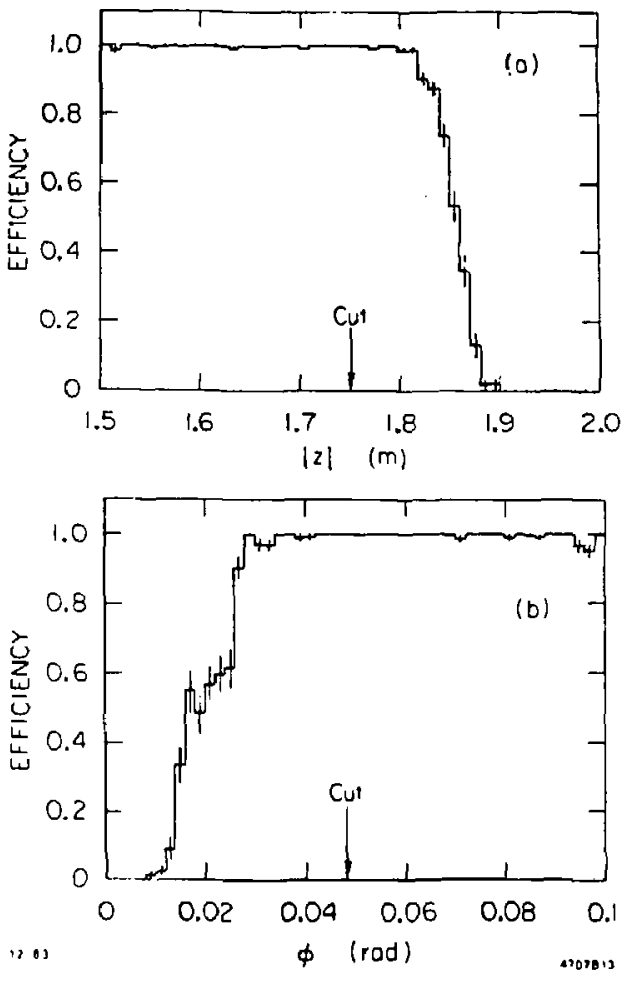

Figure 3.2. Detection efficiency of the LA calorimeter for electrons from collinear $e^{+} e^{-} \rightarrow e^{+} e^{-}$events as a function of the distance from the center of the modules along the direction of the beam $(z)$ and as a function of the azimuthal angle from the point between two modules $(\phi)$. The arrows indicate the edge of the active volutne used for electron rejection. 
for electrons with momenta above $1 \mathrm{GeV} / \mathrm{c}$ which project into the active volume of the LA system. The probability that $E / p$ is less than 0.5 is about $90 \%$ for pions. Therelore the criteria that $E / p$ be less than 0.5 provides excellent electron rejection while misidentifying about $10 \%$ of pions as electrons.

\subsection{PHOTON IDENTIFICATION}

Photons are identified using an algorithm which searches for clusters of energy in the $\mathbf{L A}$ calorimeter. First, a search is made in each layer for contiguous strips with deposited energy. The position of the centroid of this group of strips is calculated. Photon candidates are constructed from opatial coincidences of groups from the different layers, with at least one group associated with each of the threc strip orientations. If a group of strips in a layer is associated with two photons, the energy of the group is divided between the two photrns according to the ratio of the unshared energy in the showers. Any photon which shares groups in layers of two or more different orientations is discarded and the shared energy is reassigned to the candidates which shared the groups.

Spurious candidates which are not associated with real photons can result from the coincidence of noise fluctuations and real deposited energy. Also, hadronic interactions in the LA calorimeter can result in energy depostion which is sometimes far from the charged track. These two effects result in fake photon candidates with an energy spectrum peaked at very low values. Figure 3.3 shows the energy spectrum for photon candidates in the decays $T^{-} \rightarrow \nu_{T} \pi^{-}$and $T^{-} \rightarrow \nu_{T} \pi^{-} \pi^{+} \pi^{-}$ from a Monte Carlo simulation. The probability of finding a spurious photon candidate with any energy is about $23 \%$ and $76 \%$ for the decay to one charged pion and three charged pions, respectively. The figure shows that the energy of a fake photon candidate is usually less than about $0.65 \mathrm{GeV}$. In this analysis, photon candidates are defined to be those neutral energy clusters with more than about $0.5 \mathrm{GeV} / \mathrm{c}$ associated energy. This cutof value is varind in check the sensitivity of the results to the number of spurious photons.

In an independent analysis of three prong decays of the tau using data from the Mark II detector, ${ }^{40} \mathrm{t}$ was shown that with an energy cutofl of about $(0.5-0.8) \mathrm{GeV}$ the probability of finding a fake photon in the data is approximately $50 \%$ higher than in the Monte Carlo. This correction is applied when using the number of neutrals Found in a candidate tau decay to predict the branching fractions. The effects of the uncertainty in this correction are dise ussed in the section on systematic errors in Chapter 7

\section{$3.4 e^{+} e^{-}$PAIH-FINDING ALgORITHM}

In the event selection, it is necessary to remove some events with pairs of charged tracks resulting from the conversion of a photon into an $e^{+} e^{-}$pair when passing through material. The pair-finding algorithm is based on the fact that the opening angle between the two tracks is small in the laboratory frame. The opening angle is more accurately measured in the plane perpendicular to the beams (the $x-y$ plane) than in the plane parallel to the beams. Therefore, the pair-finding criteria are applied separately in the two planes. This is better than using the invariant mass of the two charged particles as the criteria for identifying pairs since the mass 


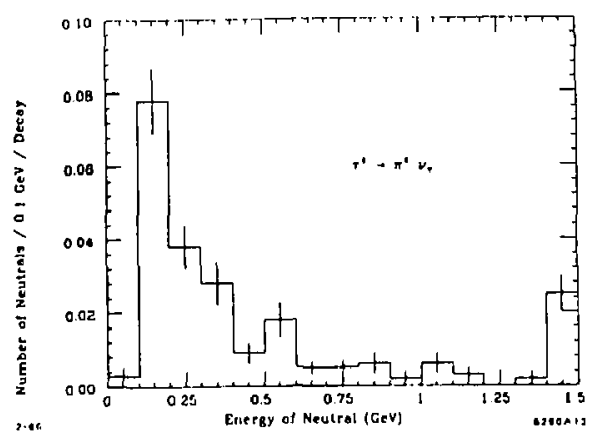

resolution is degraded by the poor polar angle measurements.

For each pair of oppositely charged tracks, the following parameters are calculated: the distance between the two tracks in the $x-y$ plane where the two circle fits to the tracks are tangential $\left(\Delta_{x y}\right)$; the distance between the origin and the above point in the $x-y$ plane $\left(R_{x y}\right)$; the distance between the two tracks in the $z$ direction at the distance of closest approach to the origin $\left(\Delta z_{0}\right)$; and the difference in the polar angle of the two tracks $(\Delta \theta)$. These parameters are illustrated in Figure 3.4. $\Delta_{x y}$ is related to the opening angle in the $x-y$ plane and $R_{x y}$ is close to the point of conversion for $e^{+} e^{-}$pairs. $\Delta z_{0}$ is a measure of how well the track was reconstructed in the $z$ direction and $\Delta \theta$ is the opening angle in the plane parallel to the beams.

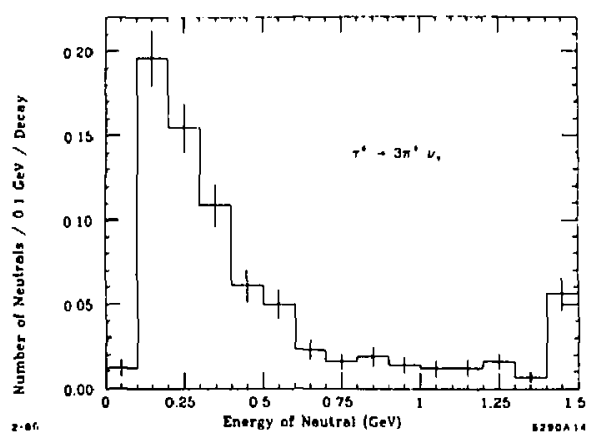

The pair of tracks is classified as an $e^{+} e^{-}$pair from the conversion of a photon if $\left|\Delta_{x y}\right|<1.5 \mathrm{~cm}$ and $R_{x y}>-10 \mathrm{~cm}$. In addition, if $\Delta z<10 \mathrm{~cm}$, the event is classified as an $e^{+} e^{-}$pair only if $\Delta \theta<0.1$ radians. Besides eliminating $e^{+} e^{-}$pairs from photon conversions, the above criteria will eliminate some pairs from $\pi^{0}$ 's which undergo Dalitz decay $\left(\pi^{0} \rightarrow y e^{+} e^{-}\right)$. 


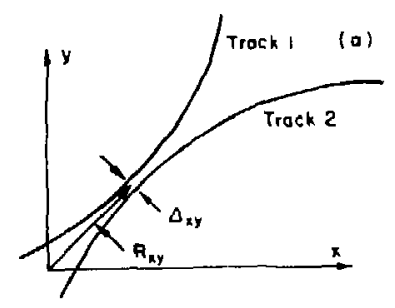

\section{Event Selection}

4.1 GeNeral PHILOSOPhy

The goal of this analysis is to sirrultaneously measure the branching fractions for all decay modes of the tau by performing a maximum likelihood fit to an unbiased sample of tau decays. In this chapter, the selection of even ts for the unbiased sample is described.

Since tau leptons are a]way's produced in oppositely charged pairs at PEP

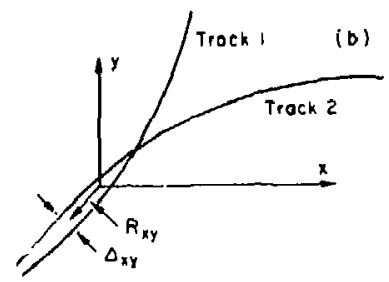
and the decay products of the two leptons are easily separated, one can select an unbiased sample of tau decays by using the decay products of cne tau in the pair to tag the event and then including the opposite tau in the sample. Of course, if the decay products of earh tau pass the criteria for a tag, then both members of the tau pair are included in the fnal sample of tagged tau decays. For this procedure

- to work, the tag must correspond to a decay mode of the tau which is distinctive of the tau and not of other processes which occur at PEP. This is necessary to ensure little background in the sample. To maximize the number of candidates in the final sample, the tag should correspond to a decay mode with as large a branching fraction as possible.

Two different tags ate used in this analysis resulting in two distinct samples of tau decays. Basically, one tag corresponris to a single charged hadron and is reforred to as the 1 prong tag; the other taly corresponds to three charged particles and is referred to as the 3 prong tag. In both tags, a limited number of neutral energy clusters are allowed. These tags are described in detail below. The tav- 
candidate samples corresponding to the two tags have different potential sources of background and slightly different systematic errors. Therefore, they provide an important cross check on the final results.

The leptonic decay modes account for $\approx 40 \%$ of the total decay width of the tau. Since this is such a large portion, a leptonic tag was also attempted. Unfcrtunately, the background from QED events of the type $e^{+} e^{-} \rightarrow e^{+} e^{-}(\gamma)$ and $\mu^{+} \mu^{-} \rightarrow \mu^{+} \mu^{-}(\gamma)$ is so high that a leptonic tag is not useful.

\subsection{OVERall CUTS}

There are various criteria which the overall event must meet to make it into the final sample. First, the event must pass the trigger requirements. The trigger decisions are based on logic signals produced by trigger hardware that is difficult to simulate accurately in the Monte Carlo. Consequently, I impose a condition in software which is slightly more restrictive than the hardware trigger and which is simulated well in the Monte Carlo. Only events with at least 2 tracks with polar angle $\theta$ satisfying $|\cos \theta| \leq 0.7$ are accepted.

Various cuts are also made to reduce the amount of data written to the final summary tapes. The specific criteria an event must meet are:

- $\left|z_{\mathrm{u}}\right| \leq 20 \mathrm{~cm}$ where $z_{\mathrm{y}}$ is the vertex position along the beam direction relative to the beam collision point;

- $\geq 2$ charged tracks;

- $E_{\text {ch }}>\frac{E_{\text {beam }}}{4} \approx 3.6 \mathrm{GeV}$ where $E_{\text {ch }}$ is the total charged energy in the event;

- $E_{t o t}>\frac{E_{t e a m}}{2} \approx 7 \mathrm{GeV}$ where $E_{t o t}$ is the total charged plus neutral energy in the cvent.

However, the following events are exempt from the above cuts:

- any event consistent with the QED production of $e^{+} e^{-}, \mu^{+} \mu^{-}$, or $\Upsilon \Upsilon$ pairs;

- any event containing an electron* with momentum greater than $1.25 \mathrm{GeV} / \mathrm{c}$;

- any event containing a track with associated hits in at least three levels of the muon system.

A Monte Carlo simulation shows that the probability of a one prong decay of the tau meeting the above criteria is about $42 \%$, independent of the decay mode. The probability for a three prong decay is about $51 \%$. This increase of about $20 \%$ for three prong decays is due to the larger number of charged tracks available to fulfill the trigger requirements and to the larger amount of visible energy in the event.

\subsection{THE 1 P'RONG TAG}

4.3.1 Selertion C'titcria

bach event is divided into two hemispheres by the plane perpendicular to the chirust axis of the event. All charged tracks and neutral energy clusters with greater tinan $500 \mathrm{M} \mathrm{EV}$ deposited in the LA or end cap calorimeters are used in the calculation of the thrust axis. The 1 prong tag corresponds to decay products in one hemisphere of the event with the following prope-ties.

- The hemisphere contains exactly one ch.argad track which projects into the

- Here, an electron is defined to be any track with time-of-flight or LA calorimeter information consistent with an electron. 

altive volutue of the liquid Argon calorimeter and the muon system, is identified as definitely not being an electron or a muon, and has momentum below $10 \mathrm{GeV} / \mathrm{c}$ and above $2 \mathrm{GeV} / \mathrm{c}$. The total visible energy in the hemisphere (charged*. plus neutrai) is less than $12 \mathrm{GeV}$. These criteria seject QED events of the type $e^{+} e^{* *} \rightarrow e^{+} e^{-}(\gamma), e^{+} e^{-} \rightarrow \mu^{+} \mu^{-}(\gamma)$, $e^{+} e^{-} \rightarrow e^{+} e^{-} e^{+} e^{-}$, and $e^{+} e^{-} \rightarrow e^{+} e^{-} \mu^{+} \mu^{-}$.

- The hemisphere contains three or fewer neutral energy clusters. The invariant mass of the charged particle (assumed to be a $\pi$ ) plus neutrals is less than $1.5 \mathrm{GeV} / \mathrm{c}^{2}$. These criteria reject QCD events.

The major decay modes of the tau which have the above properties are $\tau^{-} \rightarrow \nu_{\tau} P^{-}, \tau^{-} \rightarrow \nu_{\tau} \pi^{-}$and $\tau^{-} \rightarrow \nu_{r} \pi^{*} 2 \pi^{0}$. Recall that the branching fractions

if for these modes are about $22 \%, 10 \%$ and $8 \%$, sespectively. They contribute - approximately $48 \%, 28 \%$ and $14 \%$, respectively, to the tag assuming the above branching fractions.

At this point, a cut is made on the invariant mass of the charged and neutral particles (assumed to be pions and photons, respectively) in the tau-deray candidate opposite the 1 prong tag. If the mass is greater than $2.5 \mathrm{GeV} / \mathrm{c}^{2}$, the candidate

is rejected. The number of candidates rejected is used to estimate the hadronic background (see Chapter 6 ).

* The maximum of the mumentum measured by the drift chamber and the energy in the LA calorimeter associated with the track is used for the energy of the charged track.

\subsubsection{Efinciency}

According to Monte Carlo simulations of tau decays and the detector response, the probability of ary tau decay making it into the sample tagged with the 1 prong tag is $3.9 \%$. The major factors which contribute to the efficiency are the overall event cuts described above $(\epsilon \approx 43 \%)$, the branching fraction to one charged hadron $(\epsilon \approx 40 \%)$, the requirement that the charged track in the tag pass through the active volume of the $L A$ calorimeter and the muon system $(\epsilon \approx 50 \%)$, and the momentum cuts of 2 and $10 \mathrm{GeV} / \mathrm{c}$ for the tag $(\epsilon \approx 65 \%)$.

In the Mark II data sample, the number of jets which pass all of the criteria for the 1 prong tag is 1627 . Since the total integrated luminosity in the data sample is $207 \mathrm{pb}^{\cdots 1}$ and the cross section for tau-pair production (including second order QED corrections) is $136 \mathrm{pb}$ at $\sqrt{s}=29 \mathrm{GeV}$, the total number of tau pairs in the data satngle is about 28000 . Therefore, the efficiency for a tau decay to pass the citeria for the 1 prong tag is $162 \pi^{\prime}(2 \times 28000) \approx 2.9 \%$, assuming little background. This does not agree well with the efificiency predicted by the Monte Carlo. There are several reasons for the discrepancy. First, the lepton rejection criteria are very strict. Therefore, noise in the muon system or the LA calorineter can cruse a hadron to fail the rejection criteria. If the noise is not accurately simulated in the Monte Carte, the efficiency will not arree with the data. Secondly, the cut on the number of neutral enerzy clusters in the hemisphere is sensitive to the number of

- The diurnher in parentheses is the probability for passing the cut if the event passed all of the earlier cuts. 
fake photon candidates found. It is known that the Moste Cajlo does not produce as many spurious .1eutral energy clusters in the LA calorimeter as are found in the - data. Also the efficienc' predicted by the Monte Curlo depends on the branching fractions used.

Since the absolute efficiency for a tau to pass the 1 prong tag does not enter into the calculation of the branching fractions, it does not matter that the efficiency predicted by the Monte Carlo disagrees with the data. This points out one of the ndvantages of this type of analysis. The strict cuts which are sensitive to effects that are difficult to simulate accurately in the Monte Carlo, but which are necessary to reduce the background contribution to the sample, are applied to the tag side of the event. Then less severe criteria which are relatively insensitive to the Monte Carlo simulation are used to identify the decay modes in the tau-candidate sample. Even though the measurement of the branching fractions does not depend on the absolute probability for any decay mode to be included in the sample, it does depend on the relative probability for the different decay modes. If each decay mode is equally likely to be included in the sample, the sample is unbiased. This is discussed ir more detail in Chapter 5 .

\subsection{THE 3 Prong TAG \\ 4.4.1 Selection Criteria}

The 3 prong tag corresponds to decay products in one hemisphere of the event with the following properties.

- The hemisphere contains exactly three charged particles with net charge \pm 1 .
- The total visible energy in the hemisphere (charged* plus neutral) is les: than $14 \mathrm{GeV}$. This provides some rejection against radiative QED events.

- No oppositely charged pairs of tracks in the hemisphere satisfy the pair finding algorithm described in Chapter 3. This cut rejects events corres ponding to radiative QED production of $e^{+} e^{-}$and $\mu^{+} \mu^{-}$pairs in which the photon converts to an $e^{+} e^{-}$pair.

- The hemisphere contains two or fewe neutral energy clusters. The invariant mass of the charged particles (assumed to be $\pi$ 's) plus neutrals is less than $1.7 \mathrm{GeV} / \mathrm{e}^{2}$. These crlliria reject QCD events.

- Each charged track (assumed to be $\mathrm{a} \pi$ ) and neutral track in the hemisphere is boosted along the thrust axis of the event into the rest irame of a $T$ moving with the beam energy in the laboratory frame. The magnitude of the vector sum of the particle momenta in this frame, which is called $p^{*}$, represents the magnitude of the momentum carried off by the neutrino in real tau decays assuming no initial or final state radiation. Events are accepted if $p^{*}$ is less than $750 \mathrm{MeV} / \mathrm{c}$. For $\tau^{-} \rightarrow \nu_{\tau} \pi^{-} \pi^{+} \pi^{-}\left(\pi^{0}\right)$, Monte Garlo simulations show that for about $84 \%$ of the decays $p^{*}$ is less than $750 \mathrm{MeV} / \mathrm{c}$. On the other hand, only about $14 \%$ of hadronic jets with 3 charged particles in the data have $p^{*}$ less than $750 \mathrm{MeV} / \mathrm{c}$. Jets produced via the two photon interaction

* The maximum of the momentum measured by the drift chamber and the energy in the LA calorimeter associated with the track is used for the energy of each charged track. 
are also very unlikely to satisfy this condition.

The major decay modes of the $\tau$ which have the above properties are $\tau^{-}-$ $\nu_{r} \pi^{-} \pi^{+} \pi^{-}$and $\tau^{-} \rightarrow \nu_{r} \pi^{-} \pi^{+} \pi^{-} \pi^{0}$. Recall that the branching fractions for these modes are about $8 \%$ and $5 \%$, respectively. They contribute approximately $60 \%$ and $40 \%$, respectively, to the tag assuming the above branching fractions.

As in the case of the 1 f $6 \mathrm{~g}$ ag, a cut is made on the invariant mass of the charged and neutral parti $y=$ (ass: :il $\therefore$ to be pions end photons, respectively) in the tau-decay candidate oppor $: \cdots, \cdots$, prong tag. If the mass is greater than $2.5 \mathrm{GeV} / \mathrm{c}^{2}$, the candidate is rejected. The number of candidates rejected is used to verify the hadronic background estimated using the $p^{\bullet}$ cut (see Chapter 6 ).

\subsubsection{Efficiency}

According to Monte Carlo simulations, the probability of any tau decay making it into the sample tagged with the 3 prong tag is about $3.5 \%$. The major factors which contribute to the efficiency are the general event cuts described above $(\epsilon \approx 43 \%)$, the branching fraction to three charged particles $(\epsilon \approx 13 \%)$, the total energy cut on the $\operatorname{tag}(t \approx 92 \%)$, the $e^{+} \epsilon^{-}$pair cuts $(\epsilon \approx B 1 \%)$, and the $p^{*}$ cut on the tag $(\epsilon \approx 84 \%)$.

In the Mark II data sample, the number of jets which pass all of the criteria for the 3 prong tag is 1475 . Therefore, the efficiency for a tau decay to pass the criteria for the 3 prong tag is $1475 /(2 \times 28000) \approx 2.6 \%$, assuming little background. This does not agree well with the efficiency predicted by the Monte Carlo. Again, this does not matter, since this efficiency does not enter into the calculation of the 
5. Unfolding the Branching Fractions

\subsection{OBSERVED TOPOLOGIES}

To determine the branching fractions, the tagged tau decays in each sample are divided into subgroups by charged and neutral particle multiplicity, and particle type where possible. The categories are chosen so that for each decay mode there is a category for which that decay snode is the major contributor with little contribution from other decay modes. Also, the criteria for the categories are chosen to be those that are well modelled by the Monte Carlo simulation program so that the systematic errors are kept to a minimum.

First, the tagged tau decays are divided into subgroups according to the number of charged particles in the hemisphere. If there is one charged particle, the decay is further classified according to the identification of that particle. Recall from Chapter 3 that muon identification is possible for tracks with momentum above $2 \mathrm{GeV} / \mathrm{c}$ and electron identification is possible for tracks with momentum above $1 \mathrm{GeV} / \mathrm{c}$. In the remainder of this section, I will define a track to be a pion if it is not a muon and not an electron according to the $\mu / \pi$ and $e / \pi$ discrimination algorithms. Therefore, pion identification is only possible for tracks which project into the fiducial volume of the muon system and the $\mathbf{L A}$ calorimeter and which have momentum greater than $2 \mathrm{GeV} / \mathrm{c}$. Each single charged track is assigned to one of the following seven categories according to the direction and momentum of the track and its interaction with the muon system or LA calorimeters:

1. electron $(e)$,
2. muon $(\mu)$,

3. pion $(\pi)$,

4. not an electron (e)

5. not a muon $(\bar{\mu})$,

6. no particle identification because of track momentum $\left(x_{p}\right)$,

7. no particle identification because of track dircction $\left(x_{d}\right)$.

The details of the criteria for each of the above seven classifications are given in Table 5.1.

Table 5.1. Criteria for classification of a single charged particle.

\begin{tabular}{|c|c|c|c|c|}
\hline & $\begin{array}{c}\text { Momentum } \\
\text { Range }\end{array}$ & $\begin{array}{c}\text { In Muon System } \\
\text { Fiducial Volume }\end{array}$ & $\begin{array}{c}\text { In LA } \\
\text { Fiducial Volume }\end{array}$ & Criteria \\
\hline$c$ & $p>1 \mathrm{GeV} / c$ & & yes & $T E S T 1 \geq 0.85$ \\
\hline$\mu$ & $p>2 \mathrm{GeV} / c$ & yes & & 4 muon layers hit \\
\hline$\pi$ & $p>2 \mathrm{GeV} / \mathrm{c}$ & yes & yes & $T E S T 1<0.85$ \\
& & & yes & $T E S T 1<0.85$ \\
\hline$\vec{c}$ & $1<p \leq 2 \mathrm{GeV} / c$ & & yes & $T E S T 1<0.85$ \\
\hline $\bar{\mu}$ & $p>2 \mathrm{GeV} / c$ & yes & no & $<4$ muon layers hit \\
\hline$x_{p}$ & $p \leq 1 \mathrm{GeV} / c$ & & & \\
& $1<p \leq 2 \mathrm{GeV} / c$ & & no & \\
\hline$x_{d}$ & $p>2 \mathrm{GeV} / c$ & no & no & \\
\hline
\end{tabular}

To separate modes involving one charged hadron, the tagged tau decays are further classified according to the number of neutral energy clusters in the 
hemisphere. For the sample corresponding to the 1 prong tag, photons are defined to be neutral energy clusters with at least $0.65 \mathrm{GeV}$ of energy. The candidates corresponding to the 3 prong tag are less well contained within the LA system than the candidates for the one prong tag. Hence, the probability of detecting a photon in the 3-prong-tag sample is lower. To increase the number of photons detected, the lower energy cutoff is reduced to $0.5 \mathrm{GeV}$ for the 3-prong-tag sample at the expense of increasing the number of spurious plut, tons. These cutoffs are varied to estimate the sensitivity of the measurements to spurious photons.

Candidates in category 3 (a pion), category 4 (not an electron) and category 5 (not a muon) are moved into categories $8,9,10,11$ and 12 if there are one, two, three, four or more than four neutrals in the hemisphere, respectively. Candid:in category 1 (an electron) and category 2 (a muon) with one or more neutrals in the hemisphere are moved into categories 13 and 14 , respectively. These are usually die to hadronic decays in which a hadron posses the electron criteria, or a hadron decays to a muon or punches through the iron absorber simulating a muon. They can also be due to radiative leptonic decays.

To separate modes involving three charged particles, the tagged tau decays with two or three charged particles are separated into two categories according to whether or not these are any neutral energy clusters in the hemisphere.

All of the topologies are shown in Table 5.2 along with the number of tau decays in each, for the tau-candidate samples corresponding to the 1 prong tag and the $\mathbf{3}$ prong tag.
Table 5.2. Topological distribution of the raw tau-candidete samples corresponding to the I prong tag and the 3 prong tag.

\begin{tabular}{|c|c|c|c|c|}
\hline & Particle Identification & \# of photons & 1 prong tag & 3 prong tag \\
\hline 1 & e & 0 & 261 & 219 \\
\hline 2 & $\mu$ & 0 & 170 & 109 \\
\hline 3 & $\pi$ & 0 & 169 & 78 \\
\hline 4 & $\bar{e}$ & 0 & 120 & 189 \\
\hline $\mathbf{5}$ & $\bar{\mu}$ & 0 & 30 & 19 \\
\hline 6 & $\boldsymbol{x}_{\mathbf{p}}$ & & 128 & 166 \\
\hline 7 & $x_{d}$ & & 20 & 92 \\
\hline 8 & $\pi$, e or $\mu$ & 1 & 245 & 182 \\
\hline 9 & $\pi, \bar{\varepsilon}$ or $\tilde{\mu}$ & 2 & 141 & 96 \\
\hline 10 & $\pi, \bar{e}$ or $\bar{\mu}$ & 3 & 42 & 49 \\
\hline 11 & $\pi, \bar{e}$ or $A$ & 4 & 12 & 8 \\
\hline 12 & $\pi, \bar{e}$ or $\bar{\mu}$ & $>4$ & 0 & 1 \\
\hline 13 & e & $>0$ & 27 & 16 \\
\hline 14 & $\mu$ & $>0$ & 7 & 6 \\
\hline 15 & 2 or 3 charged particles & 0 & 120 & 102 \\
\hline 16 & 2 or 3 cirarged particles & $>0$ & 135 & 143 \\
\hline & \multicolumn{2}{|c|}{ Total Number of "jvents } & 1627 & 1475 \\
\hline
\end{tabular}

5.2 THE UNFOLD TECHNIQUE

The likelihood for any set of branching fractions to produce the observed number of decays with each topology is calculated. The best estimate of the branching fractions is that which maximizes the likelihood. This procedure is described in detail in this section. 
Let the number of events observed with topology $i$ be $N_{i}$ and let the total number of observed events be $N=\sum_{i} N_{i}$. Using a Monte Carlo simulation, an efficiency matrix $\epsilon$ is determined whose elements $\epsilon_{i j}$ correspond to the probability of detecting a $\tau$ which decayed via mode $j$ in the ith topology category. Then, for a given set of branching fractions, $B_{j}$, the expected number of events in the $i t h$ topology category, $M_{i}$, is given by

$$
M_{i}=\sum_{j} \epsilon_{i j} B_{j} M
$$

where $M$ is a normalization factor,

$$
M=\frac{N}{\sum_{i} \sum_{j} \epsilon_{i j} B_{j}},
$$

which ensures that $\sum_{i} M_{i}=N$ (i.e. that the total number of expected events equals the total number of observed events). The sum of all the branching fractions is constrained to be equal to one.

The probability of observing $N_{i}$ events for a Poisson distribution with mean $M_{i}$ is

$$
\mathcal{L}_{i}=\frac{M_{i}^{N_{i}} e^{-M_{i}}}{N_{i} !}
$$

The Poisson likelihood function for a particular set of branching fractions $B_{j}$ is then the product of the individual probabilities for each observed topology,

$$
\mathcal{L}=\prod_{i} \mathcal{L}_{i}=\prod_{i} \frac{M_{i}^{N_{i}} e^{-M_{i}}}{N_{i} !}
$$

It is more convenient to maximize the $\ln \mathcal{L}$ since

$$
\ln \mathcal{L}=\sum_{i} N_{i} \ln M_{i}+\text { constants }
$$

To summarize, the procedure is to find the set of branching fractions, $B_{j}$, which results in an 'expected' distribution, $M_{i}$, calculated using the efficiency matrix $\epsilon_{i j}$, which maximizes $\sum_{i} N_{i} \ln M_{i}$.

The minimization program MINUlT ${ }^{41}$ is used to find the set of branching fractions which minimizes the function $f=-\sum_{i} N_{i} \ln M_{i}$. The program also estimates the errors on the branching fractions using the standard technique of associating a change in the $\log$ likelihood with confidence levels for a normal distribution. This assumes that the $\log$ likelihood function is approximately parabolic at the maximum. The relationship between a change in the log likelihood, $\delta f$, and the number-of-standard-deviations confidence level, $n_{\sigma}$, calculated using a normal distribution, is $\delta f=n_{\sigma}^{2} / 2$. So, for example, a one standard deviation confidence level corresponds to a half unit change in $\ln \mathcal{L}$. In practice, once the optimal branching fractions are found, the error on a particular branching fraction is estimated by evaluating the $\ln \mathcal{L}$ for a series of values near the optimal value, remaximizing the log likelihood for the remaining branching fractions at each point.

\subsection{THE EFFICIENCY MATRIX}

Using Monte Carlo simulated events, an efficiency matrix is generated whose elements, $\epsilon_{i j}$, are equal to the efficiency of detecting a $\tau$ which decayed via mode 
$j$ in the ith category described above. Table 5.3 shows the Monte Carlo generated efficiency matrix (actually, the trans, zse of the efficiency matrix $\epsilon_{i j}$ described above) for the 1 prong tag. The first column lists the decay modes and the second column lists the total efficiency for detecting the decay in any of the abovecategories.

The relative efficiencies in this column reflect any biases introduced by the cuts. Any uncestainty in the bias results in an equal fractional uncertainty in the branching fractions.

In the fit, the branching fractions for $\tau^{-} \rightarrow \nu_{\mathrm{r}} \mathrm{K}^{-}$and $\tau^{-} \rightarrow \nu_{\mathrm{r}} \mathrm{K}^{*-}$ are fixed at the best measured values of $(0.6 \pm 0.2) \%$ by $\mathrm{DEL} \mathrm{CO}^{32}$ for $\tau^{-} \rightarrow \nu_{r} K^{-}$and $(1.3 \pm 0.4) \%$ by Mark $I^{2 \theta}$ for $\tau^{-} \rightarrow \nu_{\mathrm{r}} K^{*-}$.

The efficiency matrix for the 3 prong tag is shown in Table 5.4. Note that this matrix is less 'diagonal' than that for the 1 prong tag. For example, if a tau decays via the mode $\tau^{-} \rightarrow \nu_{\tau} \mu^{-} \nu_{\mu}$ and makes it into the tau candidate sample, the probability that it is classified as a muon (category 2) is about $58 \%$ for the 1 prong tag and about $34 \%$ for the three prong tag. This difference is duc to the different fiducial volume criteria for the two lags. Recall that for the 1 prong tag, the single charged particle in the hemisphere must pass through the active volume of both the LA calorimeter and the muon system. Since the decay products of the two tau's in the pair are quite collinear, tau decay eandidates opposite the 1 prong tag are likely to also pass through the active parts of the detector. The geometrical eriteria for the 3 prong tag are much looser.

These efficiency matrices are only the first order approximation to the actual 


\begin{tabular}{|c|c|c|}
\hline 2 & & 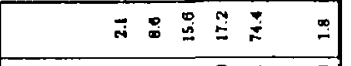 \\
\hline & 5 & $\stackrel{i}{i} \stackrel{R}{i}$ \\
\hline & & 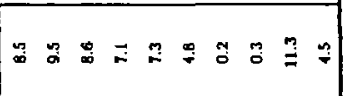 \\
\hline & & $\check{2}$ \\
\hline & $=$ & 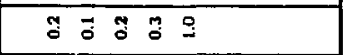 \\
\hline & $\wedge$ & 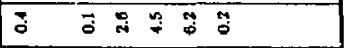 \\
\hline & & : \\
\hline & & $\ddot{g}$ \\
\hline & 75 & 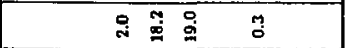 \\
\hline & & 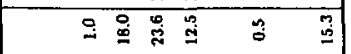 \\
\hline & $=$ & $\square$ : \\
\hline & 12 & స \\
\hline & 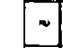 & 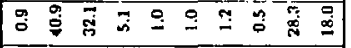 \\
\hline & 5 & 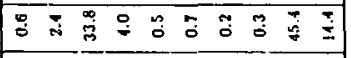 \\
\hline & 3 & 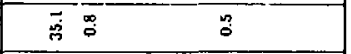 \\
\hline & 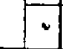 & 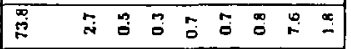 \\
\hline & $\frac{\sqrt{3}}{3}$ & 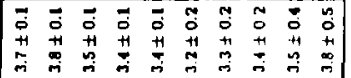 \\
\hline & & 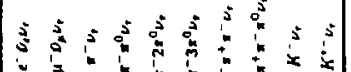 \\
\hline
\end{tabular}

efficiency matrices used in the analysis since they are based totally on the Monte Cario. Its accuracy depends on how well the Monte Carlo simulates the momentum distribution, the angular distribution and the particle identification efficiencies for the decay products. In the next section, the Monte Carlo is described. In the following sections, the efficiency checks using real data are discussed.

\subsection{Monte Carlo simulation}

The Monte Carlo simulation is based on an event generator for $e^{+} e^{-} \rightarrow$ $\tau^{+} \tau^{-}(\gamma)$ written by F. A. Berends and R. Kleiss ${ }^{42}$ which includes the emission of real photons from the initial and final state leptons, virtual radiative corrections to the lepton-photon vertex, multiple photon exchange and vacuum polarization corrections. Although the tau leptons produced in $e^{+} e^{-}$annihilation at PEP are not polarized, the spin of one member of the pair is correlated with the spin of the other. ${ }^{16}$ These correlations are included in the Monte Carlo.

The generated $r$ 's decay approximately according to the measurcd branching fractions. The exact branching fractions used in the Monte Carlo are irrelevant for this analysis The following decay modes are included:

$$
\begin{aligned}
& \tau^{-} \rightarrow \nu_{\tau} e^{-} \bar{\nu}_{e}, \\
& r^{-} \rightarrow \nu_{\tau} \mu^{-} \bar{\nu}_{\mu}, \\
& \tau^{-} \rightarrow \nu_{\tau} \pi^{-}, \\
& \tau^{-} \rightarrow \nu_{r} \rho^{-}, \\
& \tau^{-} \rightarrow \nu_{\tau} A_{1}^{-}, \\
& \tau^{-\cdots} \rightarrow \nu_{r}(4 \pi)^{-},
\end{aligned}
$$




$$
T^{-} \rightarrow \nu_{\mathrm{r}} K^{-} \text {, }
$$$$
\text { and } r^{-} \rightarrow \nu_{\tau} K^{*-} \text {. }
$$

The correct angular distribution with respect to the $\tau$ spin is incorporated in the Monte Carlo for the e, $\mu, \pi, \rho, K$ and $K^{*}$ decay modes. Also, the angular distribution of the charged pion from the decay of the a reflects the helicity of the $\rho$ correctly. The angular distribution for the $A_{1}$ and the $4 \pi$ mode is isotropic in the $\tau$ rest framc. This is not strictly correct but the effect is small because of the large number of particles in the final state. A Breit-Wigner mass shape with the known mass and width is used for the $\rho, K^{*}$ and $A_{1}$. The mass of the $4 \pi$ mode is produced according to isotropic phase space.

All particles are propagated through the detector, decaying according to the known lifetimes and branching fractions. Multiple scattering, bremsstrahlung and photon conversions are simulated. The simulation of electromagnetic interactions in the LA system is based on the EGS shower code. ${ }^{43}$ The simulation of hadronic interactions in the LA system is hased on a library of real pion interactions recorded in a $4 \mathrm{GeV} / c$ pion beam test. The energy deposited in four strips on either side of the track trajectory in each layer is scaled by a factor which depends on the total shower energy and the track momentum, and which maintains the minimum ionizing peak. The muon system simulation includes the effects of multiple scattering, range straggling, proportional tube inefficiencies, tracking extrapolation errors, hadron punchthrow $-h$, and decay of hadrons to muons in fight.

The charged particle momentum resolution of the drift chamber, the muon system inefficiencies, and the energy resolution of the LA calorimeter are adjusted in the Monte Carlo to simulate the real data. However, discrepancies still remain and these are discussed in detail in the next section.

\subsection{Particle Identification EfFiciency}

To determine the true particle identification efficiencies, tracks corresponding to particles with known identification in the data are used. The efficiency for lepton identification is estimated using two sources of known leptons in the data: single photon production of $e^{+} e^{-}$and $\mu^{+} \mu^{-}$pairs, and two photon production of $e^{+} e^{-}$ and $\mu^{+} \mu^{-}$pairs. Together these samples cover most of the relevant momentum range. The selection criteria for these events sre described in Appendix A. The three tracks in the 3 prong tag are used to determine pion identification efficiencies.

\subsubsection{Muon Identifiration}

The $\mu / \pi$ discrimination algorithm is described in Chapter 3 . Recall that $\mu / \pi$ discrimination is attempted only if the track is expected to leave hits in all four layers if it is a muon; i.e., the track trajectory projects through all four layers and the particle has sufficient momentum to reach the fourth layer if it is a muon. The probability that a muon has associated hits in all four layers of the muon system is shown in Figure 5.1 as a function of momentum for muons in the data and primary muons from $r$ decay in the Monte Cario. The muon identification efficiency for the muon candidates in the data has been corrected for a small contamination from taupair events. The muon identification efficiency is systematically about $3 \%$ higher in 
the Monte Carlo than in the data. The appropriate elements corresponding to the muon decay mode in the efficiency matrix are corrected for this fact.

The identification efficiency is also studied as a function of the polar angle of the irack. Since low momentum tracks scatter through larger angles than high momentum tracks when passing through material, a muon with low momentum near the edge of the module is more likely to scatter out of the modulc and fail the criteria for a muon. The efficiency is lower near the edge of the module for low momentum tracks while there is little dependence of the efficiency on the polat angle for high momentum tracks. This dependence on poler angle is seen in both the Monte Carlo and the data, with the identification efficiency in the Monte Carlo being consistently higher. Of course, the efficiency also depends slightly on the azimuthal angle for the same reason.

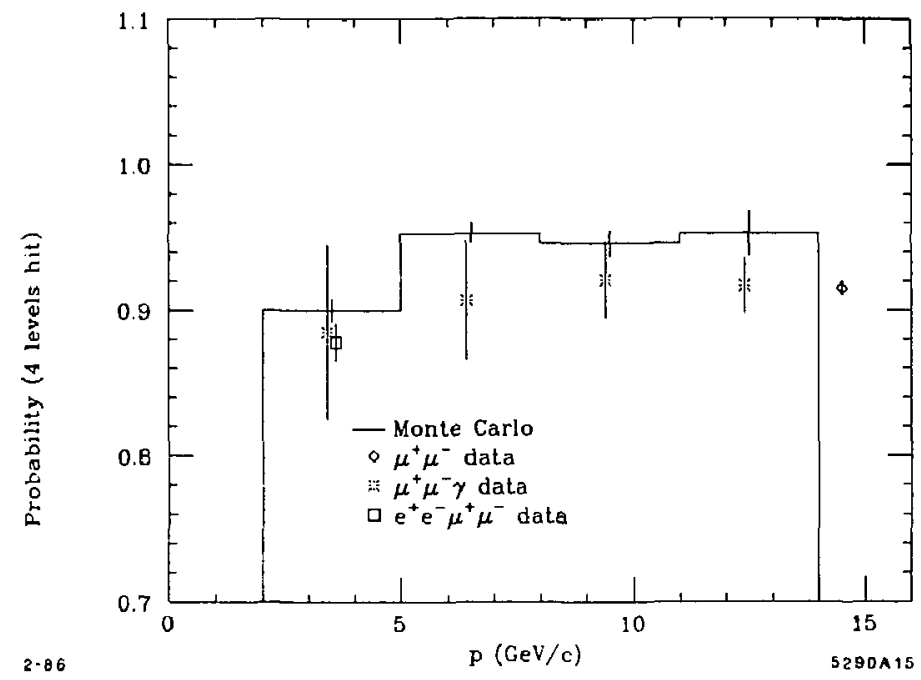

Figure 5.1. Muon identification efficiency for muons which are expected to penetrate to the fourth layer of the muon system. The histogram corresponds to primary muons from $r$ decay in the Monte Carlo. 
5.5.2 Electron Identification

The $e / \pi$ discrimination algorithm is described in Chapter 3. Recall that $e / \pi$ discrimination is attempted only if the particle momentum is greater than $1 \mathrm{GeV} / \mathrm{c}$ and the track trajectory projects into the fiducial volume of the LA calosimeter. The probability that TEST1 $>0.85$ for an electron is shown in Figure 5.2 as a function of momentum for electrons in the data and primary electrons from $\tau$ decay in the Monte Carlo. For the sample of known electrons from radiative Bhabha events, thr electron candidate is only used if the photon is in a different LA module. The electron identification efficiency is consistently about $3 \%$ higher in the Monte Carlo than in the data. The appropriate elements corresponding to the electron decay mode in the efficiency matrix are corrected for this fact. No significant dependence of the efficiency on polar angle is seen in the data or in the Monte Carlo.

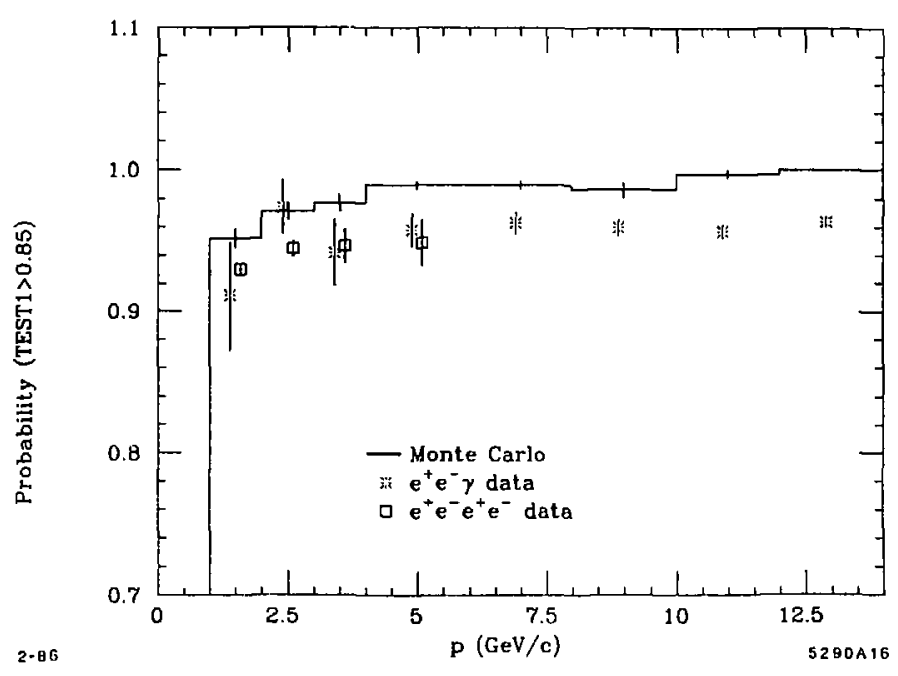

Figure 5.2. Electron identification efficiency for electrons which project in to the fiducial volume of the LA calorimeter. The histogram corresponds to primary electrons from $r$ decay in the Monte Carlo. 
5.5.3 Pion Identification

A pion is defined to be a track which meets the momentum and fiducial volume criteria for $\mu / \pi$ and $e / \pi$ discrimination and does not satisfy the requirements to be identified as a muon or an electron; i.e., the track does not have associated hits in all four iayers of the muon system and TEST1 is less than 0.85 .

The probability that a pion which meets the momentum and fiducial volume criteria for muon identification has associated hits in all fous levels of the muon system is determined using pions from three prong decays of the tau. The probability is $(1.4 \pm 0.4) \%$ in the data and $(0.6 \pm 0.2) \%$ in the Monte Carlo. It is esimated that about half of the pions in the data which pass the criteria for a muon are due to the decay of the $\pi$ to $\mu \nu_{\mu}$ in flight, and about half are due to hadronic punchthrough and track overlap. The discrepancy between the data and the Monte Carlo is probably due to the following two inadequacies in the Monte Carlo. The Monte Carlo does not simulate secondaries from pion interactions correctly and does not include random noise in the muon system. The appropriate elements in the efficiency matrix are corrected for this discrepancy by fixing the probability of a hadron satisfying the muon criteria at $1.4 \%$ if it satisfies the momentum and fiducial volume criteria for muon identification. The probability of misidentifying a single prong hadron as a muon is actually slightly lower than this because there is no overlap of hits from other tracks. However, the difference has a negligible effect on the meak' rred branching fractions.

The probability that a pion which meets the momentum and fiducial volume criteria for electron identification and has TEST1 $<0.85$ is shown in Figure 5.3 as a function of momentum for pions in the 3 prong tag, in the data and in the Monte Carlo. For low momentum, the identification efficiency for pions is about $3 \%$ lower in the Monte Carlo than in the data. The efficiency for $e / \pi$ discrimination also depends on the number of photons and other charged tracks in the vicinity of the track. For example, the pion identification efficiency is about $1.3 \%$ higher for pions which are from a three prong decay of the tau with no detected neutrals than for those with a detected neutral, in both the data and the Monte Carlo.

For most decay modes with one charged hadron, the decay is classified as an electron accompanied by at least one neutral if the charged hadron is misidentified as an electron. This does not mix with the electron decay mode unless radiative decays are important. 


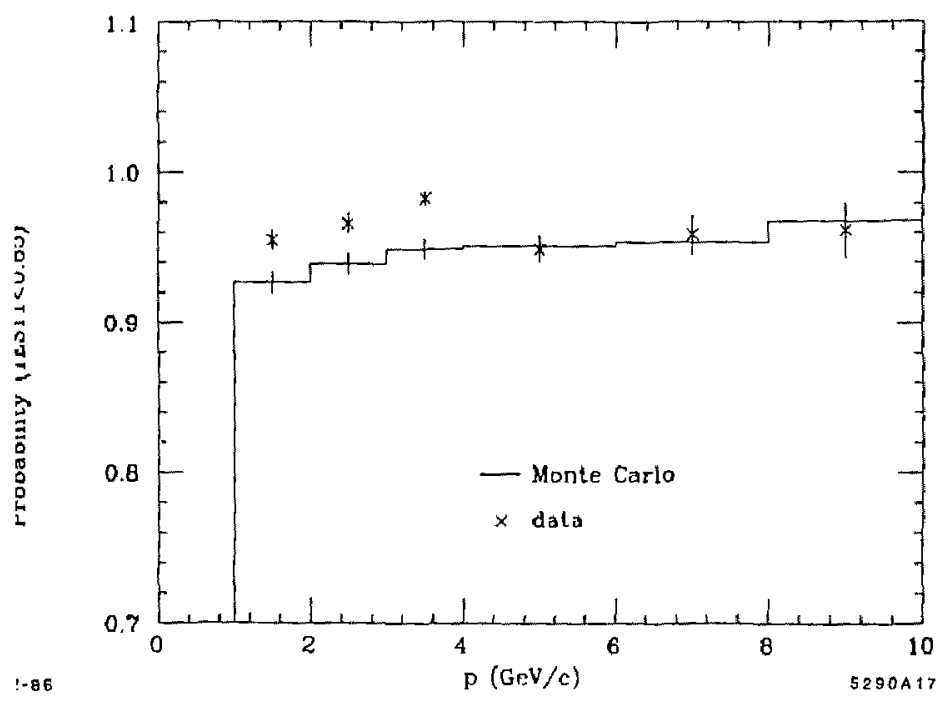

Figure 5.3. e/ $\pi$ discrimination efficiency for pions. The histogram corresponds to pions in the Monte Carlo. The symbol $x$ corresponds to pions in the data.

\subsection{ANGULAR Distribution}

The angular distribution of the $r$ candidates in the sample affects the particle identification efficiencies because of the limited active volume of each detector component. The decay products from the two leptons are quite collinear because of the large thrust due to the high n:omentum of the $r$ in the laboratory frame. Therefore, the angular distribution of the decay products in the $r$ sample is strongly dependent on the angular distribution of the decay products in the tag.

The angular distribution of the single charged track in the I prong tag is shown in Figure 5.4 for the data and the $r$ Monte Carlo. The top tigure shows the $|\cos \theta|$ distribution where $\theta$ is the polar angle; the bottom distribution shows the azimuthal angle $\phi$ between the track and the horizontal. Recall that for lepton rejection, the charged track in the 1 prong tag must project into the active volume of the muon system and the LA calorimeter. The large gaps in $\phi, 45^{\circ}$ from the horizontal, correspond to gaps between the four walls of the muon system. The smaller gaps in $\phi_{1}$ at $0^{\circ}$ and $90^{\circ}$ fiom the horizontal, correspond to the gaps between the eight $\mathrm{LA}$ modules. The agreement between the Monte Cario and the data is fairly good. A slightly smaller fraction of the charged tracks in the 1 prong tag are near the edges of the detector elements in $\theta$ in the data than in the Monte Carlo.

The distribution of the polar angle for the three charged tracks in the 3 prong tag is shown in Figure 5.5 for the data and the $r$ Monte Carlo. The agreement is quite good. Since there is no electron or muon identification criteria applied to the 3 prong tag, the distribution of tracks is flat in $\phi$. 

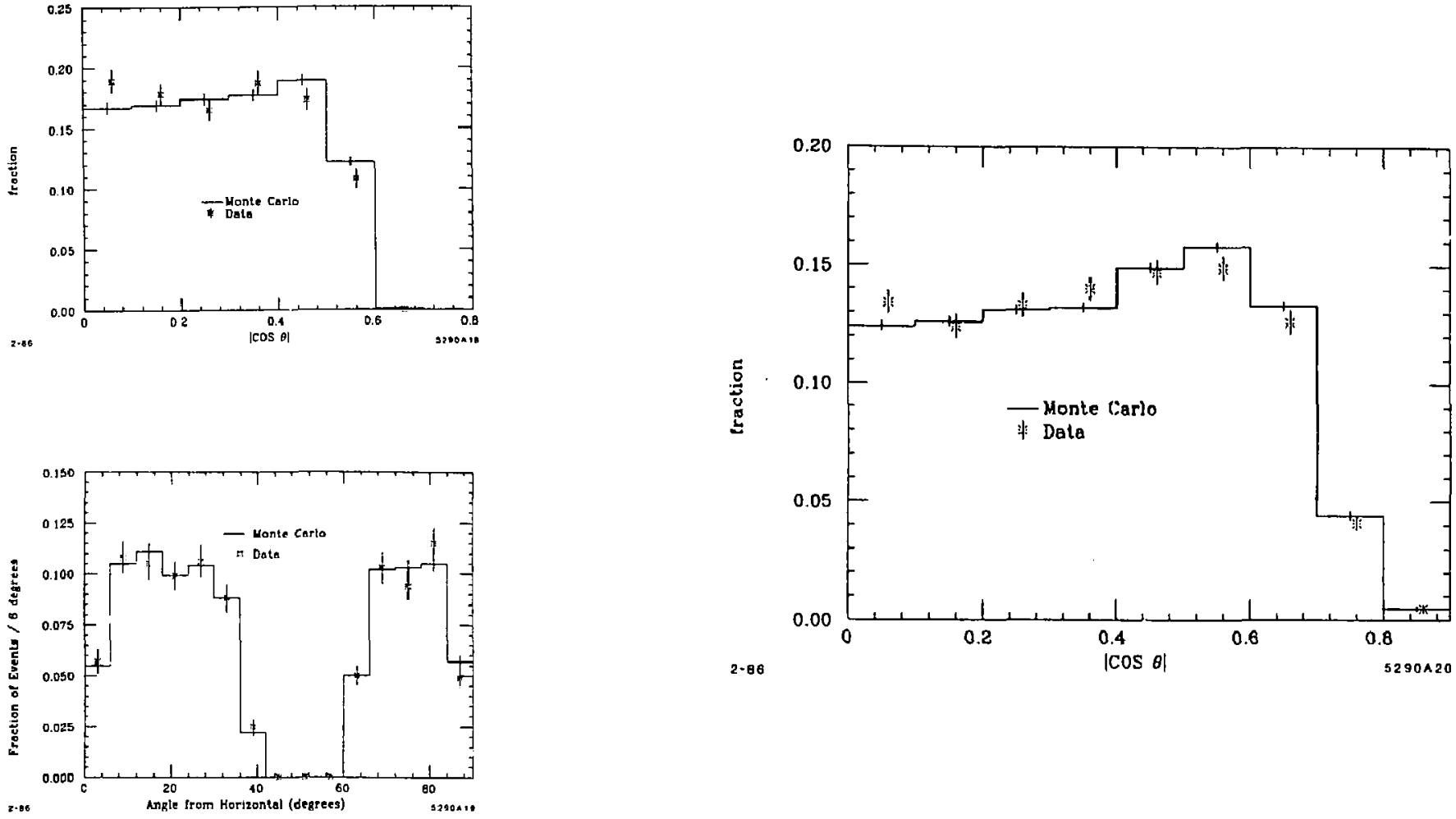

Figure 5.4. Angular distribution of the single charged track in the 1 prong tag. The histogram corresponds to the Monte Carlo and the symbol $\times$ corresponds to the data. The top figure shows the distribution of tracks as a function of $|\cos \theta|$ where $\theta$ is the polar angle. The bottom figure shows the distribution of tracks as a function of the azimuthal angle relative to the horizontal.

Figure 5.5. Angular distribution of the three charged tracks in the 3 prong tag. The histogram corresponds to the Monte Carlo and the symbol $x$ corresponds to the data. 
5.7 MOMENTUM Distribuı.

The momentum distribution of the tagged particles affects the particle identification efficiency because of the minimum momentum cut-off of $1 \mathrm{GeV} / \mathrm{c}$ for electron identification and $2 \mathrm{GeV} / \mathrm{c}$ for muon identification. The momentum distribution of all tagged one prongs in the tau-candidate sample is shown in Figure 5.6 and Figuse 5.7 for the 1 prong tag and the 3 prong tag, respectively. The distribution is shown for both the data and the Monte Carlo. Or course, the branching fractions affect this distribution since the momentum distribution is different for each decay mode. The branching fractions used in the Monte Carlo are close to those measured with the data. The fact that the agreement is quite good indicates that the background contributions to the samples are not large. The backgrounds are estimated in the following chapter.

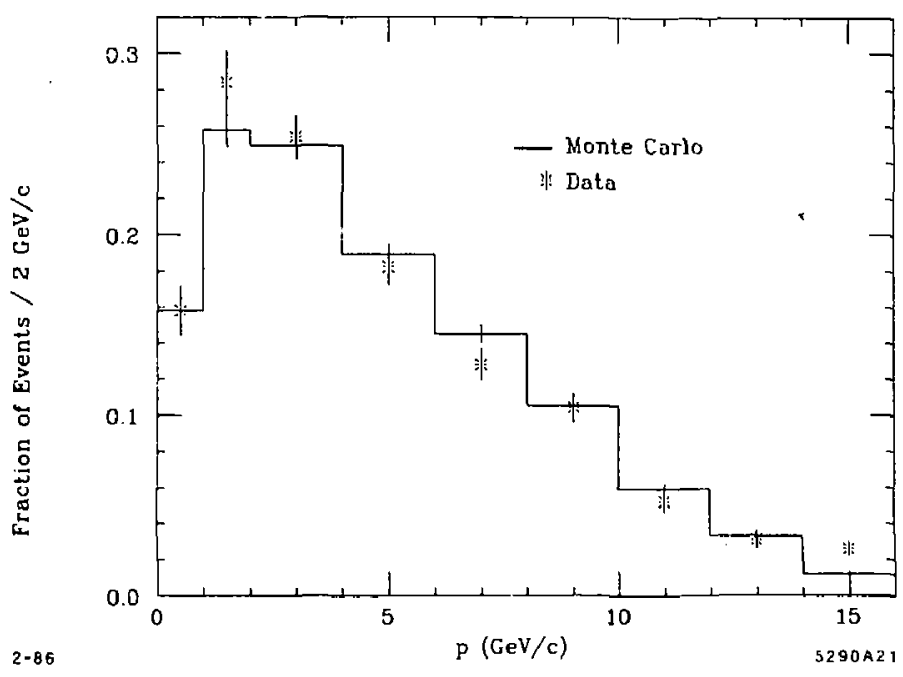

Figure 5.6. Momentum distribution of one prongs in the tau-candidate sample corresponding to the 1 prong tag. The histogram corresponds to the Monte Carlo and the symbol $x$ corresponds to the data. 


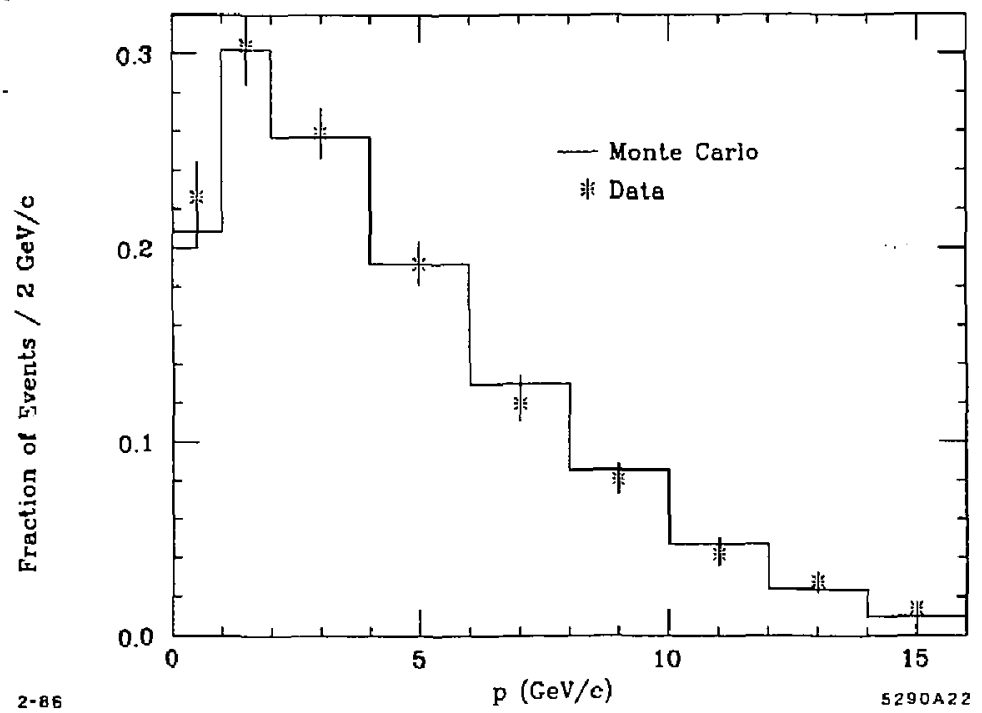

Figure 5.7. Momentum distribution of one prongs in the tau-candidate sample corresponding to the 3 prong tag. The histogram corresponds to the Monte Carlo and the symbol $x$ corresponds to the data.

\section{Backgrounds}

The contribution to the samples from sources other than tau-pair production through single photon annihilation of the $e^{+} e^{-}$pair is discussed and estimated in this chapter. The potential sources of background are single photon production of $e^{+} e^{-}$and $\mu^{+} \mu^{-}$pairs, two floton production of $e^{+} e^{-}, \mu^{+} \mu^{-}, \tau^{+} \tau^{-}$äfid $q \bar{q}$ pairs, and single photon production of $q$ pairs leading to hadronic jets. The background from two photon production of tau pairs and $q \bar{q}$ pairs is discussed for both samples at once. Then the other sources of background are discussed for the two tags separately since they contribute to the two tags in quite different ways.

\subsection{Background from Two Photon Production of Tau Pairs}

A sample of Monte Carlo simulated events of two photon production of tau pairs, equivalent to an integrated luminosity of about $270 \mathrm{pb}^{-1}$, is subjected to all of the selection criteria for each tag. The number of charged particles which pass the cuts is used to predict the total background in the tau-candidate sample. The background contribution from $e^{+} e^{-} \rightarrow e^{+} e^{-} \tau^{+} \tau_{+}^{-}$, expected in $207 p b^{-1}$, is shown in Table 6.1 for the 1-prong-tag and 3-prong-tag tandidate samples. This background constitutes $(2.1 \pm 0.3) \%$ and $(1.0 \pm 0.2) \%$ of the signal for the samples corresponding to the 1 prong tag and the 3 prong tag, respectively.

About a third of the candidates in the 1-prong-tag sample are classified as electrons. The remainder are scattered among the other categories. The fraction identified as electrons is significantly larger than the frnction identified as muons or pions for several reasons. First of all, recall that an event with total energy 
pass than an event with a muon in it. Secondly, the momentum spectrum of the

Table 6.1. Background from $e^{+} e^{-} \rightarrow e^{+} e^{-} \tau^{+} \tau^{-}$in $207 \mathrm{pb}^{-1}$ for the taucandidate samples corresponding to the 1 prong tag and the 3 prong tag.

\begin{tabular}{|c|c|c|c|c|}
\hline & Particle Identification & \# of photons & 1 prong tag & 3 prong tag \\
\hline 1 & $\varepsilon$ & 0 & 11 & 3.1 \\
\hline 2 & $\mu$ & 0 & 1.5 & 0 \\
\hline 3 & $\pi$ & 0 & 0.8 & 0.8 \\
\hline 4 & e & 0 & 3.1 & 1.5 \\
\hline 5 & $a$ & 0 & 0.8 & 0 \\
\hline 6 & $x_{p}$ & & 0.8 & 1.5 \\
\hline 7 & $x_{d}$ & & 0.8 & 3.1 \\
\hline 8 & $\pi, \bar{e}$ or $\bar{\mu}$ & 1 & 5.4 & 3.1 \\
\hline 9 & $\pi, \bar{c}$ or $\bar{\mu}$ & 2 & 3.1 & 0.8 \\
\hline 10 & $\pi, z$ or $\mu$ & 3 & 0 & 0 \\
\hline 11 & $\pi, \bar{e}$ or $\bar{\mu}$ & 4 & 0.8 & 0 \\
\hline 12 & $\pi, \bar{c}$ or $\bar{\mu}$ & $>4$ & 0 & 0 \\
\hline 13 & $e$ & $>0$ & 0 & 0 \\
\hline 14 & $\mu$ & $>0$ & 0 & $\mathbf{0}$ \\
\hline 15 & 2 or 3 charged particles & 0 & 3.1 & 0.8 \\
\hline 16 & 2 or 3 charged particles & $>0$ & 3.1 & 0 \\
\hline & \multicolumn{2}{|c|}{ Total Number of Events } & $35 \pm 5$ & $15 \pm 3$ \\
\hline
\end{tabular}

less than $E_{\text {beam }} / 2$ is not written on the data tapes unless it contains an electron with $p>1.25 \mathrm{GeV} / c$ (where an electron is defined using information from the TOF system or the LA calorimeter), or a track with sssociated hits in three of the four muon chamber levels. Since the LA calorimeters and TOF system cover a larger solid angle than the muon system, an event with an electron in it is more likely to charged particles is peaked at the low end, and the minimum momentum required for muon or pion identification is $2 \mathrm{GeV} / \mathrm{c}$, while that for electron identification is only $1 \mathrm{GeV} / \mathrm{t}$. This background constitutes $(4.4 \pm 1.2) \%$ of the electron signal in the sample corresponding to the 1 prong tag.

\subsection{BAGKGROUND FROM TWO PHOTON PRODUCTION OF $q-\bar{q}$}

Recall that the overall criteria which an event must meet include $E_{c h}>$ $E_{\text {beam }} / 4$ and $E_{t o t}>E_{\text {beam }} / 2$ if none of the tracks in the event is identified as an electron or a muon (see section 4.2). This eliminates most background from two photon production of $q 9$ pairs. In addition, the cut on $p^{*}$ for the 3 prong tag, and the requirement of a track with at least $2 \mathrm{GeV} / \mathrm{c}$ momentum at large $|\cos \theta|$ for the 1 prong tag, provide rejection against this background.

\subsection{Backgrounds to the I Prong TAg}

6.3.1 Leptonic Backgrounds

The backgtound due to leptons passing the lepton rejection criteria for the single charged partcle in the 1 prong tag is estimated as follows. All of the criteria for the 1 prong tag, except the lepton rejection cuts, are applied to Monte Carlo simulations of single and two photon production $u i$ lepton pairs. This results in a significantly large number of events passing the momentum cuts and fiducial volume requirements of the 1 prong tag. Then the efficiency for lepton rejection, determined

* In this section, the term lepton refers to electrons and muons only. 
from PEP data, is used to estimate the background from these sources. This methad has several advantages. It does not depend on the Monte Curlo simulation of the lepton interactions with thi $i \Lambda$ calorimeter and the muon gystem, and it results in an estimate of the background with small statistical etrors without generating an enormous number of Monte Carlo events.

The muon and electron rejection criteria are described in Chapter 3 . The efficiency for lepton rejection is estimated using the two sources of known leptons in the data described in Appendix A: single photon and two photon production of $e^{+} e^{-}$and $\mu^{+} \mu^{-}$pairs.

\subsubsection{Muon Rejection Efficiency}

Of the 4923 muons in the sample of muon candidates from the data passing the fiducial volume criteria for muon rejection (at least three sigma from the edges of the muon system), 17 pass the rejeetion criteria (hits in none of the four layers, or in the first and/or last layer only). Plots of these events were handscanned. Of the 17 muon candidates, 5 are obviously electrons. Since these $\mu-e$ events are probably from T-pair production, they are eliminated from the sample. Of the remaining 12 muon candidates, 5 look like pions, probably also due to r-puir production.* These muon candidates are also eliminated from the sample leaving 7 muons which

- The number of electrons and pions from tau decay expected to contaminate the muon-candidate sample is also estimated using Monte Carlo generated r-pair events. The expected number of $\mu-\epsilon$ and $\mu-\pi$ events agrees well, within otatistical errors, with the number found through hand-scanning. actually pass the muon rejection critcria. Therefore, the moun rejection inefficiency is $(0.14 \pm 0.05) \%$.

\subsubsection{Electron Rejection Efficiency}

Of the 15555 electron candidates in the data with momentum greater than $1 \mathrm{GeV} / \mathrm{c}$ and less than $14 \mathrm{GeV} / \mathrm{c}$, projecting into the active volume of the $\mathrm{LA}$ calorimeter, 62 pass the rejection criteria $(E / p<0.5)$. Plots of these events were handscanned. Of the 62 electron candidates, 11 are obviously muons. Again, since these $e-\mu$ events are probably from $\tau$ pair production, they are eliminated from the sample. Of the remaining 51 candidates, 16 deposit about $300 \mathrm{MeV}$ of energy in the LA calorimeter, which is the amount a minimum ionizing particle is expected to deposit. These are probably also due to $\tau$-pair production and are eliminated from the sample. Of the remaining 35 candidates, 12 events correspond to $e^{+} e^{-}$ production in which the electron candidate is mistracked (usually in the $\boldsymbol{\varepsilon}$ direction) resulting in the energy in the $\mathbf{L A}$ calorimeter being identified as a photon. In each of these cases, the momentum of the charged track as measured by the drift chamber plus the energy of the nearby neutral energy cluater is well above the maximum total energy of $12 \mathrm{GeV}$ for the 1 prong tag. These electron candidates are also

- The number of muons and pions from tau decay expected in the electron candidate sample is also estimated using Monte Carlo generated r-pair events. The expected number of $e-\mu$ and $e-\pi$ events agrees well, within statistical errors, with the number found through hand-scanning. 
eliminated from the asmple leaving 21 electrons which actually pass the $E / p$ cut.

Therefore, the electron rejection inefficiency is $(0.15 \pm 0.03) \%$

6.3.4 Background from Single Photon Production of Lepton Pairs

The Monte Carlo for single photon production of lepton pairs is based on the generator of Behrends and Kleiss ${ }^{42}$ which includes $O\left(\alpha^{3}\right)$ effects. In the $e^{+} e^{-}(\gamma)$ generator, at least one of the charged leptons is emitted with an angle of at least $40^{\circ}$ to the beam line. In both the $e^{+} e^{-}(\gamma)$ and $\mu^{+} \mu^{-}(\gamma)$ generator, the maximum photon energy is $90 \%$ of the beam energy. A sample of Monte Carlo simulated events is subjected to all of the aelection criteria for the 1 prong tag except the lepton rejection cut. The number of charged particles which pass these criteria is shown in Table 6.2. Most of the everts which pass the selection criteria correspond to the produrtion of a photon and two leptons with aproximately equal energy in which the photon travels down the beam pipe and is undetected. Using the electron and muon rejection inefficiencies determined from known electrons and muons in the data leads to the estimated backgrounds shown in the table. The distribution of the background among the various topologies is also determined from the Monte Carlo and the major contributions are shown in Table 6.4.

\subsubsection{Background from Two Photon Production of Lepton Pairs}

Two photon production of lepton pairs with a minimum invariant mass of $3 \mathrm{GeV} / \mathrm{c}^{2}$ is simulated using an equivalent-photon-approximation generator. ${ }^{14} \mathrm{~A}$ sample of Monte Carlo simulated events equivalent to an integrated luminosity of
Table 6.2. Background from single photon production of lepton pairs.

\begin{tabular}{|c|c|c|}
\hline Event Type & $e^{+} e^{-} \rightarrow e^{+} e^{-}(\gamma)$ & $e^{+} e^{-} \rightarrow \mu^{+} \mu^{-}(\gamma)$ \\
\hline \# of Monte Carlo simulated events & 80000 & 40000 \\
\hline Equivalent integrated luminosity & $30.8 p^{-1}$ & $317 p b^{-1}$ \\
\hline $\begin{array}{c}\text { \# of tracks which survive all cuts for } \\
1 \text { prong tag except lepton sejection cuts }\end{array}$ & 446 & 555 \\
\hline Lepton rejection inefficiency & $(0.15 \pm 0.03) \%$ & $(0.14 \pm 0.05) \%$ \\
\hline \# of background events in $207 p b^{-1}$ & $4.5 \pm 0.9$ & $0.5 \pm 0.2$ \\
\hline
\end{tabular}

about $14 \mathrm{pb}^{-1}$ is subjected to all of the selection criteria for the 1 prong tag exeept the lepton rejection cut. The number of charged particles which pass these criteria is shown in Table 6.3. Using the electron and muon rejection inefficiencies determined from known electrons and muons in the data leads to the estimated backgrounds shown in the table. The distribution of the background among the various topologies is also determined from the Monte Carlo and the major contributions are shown in Table 6.4.

Table 6.3. Background from two photon production of lepton pairs.

\begin{tabular}{|c|c|c|}
\hline Event Type & $e^{+} e^{-} \rightarrow e^{+} e^{-} e^{+} e^{-}$ & $e^{+} e^{-} \rightarrow e^{+} e^{-} \mu^{+} \mu^{-}$ \\
\hline \# of Monte Carlo simulated events & 11093 & 10927 \\
\hline Equivalent integrated luminosity & $13.2 \mathrm{pb}$ & $14.9 \mathrm{pb}^{-1}$ \\
\hline $\begin{array}{c}\text { \# of tracks which survive all cuts for } \\
1 \text { prong tag except lepton rejection cuts }\end{array}$ & 199 & 228 \\
\hline Lepton rejection inefficiency & $(0.15 \pm 0.03) \%$ & $(0.14 \pm 0.05) \%$ \\
\hline \# of background events in $207 \mathrm{pb}^{-1}$ & $4.7 \pm 0.9$ & $4.4 \pm 1.6$ \\
\hline
\end{tabular}


6.3.6 Background from Single Photon Production of $q-q$

The background in the tau-candidate sample from hadronic events is estimated from the invariant mass distribution of the candidate tau decays. The background is calculated for the sample of candidates with one charged particle and higher charged multiplicities, separately.

The procedure is outlined here for the candidates with more than one charged particle. The invariant mass of the charged particles and neutrals is calculated for each candidate tau decay. In the sample selected from the data, $(14 \pm 2) \%$ of the candidates have an invariant mase greater than $2.5 \mathrm{GeV} / \mathrm{c}^{2}$. For a similar sample of tau decays in the Monte Carlo, only $(2.8 \pm 0.7) \%$ have mass greater than $2.5 \mathrm{GeV} / \mathrm{c}^{2}$. Also, a sample of hadronic eventg with three charged particles in one hemisphere and at least six charged particles in the other hemisphere is selected from the data and used to determine that $(55 \pm 2) \%$ of hadronic three prong jets have mass greater than $2.5 \mathrm{GeV} / \mathrm{c}^{2}$. These numbers are used to estimate that $28 \pm 6$ of the 255 two and three prong tau candidates with mass less than $2.5 \mathrm{Gel} / \mathrm{c}^{2}$ are due to hadronic background. In the sample of hadronic three prongs (with mass less than $\left.2.5 \mathrm{GeV} / \mathrm{c}^{2}\right)$ from the data, $(74 \pm 2) \%$ have at least one neutral in the threc prong jet. This is used to determine the distribution of the background between the topologies with zero or greater than zero neutrals.

A similar analysis applied to the candidates with one charged particle leads to an estimate of $24 \pm 10$ hadronic background events among the 1372 candidates. The hadronic sample with one charged particle in one hemisphere and at least six in the other hemisphere is used to determine the distribution of the background evente among the topologies with various neutral multiplicities.

\subsection{B.igkground TO THE 3 PRONg TAG}

6.4.1 Background from Single or Two Photon Production of Lepton Pairs

A potential source of background to the 3 prong tag is single or two photon production of iepton pairs with intial or final state radiation in which the radiated photon converts to an $e^{+} e^{-}$pair. The dominant potential source is radiative Bhabha events because of the large cross section for this process. The criterja which eliminate this background are the rejection of events in which

- an oppositely charged pair of tracks in the 3 prong tag has a emall opening angle (this pair-finding algorithm is described in Sec. 3.4), or

- the total energy (charged plus neutral) in the 3 prong tag is greater than $14 \mathrm{GeV}$.

The pair-finding algorithm is very efficient for radiative Bhabha events for the following reasons. First, since the event has three tracks in one hemisphere, asymmetric conversions in which one of the charged particles from the pair is not detected will not contribute to the background. Also, since the photon is typically emitted at a very small angle to the electron, the opening angle between the members of the pair and the primary electron will also be small. Therefore, even if one member of the pair is not well tracked, the other member of the pair and the primary electron are likely to satisfy the criteria for a pair if they are oppositely charged. Finally, because of the low multiplieity of the event, the tracking quality 
is quite good and hence the pair-finding efficiency is high.

From Monte Carlo generated events, the estimated background due to lepton pairs with a conversion is negligible. A handscan of all events in the data with the tau candidate opposite the 3 prong tag identified as an electron, muon or pion reveals four, zero and one ovent from these sources for the electron, muon and pion topologies, respectively.

\subsubsection{Background from Single Photon Production of $q-\bar{q}$}

A Monte Carlo simulation is not used to simulate the background from this process because the low end of the multiplicity distribution depends heavily on the fragmentation process used in the Monte Carlo. A sample of hadronic events with three charged particles in one hemisphere and at least six charged particles in the other hemisphere is selected from the date using all of the selection criteria for the 3 prong tag except the cute on $p^{*}$ and the invariant mass of the 3 prong side. From this sample, it is determined that $(14.2 \pm 1.6) \%$ of 3 prong hadronic jets pass the $p^{*}$ cut $\left(p^{*} \leq 750 \mathrm{MeV} / \mathrm{c}\right)$, and $(52 \pm 6) \%$ pass the mass cut $\left(m_{3}<1.7 \mathrm{GeV} / \mathrm{c}^{2}\right)$ after the $p^{*}$ cut has been applied. From a Monte Carlo simulation of $r$-pair production, it is determined that $(84 \pm 1) \%$ of tau decays to three charged particles pass the $p^{*}$ cut.

The sample of $\mathrm{r}$ candidates tagged with the 3 prong tag contains 567 decays with two or three charged particies before the $p^{*}$ and invariant mass cuts have been applied to the 3 prong tag, and 265 events after the $p^{*}$ cut. Using the known fraction of hadronic jets and tau decays which survive the $p^{\star}$ cut, one can calculate that the sample of 265 decays contains $43 \pm 6$ hadronic jets. Since the invariant mass cut on the 3 prong tag removes a further $(52 \pm 6) \%$ of the hadronic events, the hadronic background in the two and three prong sample, after the $p^{*}$ and mass cuts on the 3 prong tag, is $22 \pm 4$ events. After this, a cut of $2.5 \mathrm{GeV} / \mathrm{c}^{2}$ is made on the invariant mass of the tau candidates opposite the three prong tag. This also eliminates some of the hadronic background, leaving a background of $10 \pm 3$ events or $(4 \pm 2) \%$ of the total two and three prong sample.

A similar analysis applied to the sample of one prong $\tau$ candidates tagged with the 3 prong tag leads to an estimated hadronic background of $10 \pm 2$ events or $(0.8 \pm 0.2) \%$ of the one prong sample.

The distribution of the hadronic background among topologies with different numbers of neutrals is determined from the sample of events with a one or three prong jet versus a six prong jet in the data. These backgrounds are summarized in Table 6.5.

6.5 SUMMARY OF BACKGROUNDS

The barkground estimates for the tau candidates opposite a 1 prong tag and a 3 prong tag are summarized in Table 6.4 and Table 6.5, respectively. The topological categories which receive the major contributions are also shown for each background.

The topological distribution for the raw tau-candidate sample, the estimated backgrounds, and the background-subtracted sample is shown in Table 6.6 and Table 6.7 for the 1 prong tag and the 3 prong tag, respectively. In the next chapter, the unfold method is used to determine the most likely branching fractions of the 
Table 6.4. Backgrounds in the 1 prong tag. The firat column lists the source of the background and the second column lists the estimated total number of events from that source. The third column lists the major topological categories in which the background appears and the number of background events expected in that category.

\begin{tabular}{|c|c|c|}
\hline $\begin{array}{l}\text { Source } \\
e^{+} e^{-} \rightarrow\end{array}$ & $\begin{array}{c}\text { Total \# of background } \\
\text { events in } 207 \mathrm{pb}^{-1}\end{array}$ & $\begin{array}{c}\text { Major Topology, } \\
\text { \# of events }\end{array}$ \\
\hline$e^{+} e^{-}(\gamma)$ & $4.5 \pm 0.9$ & $(e, 0 \gamma), 3.9 \pm 0.8$ \\
\hline$\mu^{+} \mu^{-}(\gamma)$ & $0.5 \pm 0.2$ & $\begin{array}{l}(\mu, 0 \gamma), 0.2 \pm 0.1 \\
(\bar{c}, 0 \gamma), 0.3 \pm 0.1\end{array}$ \\
\hline$e^{+} e^{-} e^{+} e^{-}$ & $4.7 \pm 0.9$ & $(e, 0 \gamma), 3.8 \pm 0.7$ \\
\hline$e^{+} e^{-} \mu^{+} \mu^{-}$ & $4.4 \pm 1.6$ & $\begin{array}{l}(\mu, 0 \gamma), 2.3 \pm 0.8 \\
(z, 0 \gamma), 1.5 \pm 0.5\end{array}$ \\
\hline$g \bar{q}$ & $52 \pm 16$ & $\begin{array}{c}(\pi, \bar{c}, \bar{i} \geq 0 \gamma), 24 \pm 10 \\
(2 \text { or } 3 \text { prong, } 0 \gamma), 7 \pm 2 \\
(2 \text { or } 3 \text { prong, }>0 \gamma), 21 \pm 5\end{array}$ \\
\hline$e^{+} e^{-} \tau^{+} \tau^{-}$ & $35 \pm 5$ & $(c, 0 \gamma), 11 \pm 2$ \\
\hline
\end{tabular}

tau for the background-subtracted topologjcal distributions shown in these tables.
Table 6.5. Backgrounds in the 3 prong tag. The first column lists the source of the background and the second column lists the estimated total number of events from that source. The third column listo the majo topological categories in which the background appears and the number of background events expected in that category.

\begin{tabular}{|c|c|c|}
\hline $\begin{array}{c}\text { Source } \\
e^{+} e^{-} \rightarrow\end{array}$ & $\begin{array}{c}\text { Total \# of background } \\
\text { events in } 207 \mathrm{p}^{-1}\end{array}$ & $\begin{array}{c}\text { Major Topology, } \\
\text { \# of events }\end{array}$ \\
\hline$e^{+} e^{-}(\gamma)$ & 4 & $\begin{array}{c}(e, 0 \gamma), 3 \\
(\pi, 0 \gamma), 1\end{array}$ \\
\hline$\mu^{+} \mu^{-}(\gamma)$ & 0 & \\
\hline$e^{+} e^{-} e^{+} e^{-}$ & 0 & \\
\hline$e^{+} e^{-} \mu^{+} \mu^{-}$ & 0 & $(\pi, 2, \bar{\mu} ; \geq 0 \gamma), 10 \pm 2$ \\
\hline$q \bar{q}$ & $20 \pm 4$ & $(2$ or 3 prong, $0 \gamma), 3 \pm 1$ \\
& & $(2$ or 3 prong, $>0 \gamma), 7 \pm 2$ \\
\hline$e^{+} e^{-} \tau^{+} \tau^{-}$ & $15 \pm 3$ & $(e, 0 \gamma), 3 \pm 2$ \\
\hline
\end{tabular}


Table 6.6. Dintribution of raw tau-cendidate sample, background, and background oubtracted ample for the 1 prong tag.

\begin{tabular}{|c|c|c|c|c|c|}
\hline & Particle Identification & $\begin{array}{c}\text { Number } \\
\text { of Photons }\end{array}$ & Raw Sample & Background & $\begin{array}{l}\text { Background } \\
\text { Subtracted }\end{array}$ \\
\hline 1 & e & $\mathbf{0}$ & 261 & $19 \pm 3$ & 242 \\
\hline 2 & $\mu$ & n & 170 & $4 \pm 1$ & 166 \\
\hline 3 & $\pi$ & $\mathbf{0}$ & 169 & $2 \pm 1$ & 16.7 \\
\hline 4 & $\ddot{\varepsilon}$ & $\mathbf{0}$ & 120 & $4 \pm 2$ & 116 \\
\hline $\mathbf{5}$ & $\bar{\mu}$ & 0 & 30 & $2 \pm 1$ & 28 \\
\hline 6 & $x_{p}$ & & 128 & 1 & 127 \\
\hline 7 & $x_{d}$ & & 20 & 1 & 19 \\
\hline 8 & $\pi, z$ or $\bar{\mu}$ & 1 & 245 & $9 \pm 4$ & 236 \\
\hline 9 & $\pi, z$ or $\bar{\mu}$ & 2 & 141 & $6 \pm 2$ & 135 \\
\hline 10 & $\pi, 2$ or $\pi$ & 3 & 42 & $5 \pm 2$ & 37 \\
\hline 11 & $\pi, \bar{z}$ or $\bar{\mu}$ & 4 & 12 & $6 \pm 2$ & 6 \\
\hline 12 & $\pi, 2$ or $a$ & $>4$ & 0 & 0 & 0 \\
\hline 13 & e & $>0$ & 27 & $4 \pm 2$ & 23 \\
\hline 14 & $\mu$ & $>0$ & 7 & 0 & 7 \\
\hline 15 & 2 or 3 charged particles & 0 & 120 & $10 \pm 3$ & 110 \\
\hline 16 & 2 or 3 charged particles & $>0$ & 135 & $24 \pm 6$ & 111 \\
\hline & \multicolumn{2}{|c|}{ Total Number of Events } & 1627 & $97 \pm 13$ & 1530 \\
\hline
\end{tabular}

Table 6.7. Distribution of raw tau-candidate sample, background, and background subtracted sample for the 3 prong tag.

\begin{tabular}{|c|c|c|c|c|c|}
\hline & Particle Identification & $\begin{array}{l}\text { liumber } \\
\text { of Photons }\end{array}$ & Raw Sample & Background & $\begin{array}{l}\text { Background } \\
\text { Subtracted }\end{array}$ \\
\hline 1 & $e$ & 0 & 219 & $6 \pm 2$ & 213 \\
\hline 2 & $\mu$ & 0 & 109 & $\mathbf{0}$ & 109 \\
\hline 3 & $\pi$ & 0 & 78 & $5 \pm 2$ & 73 \\
\hline 4 & $\bar{c}$ & 0 & 189 & $2 \pm 2$ & 187 \\
\hline 5 & $\bar{\mu}$ & 0 & 19 & 0 & 19 \\
\hline 6 & $x_{p}$ & & 166 & $2 \pm 1$ & 164 \\
\hline 7 & $I_{d}$ & & 92 & $\mathbf{3} \pm \mathbf{1}$ & 89 \\
\hline 8 & $\pi, \bar{e}$ or $\bar{\mu}$ & 1 & 182 & $5 \pm 2$ & 177 \\
\hline 9 & $\pi, \bar{\epsilon}$ or $\bar{\mu}$ & 2 & 90 & $3 \pm 2$ & 93 \\
\hline 10 & $\pi, \bar{\varepsilon}$ or $\bar{\mu}$ & 3 & 49 & $2 \pm 1$ & 47 \\
\hline 11 & $\pi, z$ or $\bar{\mu}$ & 4 & 8 & $1 \pm 1$ & 7 \\
\hline 12 & $\pi, \bar{c}$ or $\bar{\mu}$ & $>4$ & 1 & 0 & 1 \\
\hline 13 & $e$ & $>0$ & 16 & 0 & 16 \\
\hline 14 & $\mu$ & $>0$ & 6 & 0 & 6 \\
\hline 15 & 2 or 3 charged particles & 0 & 102 & $4 \pm 1$ & 98 \\
\hline 16 & 2 or 3 charged particles & $>0$ & 143 & $7 \pm 2$ & 136 \\
\hline & \multicolumn{2}{|c|}{ Total Number of Events } & 1475 & $40 \pm 5$ & 1435 \\
\hline
\end{tabular}


7. Results

\subsection{Measured Branching fractions}

A maximum likelihood fit is used to estimate the branching fractions from the background subtracted distribution of tau-decay candidates corresponding to the 1 prong tag and the 3 prong tag. Because of the large uncertainties in the photon identification efficiency, and the amount of mixing of decay modes with two or more neutral pions in the final state among topologies with three or more detected photons, it is very difficult to determine branching fractions for $\tau^{-} \rightarrow \nu_{r} \pi^{-} 2 \pi^{0}$ and $r^{-} \rightarrow \nu_{\tau} \pi^{-3} 3 \pi^{0}$ separately, using this unethod. The results depend heavily on the minimum energy cutoff for a neutral energy cluster and the probability of finding opurious photons. Consequently, the three categories with a charged pion $(\pi, \bar{e}$ or $\bar{\mu})$ and three, four or more than four photons are combined into one category for the fit. Also, the decay modes $r^{-} \rightarrow \nu_{r} \pi^{-} 2 \pi^{0}$ and $\tau^{-} \rightarrow \nu_{r} \pi^{-} 3 \pi^{0}$ are combined assuming a nominal value of four for the ratio of $B\left(\tau^{-} \rightarrow \nu_{\tau} \pi^{-} 2 \pi^{0}\right)$ to $B\left(\tau^{-} \rightarrow \nu_{\tau} \pi^{-} 3 \pi^{0}\right)$ to calculate the corresponding row in the efficiency matix. This ratio is varied to estimate the systernatic errors due to the uncertainty in the ratio.

The observed distribution of tau-decay candidates, and the distribution corresponding to the branching fractions which result in a maximum of the likelihood function, are shown in Table 7.1 for the samples corresponding to each tag. The measured branching fractions are shown in Table 7.2. The errora correspond to a half unit change in the log likelihood, as described in Section 5.3. The correlation coefficients for the fit are shown in Table 7.3 and Table 7.4 for the 1 prong tag and the 3 prong tag, respectively. Note that the correlation coefficients between the leptonic modes and the semileptonic modes are generally quite small. An exception is the correlation between the leptonic modes and the mode $r^{-} \rightarrow 2 \cdot \pi^{-}$, especially for the sample corfesponding to the 3 prong tag. The highest correlation exists between $\tau^{-} \rightarrow \nu_{r} \pi^{-} \pi^{0}$ and $\tau^{-} \rightarrow \nu_{\tau} \pi^{-}\left(2\right.$ or $\left.3 \pi^{0}\right)$.

Table 7.1. Topological distribution of the background subtracted sample and the distribution corresponding to the best fit for the 1 prong tag and the 3 prong tag.

\begin{tabular}{|c|c|c|c|c|c|}
\hline \multirow{2}{*}{$\begin{array}{c}\text { Particle } \\
\text { Identification }\end{array}$} & \# of & \multicolumn{2}{|c|}{ 1 prong tag } & \multicolumn{2}{c|}{ 3 prong tag } \\
\cline { 3 - 6 } & photons & observed & best fit & observed & best fit \\
\hline$\mu$ & 0 & 242 & 243 & 213 & 211 \\
$\pi$ & 0 & 166 & 159 & 109 & 103 \\
$\bar{e}$ & 0 & 167 & 164 & 73 & 73 \\
$\bar{\mu}$ & 0 & 116 & 126 & 187 & 190 \\
$x_{p}$ & 0 & 28 & 23 & 19 & 15 \\
$x_{\mathrm{d}}$ & & 127 & 128 & 164 & 166 \\
$\pi, \bar{e}$ or $\bar{\mu}$ & 1 & 236 & 242 & 177 & 163 \\
$\pi, \bar{e}$ or $\bar{\mu}$ & 2 & 135 & 124 & 93 & 105 \\
$\pi, \bar{e}$ or $\bar{\mu}$ & $>2$ & 43 & 48 & 55 & 50 \\
$e$ & $>0$ & 27 & 21 & 16 & 18 \\
$\mu$ & $>0$ & 7 & 6 & 6 & 6 \\
\multirow{2}{*}{3 or 3 charged particles } & 0 & 110 & 110 & 98 & 98 \\
2 or 3 charged particles & $>0$ & 111 & 111 & 136 & 136 \\
\hline Total Number of Events & 1530 & 1530 & 1435 & 1435 \\
\hline
\end{tabular}


Table 7.2. Measured branching fractions (in per cent). The error for each measurement is the statistical error only.

\begin{tabular}{|c|c|c|}
\hline $\begin{array}{c}\text { Decay Mode } \\
r^{-} \rightarrow\end{array}$ & 1 Prong Tag & 3 Prong Tag \\
\hline$e^{-} D_{e} \nu_{r}$ & $19.3 \pm 1.2$ & $18.7 \pm 1.2$ \\
$\mu^{-} \nu_{\mu} \nu_{r}$ & $17.4 \pm 1.2$ & $19.1 \pm 1.4$ \\
$\pi^{-} \nu_{r}$ & $11.3 \pm 1.4$ & $7.7 \pm 1.7$ \\
$\pi^{-} \pi^{0} \nu_{\tau}$ & $25.0 \pm 2.6$ & $26.2 \pm 2.3$ \\
$\pi^{-}\left(2\right.$ or $\left.3 \pi^{0}\right) \nu_{r}$ & $14.0 \pm 2.2$ & $10.6 \pm 1.8$ \\
$\pi^{-} \pi^{+} \pi^{-} \nu_{r}$ & $6.8 \pm 1.1$ & $7.3 \pm 1.1$ \\
$\pi^{-} \pi^{+} \pi^{-} \pi^{0} \nu_{r}$ & $4.2 \pm 1.2$ & $8.4 \pm 1.2$ \\
\hline$K^{-} \nu_{\tau}$ & \multicolumn{2}{|c|}{ fixed at $0.7 \%$} \\
$(K \pi)^{-} \nu_{r}$ & \multicolumn{2}{|c|}{ fixed at $1.3 \%$} \\
\hline
\end{tabular}

Table 7.3. Correlation coefficients for the measurements corresponding to the 1 prong tag.

\begin{tabular}{|c|c|c|c|c|c|c|c|}
\hline Decay Mode & $e^{-} \nu_{e}$ & $\mu^{-} \nu_{\mu}$ & $\pi^{-}$ & $\pi^{-} \pi^{0}$ & $\pi^{-}\left(2\right.$ or $\left.3 \pi^{0}\right)$ & $3 \pi^{ \pm}$ & $3 \pi^{ \pm} \pi^{0}$ \\
\hline$e^{-} \nu_{e}$ & 1 & & & & & & \\
\hline$\mu^{-} \nu_{\mu}$ & -0.175 & 1 & & & & & \\
\hline$\pi^{-}$ & -0.248 & -0.318 & 1 & & & & \\
\hline$\pi^{-} \pi^{0}$ & -0.118 & -0.079 & -0.416 & 1 & & & \\
\hline$\pi^{-}\left(2\right.$ or $\left.3 \pi^{0}\right)$ & -0.112 & -0.097 & 0.133 & -0.760 & 1 & & \\
\hline $3 \pi^{ \pm}$ & -0.080 & -0.051 & -0.015 & -0.126 & 0.071 & 1 & \\
\hline $3 \pi^{ \pm} \pi^{0}$ & -0.019 & -0.034 & -0.053 & 0.112 & -0.288 & -0.626 & 1 \\
\hline
\end{tabular}

7.2 SYSTEMATIC ERRORS

Systematic errors are introduced by uncertainties in particle identification
Table 7.4. Correlation coefficients for the measurements corresponding to the 3 prong tag.

\begin{tabular}{|c|c|c|c|c|c|c|c|}
\hline Decay Mode & $e^{-} \nu_{e}$ & $\mu^{-} \nu_{\mu}$ & $\pi^{-}$ & $\pi^{-} \pi^{0}$ & $\pi^{-}\left(2\right.$ or $\left.3 \pi^{0}\right)$ & $3 \pi^{ \pm}$ & $3 \pi^{ \pm} \pi^{0}$ \\
\hline$e^{-} \nu_{e}$ & 1 & & & & & & \\
\hline$\mu^{-} \Sigma_{\mu}$ & -0.119 & 1 & & & & & \\
\hline$\pi^{-}$ & -0.373 & -0.472 & 1 & & & & \\
\hline$\pi^{-} \pi^{0}$ & -0.060 & -0.082 & -0.375 & 1 & & & \\
\hline$\pi^{-}\left(2 \circ{ }^{0} 3 \pi^{0}\right)$ & -0.206 & -0.068 & 0.073 & -0.658 & 1 & & \\
\hline $3 \pi^{ \pm}$ & -0.169 & 0.114 & -0.112 & -0.258 & 0.017 & 1 & \\
\hline $3 \pi^{ \pm} \pi^{0}$ & -0.047 & -0.330 & 0.055 & 0.119 & -0.296 & -0.556 & 1 \\
\hline
\end{tabular}

efficiencies, relative overall efficiencies for each decay mode, and background estimates. The contribution of each of these sources to the systematic error is discussed in the following sections and the systematic errors are summarized in Table 7.5.

\subsubsection{Particle Identification Efficiency}

The uncertainty in the election identification efficiency is estimated to be $3 \%$. It is small because of the large sample of electrons, with little background, available in the data for checking the efficiency. The uncertainty in pion identification efficiency is slightly higher since clean samples of isolated charged pions, with a known number of neutral pions accompanying them, are difficult to find in the data. The uncertainty in the pion identification efficiency is estimated to be $4 \%$. Variations in the branching fractions are estimated for changes in the electron and pion identification efficiencies equal to the uncertainty. 
In addition, the analysis is repeated using different cutoff values of TEST 1 for $e / \pi$ discrimination. A low cutoff value of 0.65 , resulting in a clean pion sample, and a high eutof value of 1.10 , resulting in a clean electron sample, are uged. The dependence of the results on the cutoff value of TEST1 used in the analysis is negiigible.

The uncertainty in the muon identification efficiency is estimated to be $3 \%$. Again, this uncertainty is small because of the clean sample of muons available for checking the efficiency. The uncertainty in the probability for a pion to pass the identification criteria for a muon is estimated to be $30 \%$. The effect of this uncertainty on the results is negligible.

The uncertainty in the number of excess fake photons in the data is estimated to be $50 \%$; i.e. the number of fake photons in the data is higher than the number predicted by the Monte Carlo by $(50 \pm 25) \%$.

The sensitivity to photon identification is also estimated by varying the cutoff used tor the minimum energy of a neutral energy cluster. The results for $B\left(\tau^{-} \rightarrow \nu_{\tau} \pi^{-} \pi^{0}\right)$ and $B\left(\tau^{-} \rightarrow \nu_{\tau} \pi^{+}\left(2\right.\right.$ or $\left.\left.3 \pi^{0}\right)\right)$ are quile sensitive to this cutoff.

A low cutoff increases the number of spurious fake photons found, while a high cutoff eliminates real photons. Both effects cause a loss of sensitivity resulting in a fit which is not robust. This sensitivity is included in the systematic error due to photon identification.

Recall that the decay modes $\tau^{-} \rightarrow \nu_{T} \pi^{-} 2 \pi^{0}$ and $r^{-} \rightarrow \nu_{T} \pi^{-} 3 \pi^{0}$ are combined assuming a nominal value of four for the ratio of $B\left(r^{-} \rightarrow \nu_{r} \pi^{-} 2 \pi^{0}\right)$ to $B\left(r^{-} \rightarrow\right.$ $\nu_{r} \pi^{-3} \pi^{0}$ ) to calculate the carresponding row in the efficiency matix. The value of this ratio is varied between two and eight to estimate the systematic errors due to the uncertainty in the ratio. The errors introduced due to the uncertainty in this ratio are small compared to other systematic errors.

\subsubsection{Relative Overall Efficiency}

The uncertainty in the branching fractions due to the bias in the sample is directly proportional to the uncertainty in the relative overall efficiency for each decay mode. These efficiencies, determined from the Monte Carlo, are listed in Table 5.3 and 5.4 for the 1 prong tag and the 3 prong tag, respectively. The errors listed in these tables are statistical errors only and represent an uncertainty of about $3 \%$ for the major decay modes. Within each sample, there is little or no dependente of the relative efficiency on the decay mode of the tag, according to the Monte Carlo. For example, in the 1-prong-tag sample, the relative efficiencies do not depend on whether the tag corresponds to $\tau^{-} \rightarrow \nu_{\mathrm{T}} \pi^{-}$or $\tau^{-} \rightarrow \nu_{T} \rho^{-}$.

The Monte Carlo does not predict any significant differences in efficiency for different decay modes for the sample corresponding to the 1 prong tag. However, it does predict that the electron and muan decay modes are more likely to be in iuded in the sample corresponding to the 3 prong tag.

The uncertainty in the relative overall efficiency for each decay mode is estimated to contribute an absolute error of $0.8 \%$ to each branching fraction. For the electron and muon decay modes, this corresponds to a relative error of about $4.5 \%$. 


\subsubsection{Background Estimates}

The errors due to the uncertainty in the backgrounds are estimated by varying the backgrounds within the ranges shown in Tables 6.6 and 6.7. The background from each independent source is varied separately. The errots from the uncertainties in background are generally small compared to the overall systematic errors.

\subsubsection{Summary of Systematic Errors}

A summary of the absolute systematic errors for each branching fraction is shown in Table 7.5. The major contributions to the systematic errors for the leptonic decay modes are the uncertainties in the relative overall efficiencies and the particle identification efficiencies. For the hadronic modes, the major contribution to the error is the uncertainty in photon identification. For all the decay modes, the total systematic error is approximately the same tize as the statistical error from the maximum likelihood fit.

7.3 RESULTS

The measured branching fractions for the tau candidate samples corresponding to the 1 prong tag and the 3 prong tag are shown in Table 7.6. For each branching fraction, the frst error is statistical and the second is systematic. The statistically weighted average of the measurements corresponding to the two tags is also shown for each branching fraction. The systematic error for the weighted average is obtained by averaging systematic errors common to the two samples linearly (for example, particle identification efficiency), and other systematic errors quad ratically.
Table 7.5. Absolute zystematic errore (in per cent) for the measurement corresponding to the 1 prong tag and the 3 prong tag.

\begin{tabular}{|c|c|c|c|}
\hline $\begin{array}{c}\text { Decay Mode } \\
\tau^{-} \rightarrow\end{array}$ & $\begin{array}{l}\text { Source of Error: } \\
\text { Uncertainty in }\end{array}$ & $\begin{array}{c}1 \text { Prong Tag } \\
\text { Error (\%) }\end{array}$ & $\begin{array}{l}3 \text { Prong Tag } \\
\text { Error(\%) }\end{array}$ \\
\hline All Modes & Relative Overall Efficiencies & 0.8 & 0.8 \\
\hline$e^{-} D_{e} V_{\mathrm{r}}$ & $\begin{array}{c}e / \pi \text { Discrimination for } e^{ \pm} \\
e / \pi \text { Discrimination for } \pi^{ \pm} \\
\text {Backgrounds } \\
\text { Total }\end{array}$ & $\begin{array}{l}0.6 \\
0.7 \\
0.4 \\
1.3\end{array}$ & $\begin{array}{l}0.6 \\
0.5 \\
0.3 \\
1.2\end{array}$ \\
\hline$\mu-\nu_{\mu} \nu_{\tau}$ & $\begin{array}{c}\mu / \times \text { Discrimination for } \mu^{ \pm} \\
\text {Backgrounds } \\
\text { Total }\end{array}$ & $\begin{array}{l}0.5 \\
0.3 \\
1.0\end{array}$ & $\begin{array}{l}0.5 \\
0.2 \\
1.0\end{array}$ \\
\hline$\pi-\nu_{r}$ & $\begin{array}{c}e / \pi \text { Discrimination for } e^{ \pm} \\
e / \pi \text { Discrimination for } \pi^{ \pm} \\
\mu / \pi \text { Disrimination for } \mu^{ \pm} \\
\text {Photon Identification } \\
\text { Backgrounds } \\
\text { Total }\end{array}$ & $\begin{array}{l}0.5 \\
0.6 \\
0.6 \\
0.8 \\
0.2 \\
1.5\end{array}$ & $\begin{array}{l}0.6 \\
0.4 \\
0.6 \\
0.8 \\
0.2 \\
1.5\end{array}$ \\
\hline$\pi^{-} \pi^{0} \nu_{\tau}$ & $\begin{array}{c}\text { e/ Discrimination for } \pi^{ \pm} \\
\text {Ratio of } B\left(\pi^{-}-2 \pi^{0}\right) \text { to } B\left(\pi^{-}-3 \pi^{0}\right) \\
\text { Photon Identification } \\
\text { Backgrounds } \\
\text { Total } \\
\end{array}$ & $\begin{array}{l}1.2 \\
1.0 \\
2.0 \\
0.3 \\
2.7\end{array}$ & $\begin{array}{l}1.1 \\
0.4 \\
2.0 \\
0.2 \\
2.5\end{array}$ \\
\hline$\pi^{-}\left(2\right.$ or $\left.3 \pi^{0}\right) \nu_{\tau}$ & $\begin{array}{c}\text { e/ } / \pi \text { Discrimination for } \pi^{ \pm} \\
\text {Ratio of } B\left(\pi^{-}-2 \pi^{0}\right) \text { to } B\left(\pi^{-}-3 \pi^{0}\right) \\
\text { Photon Identification } \\
\text { Backgrounds } \\
\text { Total }\end{array}$ & $\begin{array}{l}1.2 \\
1.0 \\
2.0 \% \\
0.8 \\
2.8\end{array}$ & $\begin{array}{l}1.1 \\
0.4 \\
2.0 \\
0.2 \\
2.5 \\
\end{array}$ \\
\hline$\pi^{-} \pi^{+} \pi^{-} L^{\prime} \tau$ & $\begin{array}{c}\text { Photon Identification } \\
\text { Backgrounds } \\
\text { Total } \\
\end{array}$ & $\begin{array}{l}0.6 \\
0.7 \\
1.2 \\
\end{array}$ & $\begin{array}{l}0.6 \\
0.3 \\
1.0 \\
\end{array}$ \\
\hline$\pi^{-} \pi^{+} \pi^{-} \pi^{0} \nu_{r}$ & $\begin{array}{c}\text { Photon Identification } \\
\text { Backgrounds } \\
\text { Total }\end{array}$ & $\begin{array}{l}0.6 \\
0.7 \\
1.2\end{array}$ & $\begin{array}{l}0.6 \\
0.3 \\
1.0\end{array}$ \\
\hline
\end{tabular}


Table 7.6. Measured branching fractions (in per cent). For each measurement, the first error is statistical and the second is systematic.

\begin{tabular}{|c|c|c|c|}
\hline $\begin{array}{c}\text { Decay Mode } \\
\tau^{-} \rightarrow\end{array}$ & 1 Prong Tag & 3 Prong Tag & Average \\
\hline$e^{-} \nu_{e} \nu_{\tau}$ & $19.3 \pm 1.2 \pm 1.3$ & $18.7 \pm 1.2 \pm 1.2$ & $19.0 \pm 0.8 \pm 1.1$ \\
$\mu^{-} \nu_{\mu} \nu_{\tau}$ & $17.4 \pm 1.2 \pm 1.0$ & $19.1 \pm 1.4 \pm 1.0$ & $18.1 \pm 0.9 \pm 0.8$ \\
$\pi^{-} \nu_{\tau}$ & $11.3 \pm 1.4 \pm 1.5$ & $7.7 \pm 1.7 \pm 1.5$ & $9.9 \pm 1.1 \pm 1.4$ \\
$\pi^{-} \pi^{0} \nu_{r}$ & $25.0 \pm 2.6 \pm 2.7$ & $26.2 \pm 2.3 \pm 2.5$ & $25.6 \pm 1.7 \pm 2.5$ \\
$\pi^{-} 2$ or $3 \pi^{0} \nu_{\tau}$ & $14.0 \pm 2.2 \pm 2.8$ & $10.6 \pm 1.8 \pm 2.5$ & $12.0 \pm 1.4 \pm 2.5$ \\
$\pi^{-} \pi^{+} \pi^{-} \nu_{\tau}$ & $6.8 \pm 1.1 \pm 1.2$ & $7.3 \pm 1.1 \pm 1.0$ & $7.1 \pm 0.8 \pm 0.9$ \\
$\pi^{-} \pi^{+} \pi^{-} \pi^{0} \nu_{\tau}$ & $4.2 \pm 1.2 \pm 1.2$ & $8.4 \pm 1.2 \pm 1.0$ & $6.3 \pm 0.8 \pm 0.9$ \\
\hline$K^{-} \nu_{\tau}$ & & fixed at $0.7 \%$ & \\
$(K \pi)^{-} \nu_{r}$ & & fixed at $1.3 \%$ & \\
\hline
\end{tabular}

In this analysis, the sum of the branching fractions is constrajned to one. Since the decay modes used in the fit aje only those for which the branching fractions have been measured in other experiments, and since the sum of the previously measured branching fractions is less than one, this analysis must result in a larger branching fraction than the world average for at least one mode.

The following comments pertain to the results.

- The total branching fraction to three charged particles is measured to be $(13.4 \pm 1.1 \pm 0.7) \%$ which is in good agreement with the world average of $B 3=$ (13.1 \pm 0.3$) \%$. The ratio of $B\left(\tau^{-} \rightarrow \nu_{r} \pi^{-} \pi^{+} \pi^{-}\right)$to $B\left(\tau^{-} \rightarrow \nu_{r} \pi^{-} \pi^{+} \pi^{-} \pi^{0}\right)$ is also in agreement with the world average.

- a arh tag, both leptonic branching fractions are found to be higher than those measured in previous experiments. The measured branching fraction for $r^{\prime} \rightarrow \nu_{r} e^{-} \nu_{e}$ of $(19.0 \pm 0.8 \pm 1.1) \%$ is higher than the world average of $(17.9 \pm 0.4) \%$ by $(1.1 \pm 1.4) \%$. The meaured branching fraction for $r^{-} \rightarrow \nu_{\tau} \mu^{-} \nu_{\mu}$ of $(18.1 \pm 0.9 \pm 0.8) \%$ is higher than the world average of $(17.2 \pm 0.4) \%$ by $(0.9 \pm 1.2) \%$. The total excess in the leptonic branching fractions is $(2.0 \pm 1.8) \%$. The leptonic branching fractions from this analysis correspond to a tau lifetime of $(3.0 \pm 0.2) \times 10^{-13} \mathrm{~s}$. Recall that the average measured tau lifetime is $(2.8 \pm 0.2) \times 10^{-13} s$. Therefore, the leptonic branching fractions measured in this analysis are consistent with the measured tau lifetime.

- The theoretical prediction for the ratio of $B\left(r^{-} \rightarrow \nu_{r} \pi^{-}\right)$to $B\left(r^{-} \rightarrow \nu_{r} e^{-} D_{e}\right)$ is 0.607 . Combining this with the measured leptonic branching fractions from this analysis leads to a prediction of $B\left(\tau^{-} \rightarrow \nu_{+} \pi^{-}\right)=(11.4 \pm 0.6) \%$ which is $(1.5 \pm 1.9) \%$ higher than the measured value of $(9.9 \pm 1.1 \pm 1.4) \%$.

- A significant difference between the measured branching fractions and theoretical expectations involves the decays to one charged hadion and multiple neutrals. The measurements of the branching fractions for the decay modes $\tau^{-} \rightarrow \nu_{\tau} \pi^{-} \pi^{0}$ and $\tau^{-} \rightarrow \nu_{*} \pi^{-}\left(2\right.$ or $\left.3 \pi^{0}\right)$ have large errors which are highly correlated. The difference between unity and the sum of the branching fractions for all the other decay modes (which are not as highly correlated) is measured to be $(37.6 \pm 2.9) \%$. The theoretical prediction for the sum of the branching fractions for $\tau^{-} \rightarrow \nu_{\mathrm{r}} \rho^{-}, \tau^{-} \rightarrow \nu_{\mathrm{r}} \pi^{-} 2 \pi^{0}$, and 
$\tau^{-} \rightarrow \nu_{r} \pi^{-} 3 \pi^{0}$ is about $31 \%$ (see Table 1.14). Therefore, the measured value for $B\left(\tau^{-} \rightarrow \nu_{\tau} \pi^{-}\left(n \pi^{0}\right), n>0\right)$ is larger than predicted by theory by about $(7 \pm 3) \%$. This difference indicates a larger branching fraction to one charged pion plus multipie neutral pions than expected from theoretical predictions, or the presence of other decay modes, such as those with an $\eta$ in the final state.

\subsubsection{Limits on Decay Modes Involving the $\eta$}

Recall fron the discussion in section 1.3.3 that $B\left(r^{-} \rightarrow \nu_{r} \pi^{-} \eta \pi^{0}\right)$ is at most $0.7 \%$ based on the assumption that the final state is produced via the $\rho(1600)$ resonance and on measured branching fractions for the $\rho(1600)$. Limits can also be set on the other two possible decay modes involving $\eta$ 's based on the measured branching fraction for the decay of the tau to ive charged particles. The efficiency for reconstructing these modes as five charged particles is compared to the efficiency for detecting $\mathrm{I}^{-} \rightarrow \nu, 5 \pi^{ \pm} \pi^{0}$ with the final state distributed purely according to phase space which is the efficiency used in the Mark II measurement of B5. ${ }^{5}$ The efficiency for detecting the five prong mode of $r^{-} \rightarrow \nu_{\tau} \pi^{-} \eta \pi^{+} \pi^{-}$is the same as for $\tau^{-} \rightarrow \nu_{\tau} 5 \pi^{ \pm} \pi^{0}$ but the efficiency for detecting the five prong mode of $\tau^{-} \rightarrow \nu_{\tau} \pi^{-} \eta \eta$ is only about half of the efficiency for $\tau^{-} \rightarrow \nu_{\tau} 5 \pi^{ \pm} \pi^{0}$. Therefore, the measured value of B5 can be used to set the following limits:

$$
\begin{gathered}
B\left(\tau^{-} \rightarrow \nu_{q} \pi^{-} \eta \pi^{+} \pi^{-}\right) \leq 0.5 \%, \\
B\left(\tau^{-} \rightarrow \nu_{q} \pi^{-} \eta \eta\right) \leq 3 \% .
\end{gathered}
$$

Recall that $B\left(\tau^{-} \rightarrow \nu_{+} \pi^{-} \eta \pi^{0} \pi^{0}\right) \leq B\left(\tau^{-} \rightarrow \nu_{\tau} \pi^{-} \eta \pi^{+} \pi^{-}\right)$by isospin conservation.

Therefore, the total contribution of decay modes involving the $\eta$ to the branching fraction for final states containing one charged pion and multiple neutrals is expected to be less than $2.4 \%$.

\subsection{CONCLUSIONS}

The results of this analysis indicate that the discrepancy between the inclusive branching fraction and the surn of the exclusive branching fractions to final states containing one charged particle found in previous experiments is possibly due to two sources.

First, the leptonic branching fractions measured in this analysis are about one standard deviation higher than the world average, resulting in a total excess of $(2.0 \pm 1.8) \%$ over the world average in these two modes. The measured leptonic branching fractions correspond to a tau lifetime of $(3.0 \pm 0.2) \times 10^{-13} \mathrm{~s}$ which is slightly longer than the average measured tau lifetime of $(2.8 \pm 0.2) \times 10^{-13} \mathrm{~s}$. This longer lifetime reduces the discrepancy predicted by theoretical calculations from about $9 \%$ to $2 \%$.

The total branching fraction to one charged hadron plus at least une neutral particle is measured to be $(7 \pm 3) \%$ higher than the branching fraction expected from a combination of previous measurements and theoretical predictions. Decay modes involving the $\eta$ are nor expected to contribute more than about $3 \%$ to this excess. 
Appendix A. Selection of Known Leptons in the Data

The lepton identification efficiency is estimated using two sources of known - leptons in the data: single photon production of $e^{+} e^{-}$pairs with initial or final state radiation, and $\mu^{+} \mu^{-}$ọirs with or without initial or final state radiation; and two photon production of $e^{+} e^{-}$and $\mu^{+} \mu^{-}$pairs.

The radiative lepton pairs are chosen to provide leptons with a momentum spectrum which extends below $14.5 \mathrm{GeV} / \mathrm{c}$. The sample is obtained by selecting events with two charged tracks and ore neutral energy cluster with at least $1 \mathrm{GeV}$ of energy separated from the charged tracks by at least $20 \mathrm{~cm}$ in the $L A$ calorimeter. For the electron sample, the two charged tracks project into different LA calorimeter modules. The sum of the momenta of the two charged tracks, as measured by the drift chamber, and the energy of the neut=al energy cluster is at least $24 \mathrm{GeV}$.

The sample of radiative $e^{t} e^{-}$pairs contains an ample rumber of electron candidates, However, the muon candidate sample does not have a large number of events. To improve the statistics at high momentum, the muon candidate sample is supplemented with nonradiati:.. tribun pairs. This sample is obtained by selecting events with two charged tracks and no nelitral energy clusters of more

、 than $200 \mathrm{MeV}$. The sum of the momenta of the two charged tracks is greater than $22 \mathrm{GeV} / \mathrm{c}$ and less than $36 \mathrm{GeV} / \mathrm{c}$. The time-of-flight information is used to reject cosmic ray events.

The sample of two photon produced events is obtained by selecting events with two charged tracks and no neutral energy clusters of more than $200 \mathrm{MeV}$.
The oum of the momenta of the two charged tracks is less than $12 \mathrm{GeV} / c$ and the net momentum in the event trangverse to the beam is less than $300 \mathrm{MeV} / \mathrm{c}$. The time-of-fight information is used to reject cosmic ray events.

For all of the above samples, each charged track is tested with strict leptos identification criteria. For the nonradiative muon pajr sample, the momentum of this track must be between 12 and $16 \mathrm{GeV} / \mathrm{c}$. If the track passes the criteria for an electron (or muon), the other charged track in the event is included in the sample used to check the electron (or muon) rejection efficiency. This results in an unbiassed sample of electrons and muons with a small contamination from $r$-pair production.

The momentum distribution for each sample is shown in Figure A.1 for electrons and muons, separately. The total number of electron candidates is 15555 and the total number of muon candidites is $\mathbf{4} 923$. The number of muons is smaller than the number of electrons for the following reasons The cross section for single photon production of $e^{+} e^{-}$pairs is much larger than the cross section for single photon production of $\mu^{-t} \mu^{-}$pairs. Also, the radiative muon sample is much smaller than the radiativ electron sample because of the large mass difference. Finally, the solid angle coverage of the muon system is smaller than that of the LA calorineter, and the minimum momentum cutoff for muon identification is $2 G \mathrm{eV} / \mathrm{c}$ while it is $1 \mathrm{GeV} / \mathrm{c}$ for electron identification. 

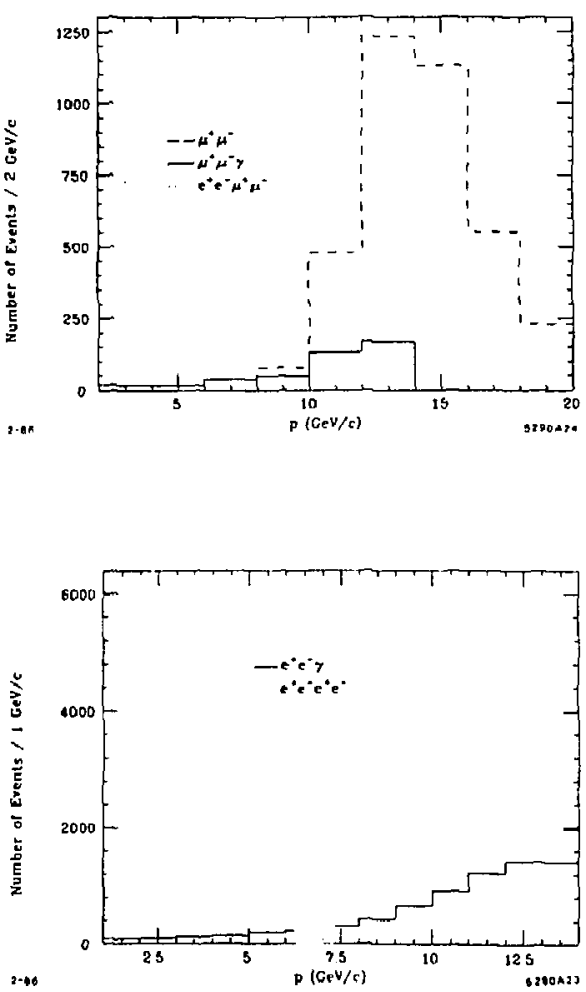

Figure A.1. Momentum distributions for known muons and electrons in the data. The solid histogram corresponds to single photon production of lepton pairs with initial or final state radiation. The dashed histogram corresponds to single photon production of muon pairs without initial or final state radiation and the dotied histogram corresponds to two photon production of lepton pairs.

\section{REFERENCES}

1. M. L. Perl et al., Phys. Rev. Lett. 35, 1489 (1975)

2. M. L. Perl et al., Phys. Lett, B 63, 466 (1976)

3. M. L. Perl et al., Phys. Lett. B 70, 487 (1977)

4. C. A. Blocker et al., Phys. Rev. Lett. 40, 1369 (1982)

5. P. R. Burchat et al., Phys. Rev. Lett. 54, 2489 (1985)

6. H. J. Behrend et al., Phys. Lett. B 114, 282 (1982)

7. H. J. Behrend et al., Z. Physik C 23, 103 (1984)

8. Ch. Berger et al., Z. Physik G 28, 1 (1985)

9. M. Althoff et al., Z. Physik C 26, 521 (1985)

10. H. Aihara et al., Phys. Rev. D 30, 2436 (1984)

11. E. Fernandez et al., Phys. Rev. Lett. 54, 1624 (1985)

12. M. Derrick et al., Phys. Rev. Lett. 55, 570 (1985)

13. M. Derrick et al, Phys, Rev, Lett. 54, 1775 (1985)

14. W. Bartel et al., Phys. Lett. B 101, 188 (1985)

15. W. Ruckstuhl et al., submitted to Phys. Rev. Lett. (1986)

16. Y. S. Tsai, Phys. Rev. $D$ \&, 2821 (1971)

17. F. J. Gilman and Sun Hong Rhie, Phys. Rev. D 31, 1066 (1985)

18. G. J. Feldman et al, Phys. Rev, Lett. 48, 66 (1982)

19. H. J. Behrend at al., Nucl. Phys. B 211, 369 (1983)

20. J. Jaros, SLAC-PUB-3569, Proceedings of the 12th SLAC Summer Institute on Particle Physics, ed. by P. McDonough, p. 427 (1984) 
21. M. Althof et al., Phys. Lett. B 141, 264 (1984)

22. MAC collaboration, in Proceedings of the International Symposium on Lepton and Photon Interactions at High Energies, Kyoto, Japan (1985) (to be published)

23. HRS collaboration, in Proceedings of the International Symposium on Lepton and Photon Interactions at High Energies, Kyoto, Japan (1985) (to be published)

24. C. A. Blocker et al., Phys. Lett. B 109, 119 (1982)

25. H. J. Behrend et al., Phys. Lett. B 127, 270 (1983)

26. R. M. Baltrusaitis et al., Phys. Rev. Lett. 55, 1842 (1985)

27. W. W. Ash et al., Phys. Rev. Lett. 55, 2118 (1985)

28. G. S. Abrams et al., Phys. Rev. Lett. 43, 1555 (1979)

29. J. M. Yelton et al., SLAC-PUB-3579, submitted to Phys. Rev. Lett. (1985)

30. W. B. Schmidke et al, private communication (1985)

31. C. A. Blocker et al., Phys. Rev. Lett. 48, 1586 (1982)

32. G. B. Mills et al., Phys. Rev. Lett. 52, 1944 (1984)

33. H. Aihara et al, Phys. Rev. D 30, 2436 (1984)

34. J. M. Dorfan et al., Phys. Rev. Lett. 46, 215 (1982)

35. Particle Data Book, Rev. Mod. Phys. 56 (1984)

36. G. B. Mills et al., Phys. Rev. Lett. 54, 624 (1985)

37. K. G. Hayes et al., Phys. Rev. D 25, 2869 (1982)

38. M. E. Levi et al., Phys. Rev, Lett. 51, 1941 (1983)
39. K. K. Gan et al., Phys. Lett. B 153, 116 (1985)

40. W. B. Schmidke, private communication (1985)

41. F. James and M. Roos, Comp. Phys. Comm. 10, 343 (1975)

42. F. A. Berends and R. Kleiss, Nucl. Phys. B 177, 239 (1981)

43. R. L. Ford and W, R. Nelson, SLAC-PUB-2107 (1978)

44. S. J. Brodsky, T. Kinoshita and H. Terazawa, Phys. Rev. D 4,1532 (1971) 